\title{
22. PALEOENVIRONMENTAL RESULTS FROM NORTH ATLANTIC SITES 607 AND 6091
}

\author{
William F. Ruddiman, Lamont-Doherty Geological Observatory \\ Andrew McIntyre, Lamont-Doherty Geological Observatory, and Queens College of the City \\ University of New York \\ and \\ Maureen Raymo, Lamont-Doherty Geological Observatory ${ }^{2}$
}

\begin{abstract}
Detailed $\mathrm{CaCO}_{3}$ analyses from Leg 94 Sites 607 and 609 confirm earlier findings from Leg 81 that large-scale Northern Hemisphere glaciation began near the Gauss/Matuyama boundary. At Site 609, the first ice-rafted sand occurs between samples at 2.55 and $2.45 \mathrm{Ma}$, or effectively coincident with the Gauss/Matuyama paleomagnetic boundary. Although small to moderate decreases in the $\mathrm{CaCO}_{3}$ percentage from maxima in the lower Pliocene began at $3.15 \mathrm{Ma}$, the lack of ice-rafted debris until $2.55 \mathrm{Ma}$ suggests that this decline must be explained by other factors, particularly dissolution. The records at Sites 607 and 609 show an increase in the amplitude of $\mathrm{CaCO}_{3}$ variations in the last million years.

Counts of planktonic foraminifers reveal complex changes in estimated sea-surface temperature (SST) through the last 1.90 m.y., including long-term trends with wavelengths of several hundred thousand years and rhythmic changes with wavelengths of several tens of thousands of years. Relatively warm conditions prevailed until $1.05 \mathrm{Ma}$, and the lowest SST values of the Pleistocene in this area occurred near 0.8 and $0.7 \mathrm{Ma}$. In several instances, unusually low glacial temperatures were immediately followed by unusually high temperatures in the succeeding interglaciation.
\end{abstract}

\section{INTRODUCTION}

Leg 94 was designed primarily as a north-south transect for paleoenvironmental study of the North Atlantic from the northern part of the modern subtropical gyre to the east-central portion of the subpolar gyre. We have focused initial efforts in this study on two of the six sites cored: 607 and 609 (Fig. 1). Analysis of upper Quaternary piston cores at the locations of these two sites has shown a strong development of the basic orbital rhythms at $23,000,41,000$, and 100,000 yr. in records of North Atlantic sea-surface temperature (SST) change (Ruddiman and McIntyre, 1984). We thus chose these two sites to trace the rhythms of sea-surface change into earlier periods, as well as to examine the long-term history of $\mathrm{CaCO}_{3}$ deposition in the North Atlantic, and the inferred glacial history on the surrounding continents.

We report detailed 3.5-m.y. records of $\mathrm{CaCO}_{3}$ from both sites, as well as a 1.90-m.y. record of oxygen and carbon isotopic values, planktonic foraminiferal counts, and estimated sea-surface temperatures from Site 607 .

\section{$\mathrm{CaCO}_{3}$ VARIATIONS AT SITES 607 AND 609 (3.5-0 Ma)}

$\mathrm{CaCO}_{3}$ analyses of Site 609 samples were carried out at $30-\mathrm{cm}$ intervals, equivalent to a mean sample interval of about 4000 yr. Analyses of Site 607 samples were performed at $15-\mathrm{cm}$ intervals, equivalent to a time interval

\footnotetext{
${ }^{1}$ Ruddiman, W. F., Kidd, R. B., and Thomas, E., et al., Init. Repts, DSDP, 94: Washington (U.S. Govt. Printing Office).

2 Addresses: (Ruddiman and Raymo) Lamont-Doherty Geological Observatory and Department of Geological Sciences of Columbia University, Palisades, New York 10964; (McIntyre) Lamont-Doherty Geological Observatory and Department of Geological Sciences of Columbia University, Palisades, New York 10964, and Queens College of the City University of lumbia University, Palisades, New York
New York, Flushing, New York 11367.
}

of about 3500 yr. The data are shown in Figure 2 and listed in Appendix A (Site 609) and B (Site 607). All measurements were made using the gasometric technique of Hulsemann (1966). Replicate analyses indicate a precision of $1-2 \%$. As indicated in Figure 2, the record at Site 609 was compiled by splicing analyses from Holes 609 and 609B. This was necessary to overcome large gaps caused by complications in the coring process (Ruddiman et al., this volume).

Both $\mathrm{CaCO}_{3}$ records are plotted versus time in Figure 3 . The time scales at Sites 609 and 607 are based on linear interpolation between available paleomagnetic datum levels (Clement and Robinson, this volume). These time scales are preliminary and will be adjusted in future work. Also shown plotted versus time in Figure 3 is the $\mathrm{CaCO}_{3}$ record from Hole 552A (Shackleton et al., 1984; Zimmerman et al., 1985).

All three records show the most marked change in $\mathrm{CaCO}_{3}$ near the boundary between the Gauss and Matuyama magnetic chrons at $2.47 \mathrm{Ma}$, with large-amplitude variations in carbonate percentage beginning at this level. This represents the first major development of glacial conditions in the subpolar gyre, including the initial delivery of ice-rafted detritus (Shackleton et al., 1984). However, the relative importance of this change in Ca$\mathrm{CO}_{3}$ percentage varies from site to site.

At Hole $552 \mathrm{~A}$, negligible $\mathrm{CaCO}_{3}$ variations occurred through most of the Gauss (as reported in Shackleton et al., 1984), followed by a pair of short $\mathrm{CaCO}_{3}$ minima at about $2.55 \mathrm{Ma}$, and then at $2.4 \mathrm{Ma}$ by a much more pronounced minimum that marks the beginning of largescale $\mathrm{CaCO}_{3}$ fluctuations.

At Site 607 , small variations ( $5 \%$ or less) were characteristic for a long interval prior to $3.15 \mathrm{Ma}$. A drift toward slightly lower $\mathrm{CaCO}_{3}$ values began around $3.15 \mathrm{Ma}$, 


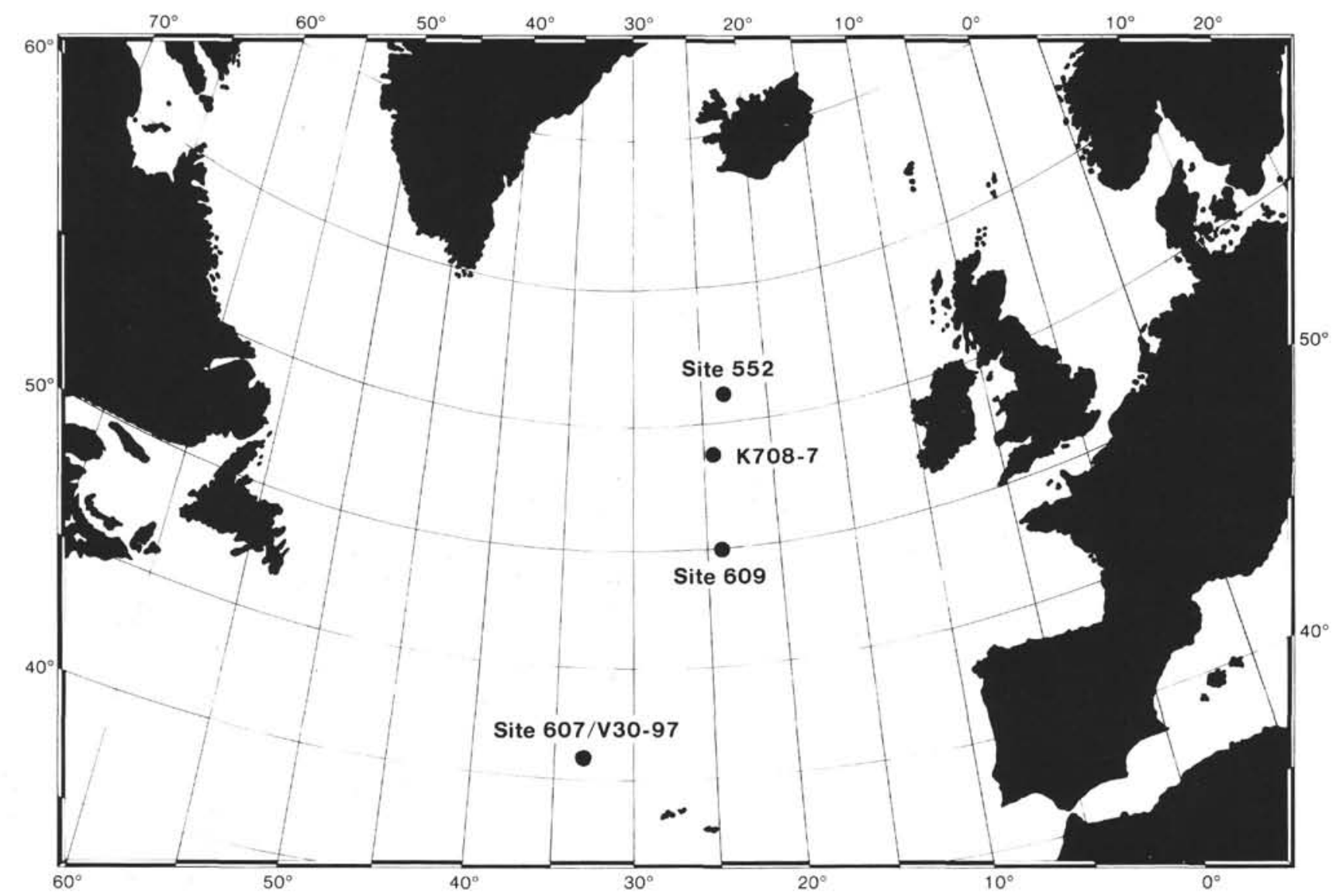

Figure 1. Locations of cores cited in text. Site 607 and Piston Core V30-97 are located at $41^{\circ} 00^{\prime} \mathrm{N}, 32^{\circ} 58^{\prime} \mathrm{W}$ ( $\left.3427 \mathrm{~m}\right)$. Site 609 is located at $49^{\circ} 53^{\prime} \mathrm{N}, 24^{\circ} 14^{\prime} \mathrm{W}(3883 \mathrm{~m})$. Site 552 is located at $56^{\circ} 03^{\prime} \mathrm{N}, 23^{\circ} 14^{\prime} \mathrm{W}$. Piston Core K708-7 is located at $53^{\circ} 56^{\prime} \mathrm{N}, 24^{\circ} 05^{\prime} \mathrm{W}$.

with a doubling in the amplitude of variation to about $10 \%$ at $2.7 \mathrm{Ma}$. The first major carbonate minimum occurred at $2.45 \mathrm{Ma}$, with a series of comparably strong $\mathrm{CaCO}_{3}$ oscillations $(25 \%)$ continuing until $1.95 \mathrm{Ma}$. The amplitude of $\mathrm{CaCO}_{3}$ variations then decreased somewhat, varying erratically between 15 and $25 \%$ from 1.9 to $1.35 \mathrm{Ma}$ before returning to intermediate $(25 \%)$ amplitudes from 1.35 to $0.8 \mathrm{Ma}$. Large-amplitude changes $(40-50 \%)$ began at $0.8 \mathrm{Ma}$ and continued through the rest of the Pleistocene.

At Site 609, a similar late Pliocene increase in the amplitude of $\mathrm{CaCO}_{3}$ variations occurred, with variations of less than $5 \%$ prior to $3.15 \mathrm{Ma}$, increasing to $15 \%$ to $20 \%$ by $2.6 \mathrm{Ma}$. This was, however, superimposed upon a drift toward lower mean $\mathrm{CaCO}_{3}$ values during this interval. More striking $\mathrm{CaCO}_{3}$ oscillations of $30 \%$ occurred at and after $2.55 \mathrm{Ma}$, followed by somewhat erratic but large-amplitude variations ( $50 \%$ or more) until about $0.85 \mathrm{Ma}$. The last $0.85 \mathrm{~m}$.y. have been characterized by large-amplitude $\mathrm{CaCO}_{3}$ variations (exceeding 70\%).

Several features of these records require comment. We will focus this discussion on three intervals during which important changes are evident in one or more of the Ca$\mathrm{CO}_{3}$ records: (1) the interval from 3.15 to $2.55 \mathrm{Ma}$, during which two of the three records show declining Ca$\mathrm{CO}_{3}$ values; (2) the abrupt beginning of strong, periodic $\mathrm{CaCO}_{3}$ variations, and particularly the deeper $\mathrm{CaCO}_{3}$ minima, around 2.55 to $2.47 \mathrm{Ma}$; and (3) the increase in amplitude of $\mathrm{CaCO}_{3}$ oscillations in the last $0.85 \mathrm{~m}$.y.

$\mathrm{CaCO}_{3}$ changes in this area during the late Pleistocene were mainly caused by variable suppression of biogenic productivity due to salinity stratification and sea ice (Ruddiman and McIntyre, 1976), and by variable deposition of ice-rafted detritus (Ruddiman, 1977). In order to explain the very large late Quaternary changes in $\mathrm{CaCO}_{3}$ content without comparably large changes in sedimentation rate in most North Atlantic cores, the effects of these two factors on the flux of sediment to the seafloor must generally be opposed and nearly balanced through time.

Dissolution of $\mathrm{CaCO}_{3}$ is generally regarded as a second-order factor in the high-amplitude $\mathrm{CaCO}_{3}$ changes of the late Pleistocene North Atlantic. However, for the smaller-amplitude $\mathrm{CaCO}_{3}$ variations in the earlier parts of the Pleistocene and late Pliocene, dissolution may play a first-order role and must be considered.

The three sites have different $\mathrm{CaCO}_{3}$ responses across the interval from 3.15 to $2.55 \mathrm{Ma}$. The strongest response is the marked drift to lower values at deep Site 609 $(3883 \mathrm{~m})$. The drift to lower values is more subtle at the intermediate Site $607(3427 \mathrm{~m})$ and there is no drift at the shallow Site $552(2311 \mathrm{~m})$. This progression suggests that the trend toward lower $\mathrm{CaCO}_{3}$ from 3.15 to $2.55 \mathrm{Ma}$ is largely a result of depth-dependent dissolution. 


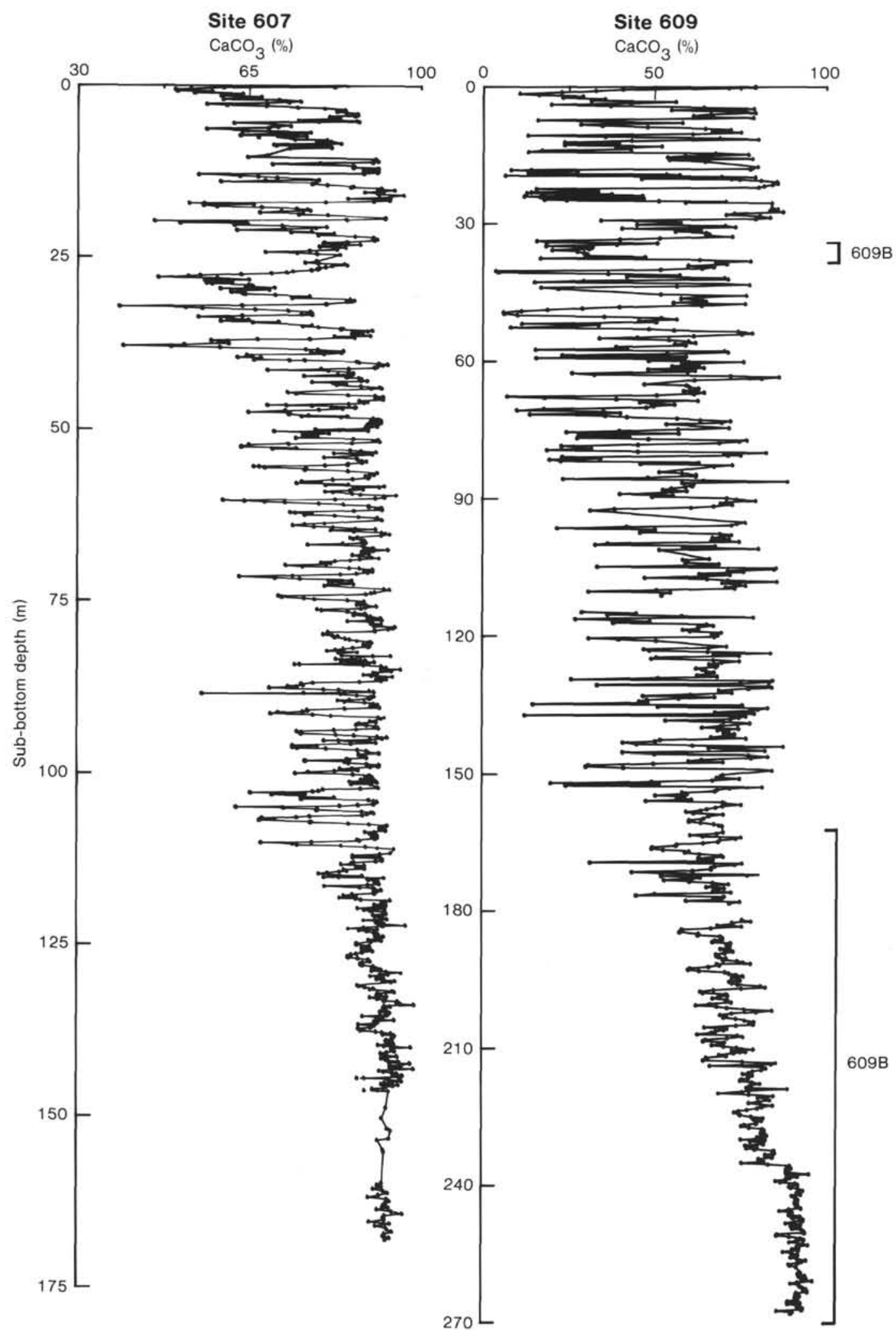

Figure 2. Variations in $\mathrm{CaCO}_{3}$ percentage at Sites 607 and 609 plotted versus sub-bottom depth. Note the expanded amplitude scale for the $\mathrm{CaCO}_{3}$ values at Site 607 . 


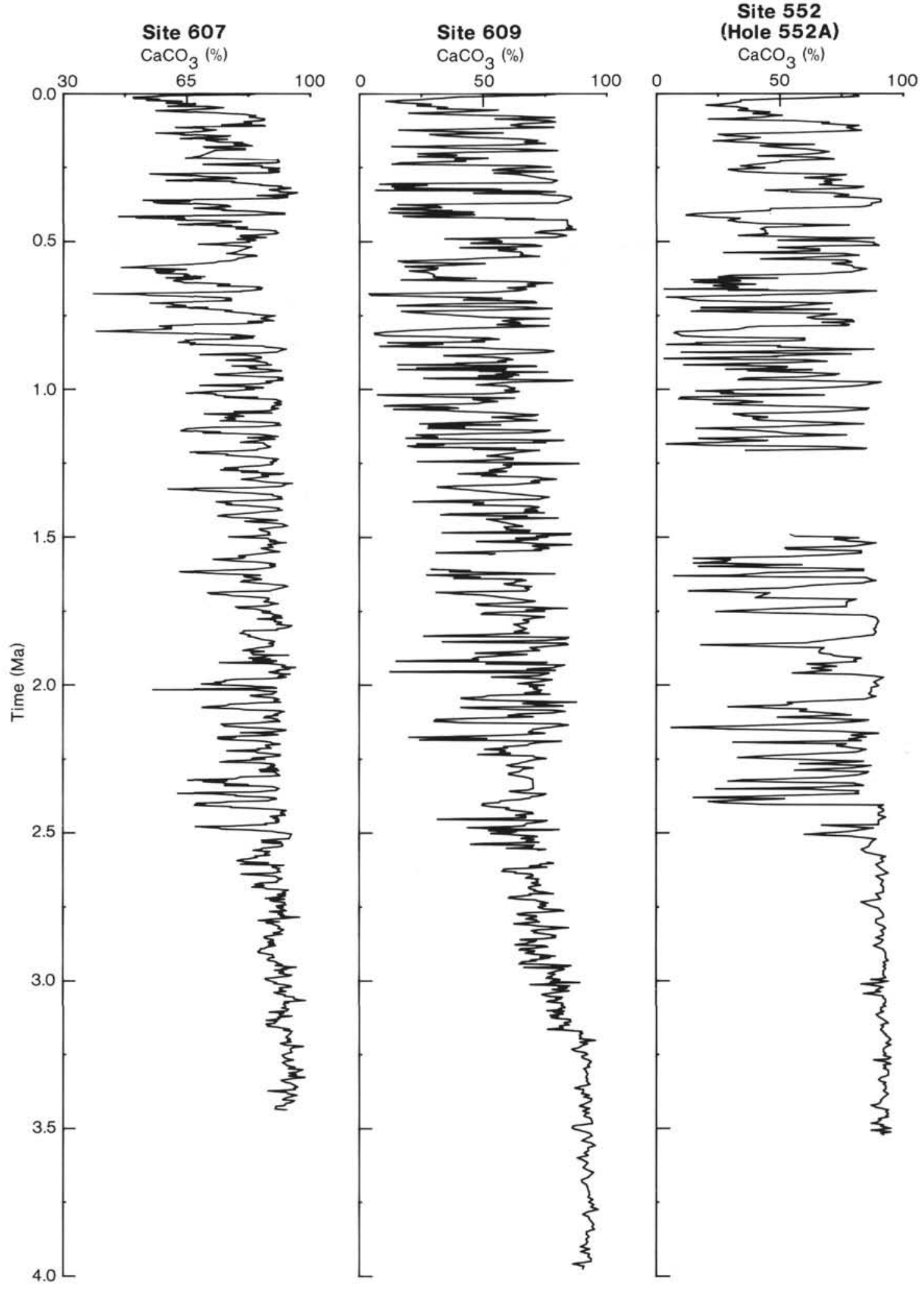

Figure 3. Variations in $\mathrm{CaCO}_{3}$ percentage at Sites 607, 609, and 552 plotted against time. Hole 552A data are from Shackleton et al. (1984) and Zimmerman et al. (1985). 
We examined the sand fraction (coarser than $0.062 \mathrm{~mm}$ ) of those samples at Sites 609 and 607 with the lowest $\mathrm{CaCO}_{3}$ values in the interval from 3.15 to $2.55 \mathrm{Ma}$. We found no ice-rafted grains in any sample below the 2.55-Ma level. The only noncarbonate detritus present in the coarse fraction was pyrite. This eliminates dilution by ice rafting as a causal factor of the gradual Ca$\mathrm{CO}_{3}$ drift. At this point it is not possible to eliminate productivity suppression as an explanation, but the depthdependent trends argue that dissolution is the primary factor. It is also possible that an increased local influx to Site 609 of fine detritus derived from nearby Maury Channel (Ruddiman, 1972) reduced the carbonate percentages at this site during the Gauss chron.

Thus our results confirm the observation first made by Shackleton et al. (1984) that the initiation of major Northern Hemisphere glaciation occurred near the Gauss/ Matuyama boundary. The first substantial reductions in $\mathrm{CaCO}_{3}$ and minor influxes of ice-rafted sand occurred just below the paleomagnetic boundary between 2.57 and $2.55 \mathrm{Ma}$ in all three records, but it is only at or just above the Gauss/Matuyama boundary at $2.47 \mathrm{Ma}$ that abundant ice-rafted sand appears. All three sites record deep $\mathrm{CaCO}_{3}$ minima with abundant ice-rafted sand grains just after $2.47 \mathrm{Ma}$. This change affected the entire eastcentral North Atlantic from $56^{\circ} \mathrm{N}$ southward to $41^{\circ} \mathrm{N}$, the effective southern limit of abundant ice rafting during the Pleistocene (Ruddiman, 1977).

The three sites also show interesting differences in $\mathrm{CaCO}_{3}$ trends during the later Pleistocene (late Matuya$\mathrm{ma}$ and early Brunhes chrons). There is an increased amplitude of variation during the last $0.8 \mathrm{~m}$.y. at Site 607 (especially in the late Matuyama and early Brunhes), a similar but somewhat less obvious increase in $\mathrm{CaCO}_{3}$ variations during the last $0.85 \mathrm{~m}$.y. at Site 609 , and, in contrast, a decrease in range of $\mathrm{CaCO}_{3}$ changes at Site 552 during the last $0.6 \mathrm{~m}$.y. This pattern suggests that the very large-scale ice-volume oscillations that occurred in the Brunhes epoch (Shackleton and Opdyke, 1973) tended to send ice-rafted debris farther south than those in the Matuyama, thus shifting the largest relative impact to the south (Site 607) and depressing the relative impact farther north (Site 552). Kent et al. (1971) also found higher percentages of ice-rafted sand in the North Pacific during the last million years than in the earlier Matuyama. This trend during the last million years could also reflect a relatively increased role of the Laurentide ice sheet, which would probably have the largest impact at westernmost Site 607 and the smallest effect at easternmost Site 552.

\section{OXYGEN ISOTOPIC VARIATIONS AT SITE 607 (1.80-0 Ma)}

Benthic foraminifers were picked for oxygen isotope analysis from the same suite of samples run for $\mathrm{CaCO}_{3}$ percentage at 4500 -yr. intervals (on the average) over the time span from $1.80 \mathrm{Ma}$ to the present. The two species used were Uvigerina peregrina and Cibicides (also known as Planulina) wuellerstorfi. Uvigerina is assumed to be in oxygen isotopic equilibrium with ambient sea water; Cibicides is adjusted by $+0.64 \%$ to bring it into pre- sumed equilibrium (Shackleton, 1977). The unadjusted values are listed in Appendix C. Figures 4, 5, and 6 show adjusted values. For the upper part of the records (above the oxygen isotopic stage 8/7 boundary in Figs. 5 and 6), we plotted previously published oxygen isotopic values measured in Piston Core V30-97 at the same location as Site 607 (Ruddiman and McIntyre, 1981).

The isotopic data from Site 607 are plotted versus subbottom depth in Figure 4. All major oxygen isotopic stages from stages 8 to 22 are clearly visible. In general, their relative amplitudes are comparable to those in the stacked isotopic record published in Imbrie et al. (1984). For example, glacial stages 12 and 16 have the heaviest $\delta^{18} \mathrm{O}$ values. Stages 11 and 12 appear to occur twice in the section because of double coring (Ruddiman, Cameron, and Clement, this volume).

The absolute amplitude of the isotopic signal from Site 607 is very large $(\sim 2.5 \%)$. This may result in part from splicing together data from two core sites. However, the fact that the amplitudes are still unusually large within each of the two parts of the record indicates that changes in bottom-water temperature have significantly overprinted the ice-volume fluctuations at this site.

A preliminary time scale for the spliced record of Core V30-97 and Site 607 was derived from oxygen isotopic and paleomagnetic data. For the upper 0.25 m.y. (spanned by Core V30-97), we used the time scale published by Ruddiman and McIntyre (1984). For the interval below $0.25 \mathrm{Ma}$ in the record from Site 607 , we interpolated ages between the isotopically defined 0.25 -Ma level and the paleomagnetic boundaries from Clement and Robinson (this volume). Oxygen isotopic data plotted to this time scale and spanning the last $1.0 \mathrm{Ma}$ are shown in Figures 5 and 6.

\section{PLANKTONIC FORAMINIFERAL VARIATIONS AT SITE 607 (1.90-0 Ma)}

Figure 4 shows percentage abundance fluctuations of four key species of planktonic foraminifers over the upper $85 \mathrm{~m}$ of record at Site 607 . The counts are based on aliquots averaging slightly over 300 individuals larger than $0.149 \mathrm{~mm}$ from samples taken every $15 \mathrm{~cm}$, equivalent to a time interval of about $3500 \mathrm{yr}$. All counts are listed in Appendix C. The same data are plotted in Figure 5 to the time scale described in the last section. The Site 607 counts extend from 0.25 to $1.90 \mathrm{Ma}$ (Fig. 5). For the portion of the record above $0.25 \mathrm{Ma}$ in Figure 5, we show counts from Core V30-97 (Ruddiman and McIntyre, 1981).

Particularly noteworthy among the trends shown in Figure 5 are: (1) the pulses of increased abundance of the cold, polar-water indicator Neogloboquadrina pachyderma (sinistral, or left-coiling) between 1.05 and $0.8 \mathrm{Ma}$, and at $0.68 \mathrm{Ma}, 0.45 \mathrm{Ma}$, and $0.35 \mathrm{Ma}$, with intervals of near-zero abundance between 1.9 and $1.7 \mathrm{Ma}$ and between 1.25 and $1.1 \mathrm{Ma}$ and generally lower percentages between 0.65 and $0.47 \mathrm{Ma}$ and between 0.34 and $0.07 \mathrm{Ma}$; (2) a progressive increase in the abundance of the cool subpolar species Globigerina bulloides throughout the length of the record; (3) large variations of the temperate North Atlantic Drift indicator, Globorotalia inflata, 

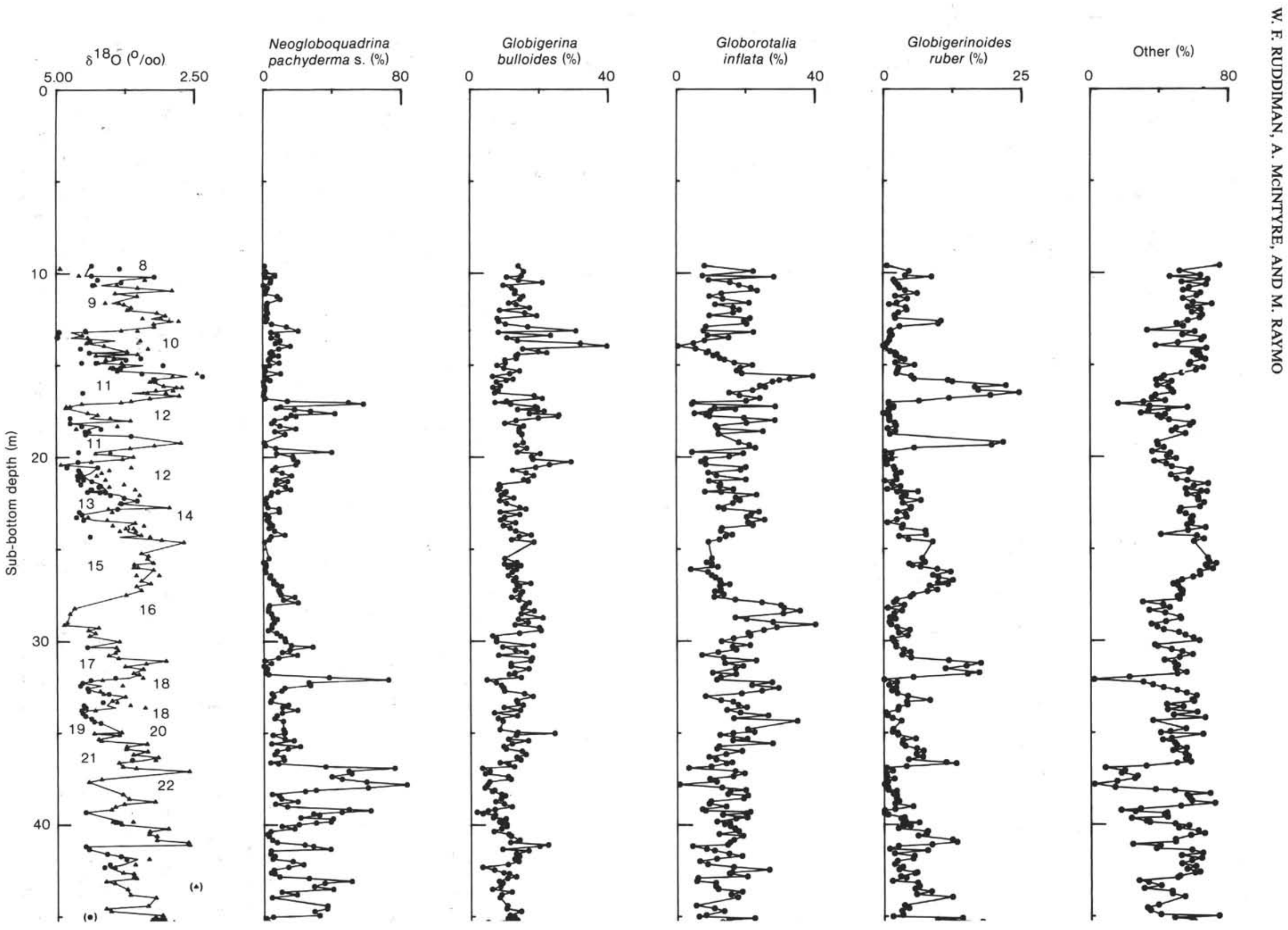

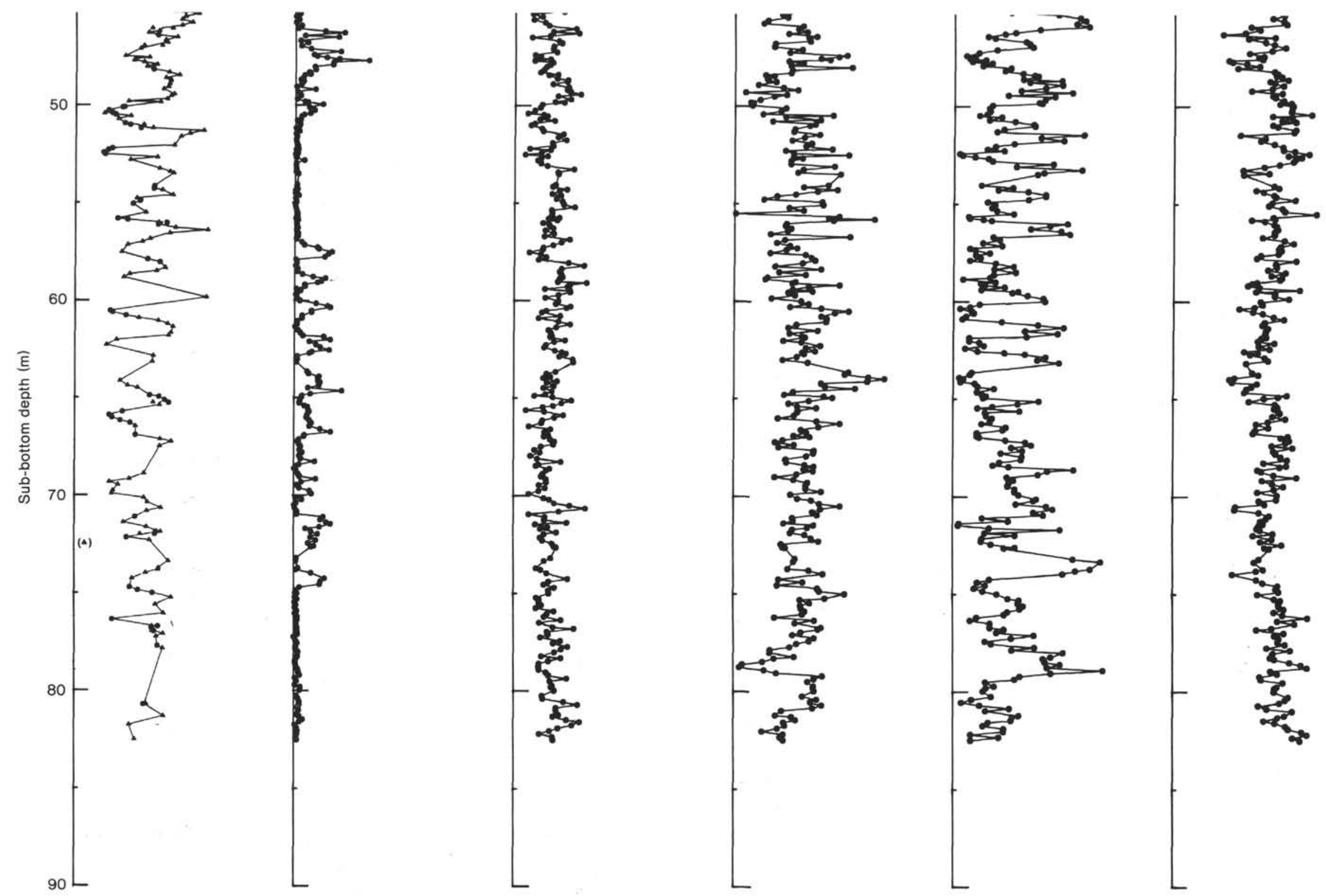

igure 4. Percentage abundance of four species of planktonic foraminifers and percentage abundance of all other species combined, plotted versus sub-bottom depth at Site 607 over the last 1.9 m.y. Also shown are oxygen isotopic data from Site 607 based on values derived from benthic foraminifers Uvigerina $(\bullet)$ and Cibicides $(\boldsymbol{\Delta})$; values in parentheses were not included in the continuous record (the solid line). Numbers are $\delta^{18} \mathrm{O}$ stage designations. 
V30-97/Site 607
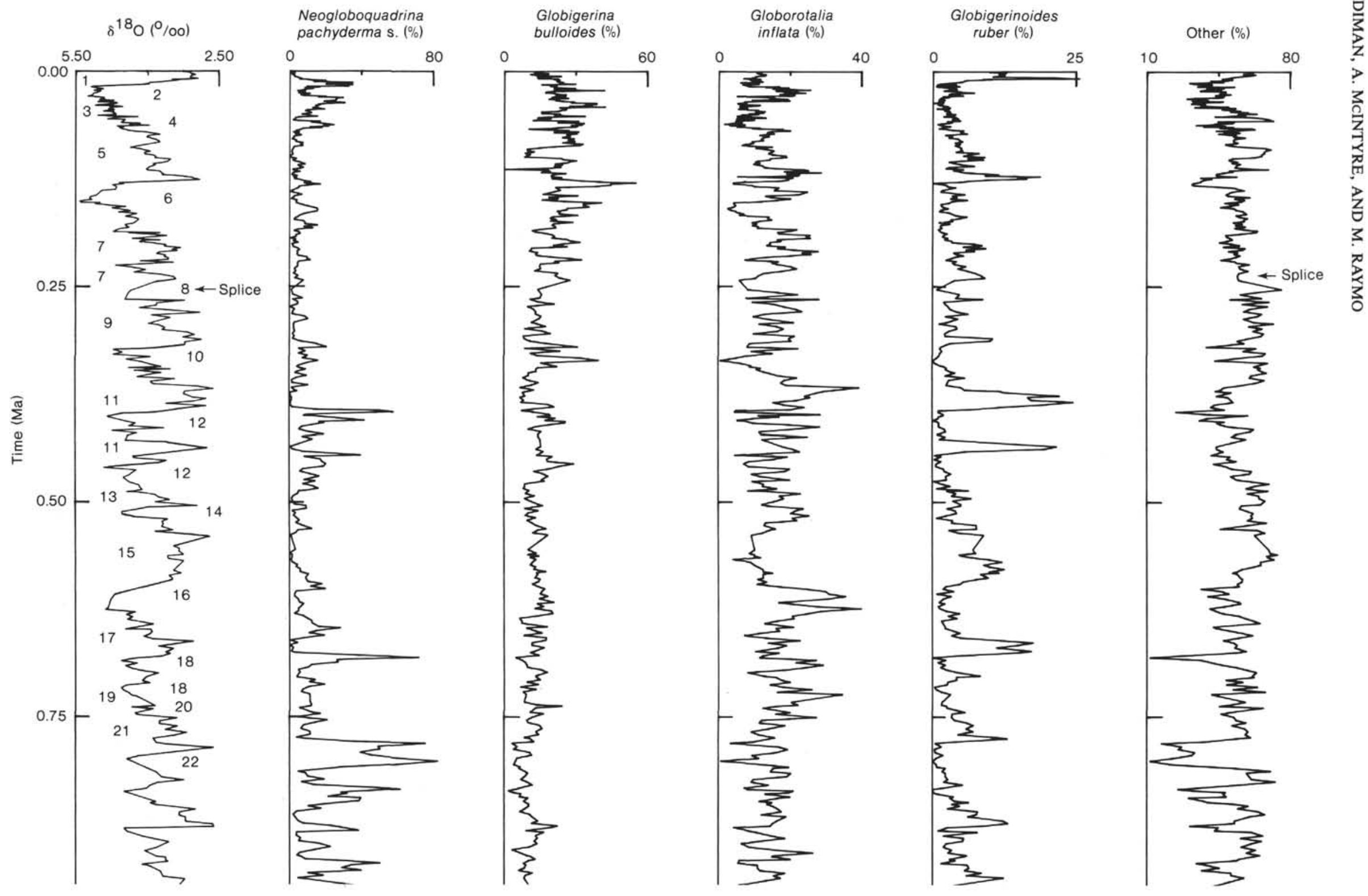


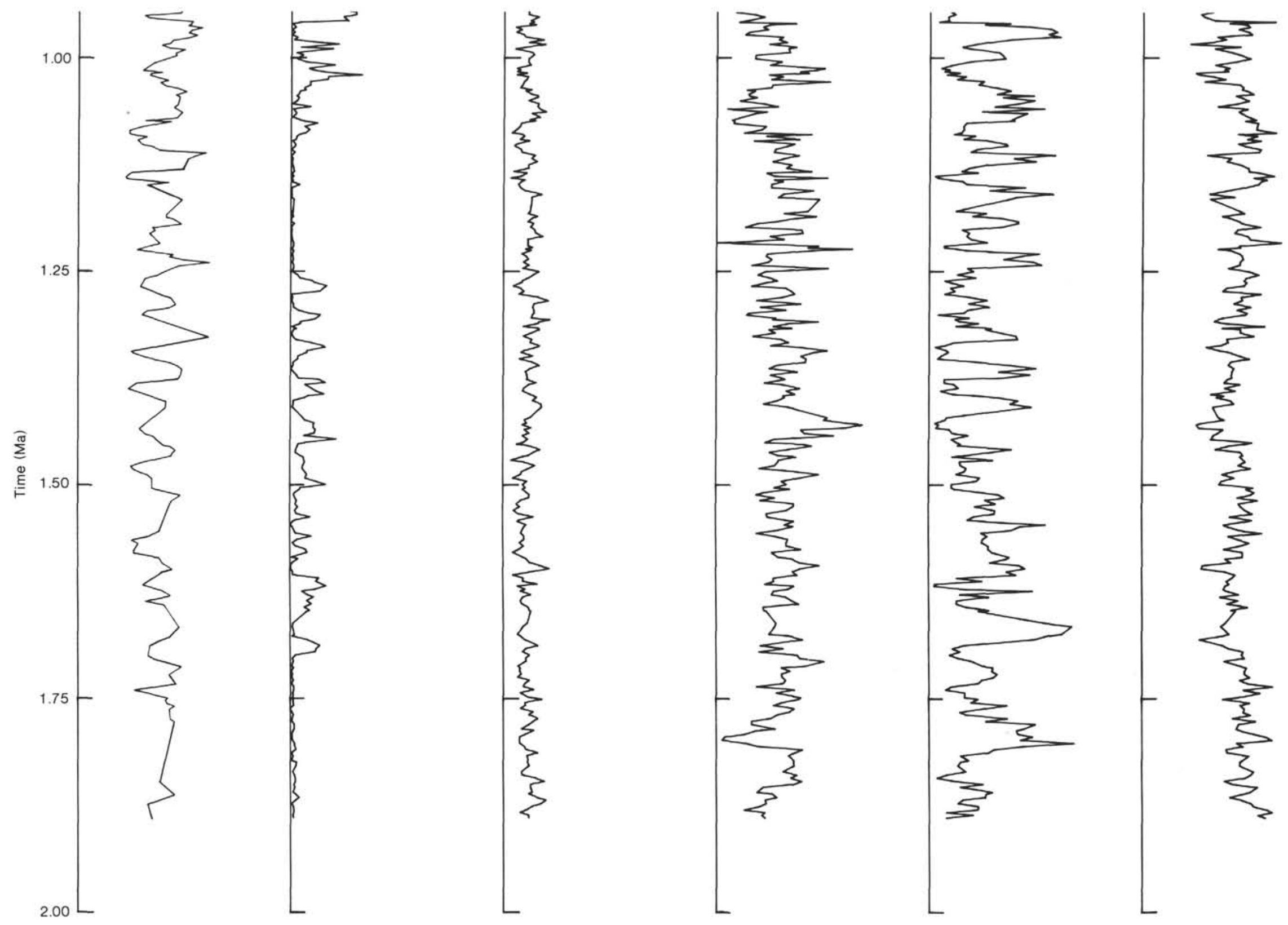

Figure 5. Percentage species abundance and oxygen isotopic data from Site 607 and Core V30-97 spliced at $0.25 \mathrm{Ma}$ and plotted against time. 
with generally somewhat reduced values after $1.1 \mathrm{Ma}$, and a suggestion of lower values prior to $1.8 \mathrm{Ma}$; and (4) a reduction in the mean abundance and range of variation of the warm subtropical species Globigerinoides ruber (white) after $0.95 \mathrm{Ma}$, except for a few very large maxima in the Brunhes chron.

No-analog abundances do not occur in the species percentage data from the samples in this record (Figs. 4, 5). Ruddiman, Shackleton, et al. (in press) have found no-analog faunas in the $>1.2$ to $1.1 \mathrm{Ma}$ interval from Hole 552A about $1700 \mathrm{~km}$ to the north-northeast of Site 607 (Fig. 1). During portions of this interval, G. inflata percentages at Hole 552A exceeded by 5 to $10 \%$ the $40 \%$ maximum range found in North Atlantic core tops (Kipp, 1976). Throughout the same interval, $N$. pachyderma (s.) values dropped to nearly zero at Hole $552 \mathrm{~A}$. Because of a record gap between 1.4 and $1.2 \mathrm{Ma}$ at Hole 552A, it was not possible to define the beginning of this anomalous interval at that site, other than to constrain it as older than $1.2 \mathrm{Ma}$ and younger than 1.4 Ma. In the record at Site $607, G$. inflata values stayed within the $40 \%$ limit from 1.2 to $1.1 \mathrm{Ma}$, so that a noanalog condition was not attained. $N$. pachyderma (s.) percentages from Site 607 indicate that the interval of near-zero abundance lasted from 1.25 to $1.1 \mathrm{Ma}$. A similar interval occurred prior to $1.7 \mathrm{Ma}$ (see also Raymo, Ruddiman, and Clement this volume).

\section{SEA-SURFACE TEMPERATURE VARIATIONS AT SITE 607 (1.90-0 Ma)}

Estimated winter sea-surface temperature over the last 1.90 m.y. at Site 607 based on the counts shown in Figures 4 and 5 are shown plotted versus time in Figure 6. These estimates (listed in Appendix C) are based on recently developed transfer function F13 $\times 5$ (Ruddiman and Esmay, this volume). This transfer function was devised specifically to deal with the large number of samples required to analyze $\mathrm{HPC}$ records in detail. It requires the recognition of just the four species shown in Figures 4 and 5, and all remaining species are lumped into a fifth category-“Other".

Although the statistical measures of equation performance are inevitably degraded somewhat from those of the widely used North Atlantic equation F13 of Kipp (1976), the standard errors of estimate $\left(2.2^{\circ} \mathrm{C}\right.$ in summer and $1.9^{\circ} \mathrm{C}$ in winter) are still relatively small for an area where the total range of Quaternary SST variation is in excess of $15^{\circ} \mathrm{C}$. The new equation captures the major trends and basic rhythms of cyclical SST change in the late Pleistocene as well as the full F13 equation in this area (Ruddiman and Esmay, this volume).

For the upper $0.25 \mathrm{~m} . \mathrm{y}$. of record shown in Figure 6, the sea surface temperature in winter (SSTw) estimates are based on the counts in Core V30-97 published by Ruddiman and McIntyre $(1981,1984)$. In order to make these data consistent with the Site 607 counts, they were reprocessed through transfer function $\mathrm{F} 13 \times 5$, which recognizes only the reduced number of species mentioned. Only the winter-season (SSTw) estimates are shown, because this was the best-simulated season and because the trends in both seasons are quite similar.
The SSTw curves at Site 607 (Fig. 6) are complex, with significant variations apparent at both very long and relatively short wavelengths. A prolonged warm interval occurred prior to $1.05 \mathrm{Ma}$, but with significant variations at both long and short wavelengths. A trend is apparent at the longer $(500,000-600,000 \mathrm{yr}$.) wavelength from intermediate temperatures at the bottom of the record (1.90 Ma) to an SST maximum at around 1.651.60 Ma, an SST minimum at about 1.40-1.30 Ma, and an SST maximum centered at about 1.15-1.10 Ma, prior to a prominent step-function cooling at around $1.0 \mathrm{Ma}$.

The part of the long-wavelength structure showing warmth before $1.7 \mathrm{Ma}$ and between 1.25 and $1.1 \mathrm{Ma}$ may be due to bias in the transfer function: these are the intervals in which Neogloboquadrina pachyderma (s.) is absent. Farther north, this absence creates severe no-analog conditions at Site 552 (Ruddiman et al., in press). Because N. pachyderma (s.) is less abundant at Site 607 even in most peak-glacial intervals, its absence appears only to impart a small warm bias to the SST estimates without totally invalidating the use of the transfer function.

Shorter-period variations around 40,000 yr. during the Matuyama are superimposed on these longer-term SSTw changes and are also evident in the $\mathrm{CaCO}_{3}$ record. Recent analysis of both SSTw and $\mathrm{CaCO}_{3}$ percentage for the Site 607 record between 2.47 and $0.735 \mathrm{Ma}$ shows a very powerful concentration of variance at the orbital obliquity period of 41,000 yr. (Ruddiman, Raymo, et al., in press).

The abrupt shift to colder conditions after 1.0 Ma and the positioning of the coldest estimates in the $0.8-$ $0.7 \mathrm{Ma}$ section of Figure 6 do not reflect bias in the transfer function. The $\mathrm{CaCO}_{3}$ record from Site 607 independently shows nearly identical trends, indicating that this is a valid local climatic signal. Pulses of increasingly low SSTw values occurred from 1.05 to $0.8 \mathrm{Ma}$, and again at $0.68 \mathrm{Ma}$, with relative warmth from 0.67 to $0.5 \mathrm{Ma}$, and periodic coolings after $0.5 \mathrm{Ma}$.

The strongest cold pulses in the record younger than $1.05 \mathrm{Ma}$ are coincident with maxima in the polar species N. pachyderma (s.) (Figs. 4, 5). Some of the more moderate cold pulses are correlated with maxima in Globigerina bulloides, although the link between this species and the SST estimates is otherwise not very strong.

The general prevalence of warmth prior to $0.95 \mathrm{Ma}$, as well as the scattered pulses of extreme warmth in the generally colder interval after that date, are both linked to high abundances of the subtropical species $G$. ruber (Figs. 4, 5). Values of that species in excess of $15 \%$ and ranging up to $25 \%$ produce SST estimates in excess of $24^{\circ} \mathrm{C}$ and ranging up to $26.5^{\circ} \mathrm{C}$.

One interesting trend in the SST curve is the tendency for the lowest SST estimates late in several of the glacial isotopic stages to be immediately followed by the highest SST estimates early in the succeeding interglacial isotopic stages (Fig. 6). This occurs most notably at the following isotopic stage boundaries: $10 / 9(0.337 \mathrm{Ma}) ; 12 /$ 11 (0.421 Ma); 18/17 (0.687 Ma); and 22/21 (0.788 Ma), but it is also evident at the $6 / 5$ boundary $(0.127 \mathrm{Ma})$. Although a small part of this pattern is related to inade- 
quacies in the transfer function used to make the SSTw estimates (Ruddiman and Esmay, this volume), the striking percentage changes in the cold-indicator species $N$. pachyderma (s.) and warm indicator $G$. ruber (Figs. 5, 6) confirm that extremes of warmth immediately followed extremes of cold.

Finally, the SSTw curve at Site 607 differs rather strikingly from that at Hole 552A, also shown in Figure 6. Except for the previously mentioned no-analog conditions from 1.2 to $1.1 \mathrm{Ma}$ at Hole $552 \mathrm{~A}$, the curve at Hole $552 \mathrm{~A}$ tends to oscillate repetitiously between similar extremes of warmth and cold. The range of SST oscillation at Hole 552A increased progressively from the smaller variations typical a million years ago to the larger variations of the last $0.45 \mathrm{~m}$.y., but this increase in amplitude was slow and gradual.

In contrast, the SST record at Site 607 is abruptly punctuated by unusually cold and warm pulses; it shows oscillations at very long wavelengths; and it is subject to larger base-line shifts from one range of variation to another. All of these effects create a more complex pattern than the more rhythmic trends evident at Hole 552A.

Several features in the $\mathrm{CaCO}_{3}$ curve at Site 607 are similar to those in the SSTw curve (Fig. 6). The unusually deep $\mathrm{CaCO}_{3}$ minima in oxygen isotopic stages 22 , 18,12 , and 10 match the unusually cold SST estimates at those levels, although the $\mathrm{CaCO}_{3}$ minimum in stage 10 lacks the full intensity of the SSTw minimum. These strong carbonate minima thus validate the unusually cold SSTw values.

\section{CORING PROBLEMS}

Although the data reported here for Sites 607 and 609 are valid when plotted against sub-bottom depth in each hole at each site, the time series plots are not final. Several problem areas must be examined more closely before the records of $\mathrm{CaCO}_{3}, \delta^{18} \mathrm{O}$, foraminiferal species percentages, and estimated SST can be accepted as accurate time series and analyzed with the standard signalprocessing techniques (spectral analysis, filtering, coherency and cross-correlation analysis).

The major problem is the existence of gaps in the record at breaks between cores. Correlation lines shown in Ruddiman et al. (this volume) suggest that $75 \mathrm{~cm}$ or more of material may be missing across some core breaks at these sites. At the mean Pleistocene sedimentation rates of 45 and $75 \mathrm{~m} . / \mathrm{m} . y$. at Sites 607 and 609 , this would equate to maximum time gaps at the core breaks of about 17,000 yr. at Site 607 and 10,000 yr. at Site 609. These gaps are being rectified by sampling comparable sections in the alternate holes at these sites.

\section{DISCUSSION}

Site 607 is located beneath surface waters with the largest temperature changes (glacial to interglacial ranges of 12 to $15^{\circ} \mathrm{C}$ ) in the world during the late Pleistocene (CLIMAP, 1981; Ruddiman and McIntyre, 1984). The 1.90-m.y. SSTw record of the entire Pleistocene shown in Figure 6 indicates even larger changes of up to $20^{\circ} \mathrm{C}$. The relative warmth indicated for most of the Matuyama chron is not surprising; oxygen isotopic evidence sug- gests smaller ice volume (both mean and glacial-maximum values) at that time (Shackleton and Opdyke, 1973).

Although the faunal and SST time series in Figures 5 and 6 are not final, the pulses of very cold SSTw at roughly $0.8 \mathrm{Ma}$ (isotopic stage 22) and $0.68 \mathrm{Ma}$ (stage 18) merit additional comment, because they far exceed the lowest glacial SST values observed in the two large-scale glacial cycles of the last $250,000 \mathrm{yr}$. The cold pulses at $0.45 \mathrm{Ma}$ (stage 12) and $0.35 \mathrm{Ma}$ (stage 10) are also slightly larger than the late Pleistocene glacial values.

We do not believe that these SST estimates are erroneous. The raw species data in Figure 5 are consistent with the estimated SSTw values, with very high $N$. pachyderma (s.) percentages (as much as $90 \%$ ) requiring very low temperatures. In addition, the cold SST pulses are corroborated by correlative lows in the $\mathrm{CaCO}_{3}$ percentage (Fig. 6). Similarly, the high $G$. ruber percentages (as much as $25 \%$ ) following some of the succeeding deglaciations (Fig. 5) require high interglacial SST values.

Following the line of reasoning used by Ruddiman and McIntyre $(1981,1984)$ to explain the climatic response of North Atlantic surface waters over the last 250,000 yr., the origin of these cold-warm pulses probably lies in one of two factors: (1) changes in forcing of the highlatitude ocean by nearby ice sheets or (2) changes in northward advection of warm tropical and subtropical surface waters due to climatic interactions that are physically remote from the ice sheets.

From evidence in Ruddiman and McIntyre (1984), icesheets can force the North Atlantic response in two ways: (1) at latitudes north of $50^{\circ} \mathrm{N}$, via the immediate effects of the ice on the atmosphere, and of the cold lower-atmospheric winds on the surface ocean (Manabe and Broccoli, 1985), with little or no lag of the SST response, behind ice volume; and (2) at slightly lower latitudes (35$50^{\circ} \mathrm{N}$ ), via the iceberg-meltwater flow, with a lag of several thousand years of the surface-ocean temperatures behind ice volume. To explain the anomalously low SST pulses observed in Figures 5 and 6, the first explanation would require unusually large ice sheets; the second would require unusually rapid deglaciations.

We see no strong evidence in support of the first icesheet explanation. There is no obvious correlation between the lowest SST values at Site 607 and the heaviest $\delta^{18} \mathrm{O}$ values in the stacked isotopic record used as an icevolume proxy by Imbrie et al. (1984). The lowest SST estimates in the Site 607 record fall in stages 22 and 18, with less prominent low values in stages 10,12 , and 6 . In the oxygen isotopic record of Imbrie et al. (1984), the heaviest $\delta^{18} \mathrm{O}$ values occur in stages $16,2,6$, and 12 . Thus the lowest SST values do not fall at the times of largest ice volume; stages 6 and 12 are the only intervals common to both sets.

A second possibility is that during isotopic stages 22 , 18,12 , and 10 there was an unusually large Laurentide ice sheet, which presumably is more important than other ice sheets in controlling the local temperature/salinity response of this part of the North Atlantic. But this would require that all other ice sheets be much smaller to compensate for the enhanced Laurentide effect in the global oxygen isotopic record at these times, which seems unlikely. 

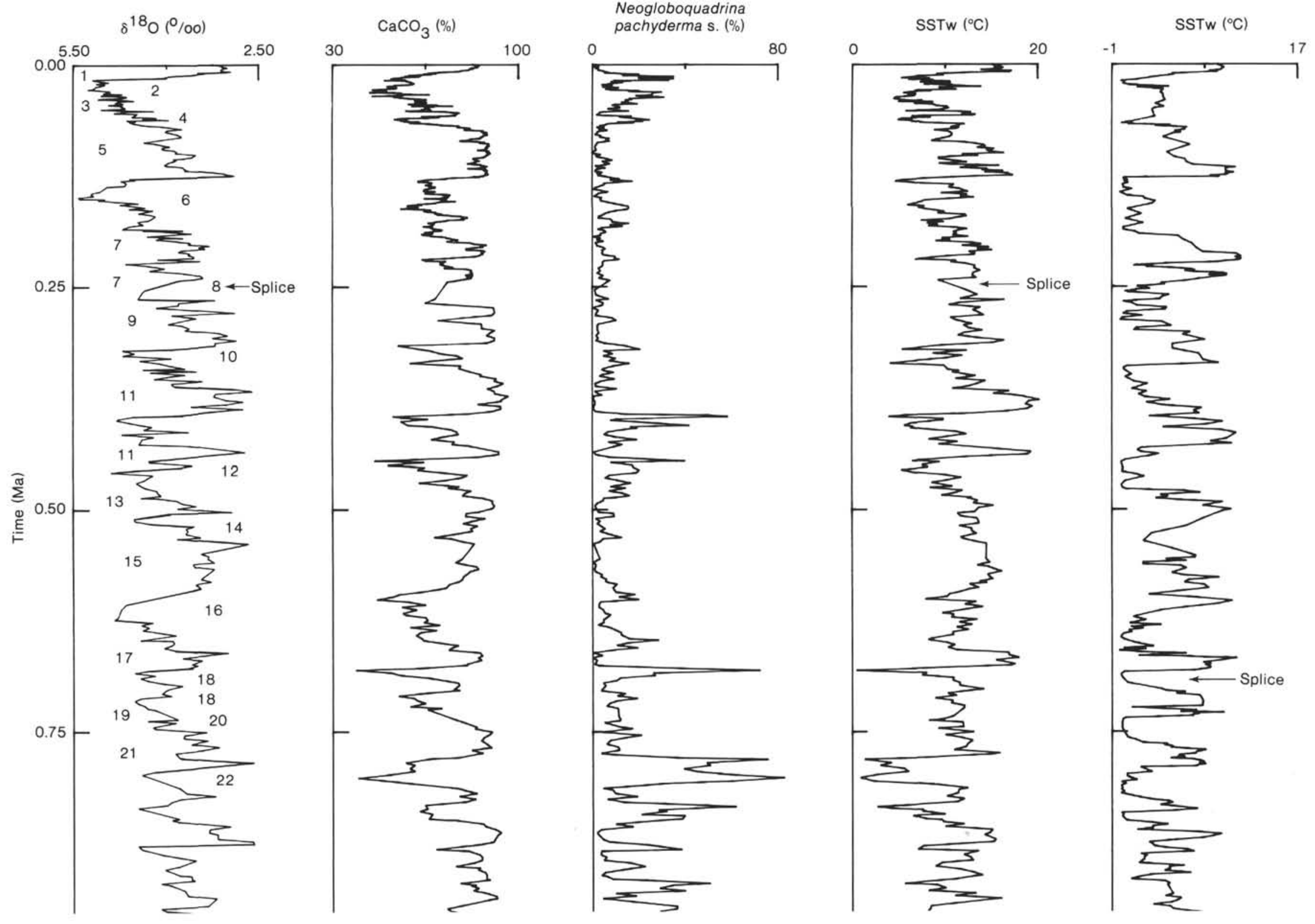

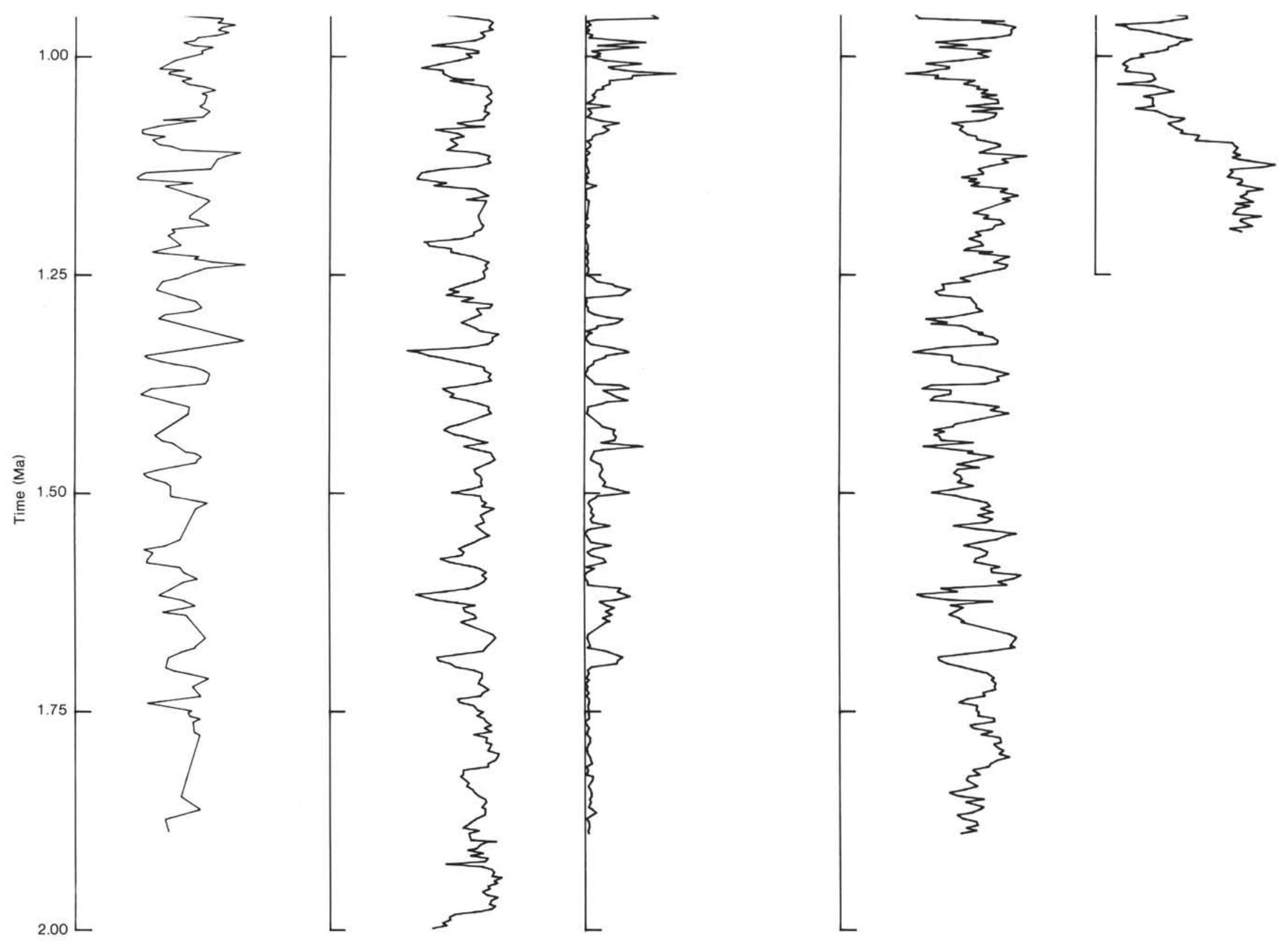

Figure 6. Oxygen isotopic, $\mathrm{CaCO}_{3}$, and $N$. pachyderma (s.) percent data from the spliced Site 607/Core V30-97 record plotted versus time, along with winter sea-surface temperature (SSTw) in this record and in the spliced Hole 552A/Core K708-7 record. 
Evidence in support of the second of the two ice-related explanations - faster rates of deglaciation, causing a more concentrated influx of icebergs and meltwater into the North Atlantic-is also weak. Faster deglaciation could occur as a response to a larger rate of insolation forcing of the ice sheets. However, data in Berger (1977) indicate that none of the insolation parameters thought to be causal in forcing deglaciations (eccentricity maxima, obliquity minima, or precessional terms) show unusual values uniquely correlative with the cold pulses noted above. Nor are actual values of incoming insolation at the latitudes of the ice sheets $\left(45-65^{\circ} \mathrm{N}\right)$ unusual in any way at or near the times of these SST anomalies. Thus no anomalous insolation forcing of ice toward deglaciation exists at these times.

A second possibility linked to the rate of deglaciation is that there was a maximum response at these times of one or more of the feedback factors within the climate system that contribute to rapid deglaciations. The most likely feedback factors are: bedrock rebound, marine calving, increased $\mathrm{CO}_{2}$, and moisture starvation. It is, however, difficult to imagine why these factors would operate more vigorously during the particular deglaciations in question, particularly at isotopic stages $22 / 21$ and 18 / 17. Moreover, the rate of operation of all of these feedbacks is ultimately tied directly to ice volume, which, as already noted, was not unusually large in global terms at these times.

It is impossible to evaluate at this time the other likely explanation (changes in northward advection of warm, low-latitude waters) because of the scarcity of long-term SST records in critical low-latitude locations. The only available time series covering the last million years in the low-latitude Atlantic are coarse-resolution SST estimates (average sampling interval 17,000 yr.) from Briskin and Berggren (1975) and pretransfer function data from Ruddiman (1971). Neither of these studies pinpoints anything unusual about the intervals in question.

Still, the contrast between the SST signals at Sites 552 and 607 (Fig. 6) is instructive. The SST record at Hole $552 \mathrm{~A}$ can be attributed to relatively direct control by the Northern Hemisphere ice-volume signal evident in oxygen isotopic curves, with larger ice volume forcing lower temperatures (Ruddiman et al., in press). In contrast, the preliminary SST record from Site 607 is not totally explainable in terms of Northern Hemisphere ice volume. And it is difficult to imagine Laurentide ice decoupling entirely from the other high-latitude ice sheets. Therefore, the explanation for the trends at Site 607 may lie in forcing from low latitudes. Future ODP drilling on Leg 108 should help address this problem.

\section{ACKNOWLEDGMENTS}

We acknowledge the assistance of Joanne Munkelt and Denise Oswald in laboratory analyses, Ann Esmay in data processing, and Beatrice Rasmussen in manuscript preparation. We thank Joe Morley, William Showers, and Bill Curry for critical reviews. This research was supported by NSF grant OCE82-19862 from the Submarine Geology and Geophysics Program of the Ocean Science Section of the National Science Foundation. We acknowledge the DSDP core curator for assistance in obtaining samples. This is Lamont-Doherty Geological Observatory Contribution No. 3989.

\section{REFERENCES}

Berger, A. L., 1977. Long-term variations of caloric insolation resulting from the earth's orbital elements. Quat. Res., 9:139-167.

Briskin, M., and Berggren, W. A., 1975. Pleistocene stratigraphy and quantitative paleo-oceanography of tropical North Atlantic core V16-205. In Saito, T., and Burckle, L. (Eds.), Late Neogene Epoch Boundaries: New York (Micropaleontology Press), pp. 167-198.

CLIMAP Project Members, 1981. Seasonal reconstruction of the earth's surface at the last glacial maximum. Geol. Soc. Am. Map and Chart Series MC-36.

Hulsemann, J., 1966. Notes: on the routine analysis of carbonate in unconsolidated sediments. J. Sed. Petrol., 36:622-625.

Imbrie, J., Hays, J. D., Martinson, D. G., McIntyre, A., Mix, A. C., Morley, J. J., Pisias, N. G., Prell, W. L., and Shackleton, N. J., 1984. The orbital theory of Pleistocene climate: support from a revised chronology of the marine ${ }^{18} \mathrm{O}$ record. In Berger, A., Hays, J., Imbrie, J., et al. (Eds.), Milankovitch and Climate (Vol. II): Boston (D. Reidel Pub. Co.), 269-305.

Kent, D., Opdyke, N. D., and Ewing, M., 1971. Climate change in the North Pacific using ice-rafted detritus as a climate indicator. Geol. Soc. Am. Bull., 82:2741-2754.

Kipp, N. D., 1976. New transfer function for estimating past sea-surface conditions from sea-bed distribution of planktonic foraminiferal assemblages in the North Atlantic. In Cline, R. M., and Hays, J. D. (Eds.), Investigation of Late Quaternary Paleoceanography and Paleoclimatology. Geol. Soc. Am. Mem., 145:3-42.

Manabe, S., and Broccoli, A. J., 1985. The influence of continental ice sheets on the climate of an ice age. J. Geophys. Res., 90:21672190.

Ruddiman, W. F., 1971. Pleistocene sedimentation in the equatorial Atlantic: stratigraphy and faunal paleoclimatology. Geol. Soc. Am. Bull., 82:283-301.

1972. Sediment redistribution on the eastern Reykjanes Ridge flank: seismic evidence. Geol. Soc. Am. Bull., 83:2039-2062.

1977. Late Quaternary deposition of ice-rafted sand in the Subpolar North Atlantic (lat. $40^{\circ}$ to $65^{\circ} \mathrm{N}$ ). Geol. Soc. Am. Bull., 88:1813-1827.

Ruddiman, W. F., and McIntyre, A., 1976. Northeast Atlantic paleoclimatic changes over the past 600,000 years. In Cline, R. M., and Hays, J. D. (Eds.), Investigation of Late Quaternary Paleoceanography and Paleoclimatology, Geol. Soc. Am. Mem., 145:111-146. 1981. Oceanic mechanisms for amplification of the 23,000year ice volume cycle. Science, 212:617-627.

1984. Ice-age thermal response and climatic role of the surface Atlantic Ocean, $40^{\circ}$ to $63^{\circ}$ N. Geol. Soc. Am. Bull., 95:381396.

Ruddiman, W. F., Raymo, M., and McIntyre, A., in press. Matuyama 41,000-year cycles: North Atlantic Ocean and Northern Hemisphere ice sheets. Earth Planet. Sci. Lett.

Ruddiman, W. F., Shackleton, N. J., and Mclntyre, A., in press. North Atlantic Sea-Surface Temperatures for the Last 1.1 m.y. In Summerhayes, C. P., and Shackleton, N. J. (Eds.), Geol. Soc. London Spec. Publ.

Shackleton, N. J., 1977. The oxygen isotope stratigraphic record of the Late Pleistocene. R. Soc. London Phil. Trans. B, 280:169-178.

Shackleton, N. J., Backman, J., Zimmerman, H., Kent, D. V., Hall, M. A., Roberts, D. G., Schnitker, D., Baldauf, J. G., Desprairies, A., Homrighausen, R., Huddlestun, P., Keene, J. B., Kaltenback, A. J., Krumsiek, K. A. O., Morton, A. C., Murray, J. W., and Westberg-Smith, J., 1984. Oxygen isotope calibration of the onset of ice rafting in DSDP Site 552A: history of glaciation in the North Atlantic region. Nature (London), 307:620-623.

Shackleton, N. J., and Opdyke, N. D., 1973. Oxygen isotope and paleomagnetic stratigraphy of equatorial Pacific core V28-238: oxygen isotope temperatures and ice volumes on a $10^{5}$ and $10^{6}$ year scale. Quat. Res., 3:39-55.

Zimmerman, H. B., Shackleton, N. J., Backman, J., Kent, D. V., Baldauf, J. G., Kaltenback, A. J., and Morton, A. C., 1985. History of Plio-Pleistocene Climate in the Northeastern Atlantic, Deep Sea Drilling Project Hole 552A. In Roberts, D. G., Schnitker, D., et al., Init. Repts. DSDP, 81: Washington (U.S. Govt. Printing Office), 861-876.

Date of Initial Receipt: 18 December 1984

Date of Acceptance: 25 October 1985 
PALEOCEANOGRAPHY FROM NORTH ATLANTIC SITES 607 AND 609

APPENDIX A

DSDP Site 609: Calcium Carbonate Values

\begin{tabular}{|c|c|c|c|c|c|c|c|c|c|}
\hline $\begin{array}{l}\text { Sub-bottom } \\
\text { depth } \\
\text { (m) }\end{array}$ & $\begin{array}{c}\mathrm{CaCO}_{3} \\
(\%)\end{array}$ & $\begin{array}{l}\text { Sub-bottom } \\
\text { depth } \\
\text { (m) }\end{array}$ & $\begin{array}{c}\mathrm{CaCO}_{3} \\
(\%)\end{array}$ & $\begin{array}{l}\text { Sub-bottom } \\
\text { depth } \\
\text { (m) }\end{array}$ & $\begin{array}{c}\mathrm{CaCO}_{3} \\
(\%)\end{array}$ & $\begin{array}{l}\text { Sub-bottom } \\
\text { depth } \\
\text { (m) }\end{array}$ & $\begin{array}{c}\mathrm{CaCO}_{3} \\
(\%)\end{array}$ & $\begin{array}{l}\text { Sub-bottom } \\
\text { depth } \\
\text { (m) }\end{array}$ & $\begin{array}{c}\mathrm{CaCO}_{3} \\
(\%)\end{array}$ \\
\hline 0.06 & 79.10 & 21.11 & 85.80 & 42.11 & 71.50 & 66.41 & 58.58 & 90.41 & 79.59 \\
\hline 0.31 & 71.44 & 21.41 & 84.27 & 42.41 & 29.33 & 66.71 & 64.58 & 90.71 & 71.09 \\
\hline 0.61 & 40.62 & 21.71 & 81.77 & 42.71 & 15.08 & 67.01 & 61.54 & 91.01 & 69.35 \\
\hline 0.91 & 32.70 & 22.01 & 80.15 & 43.01 & 67.39 & 67.31 & 50.74 & 91.31 & 72.85 \\
\hline 1.21 & 22.70 & 22.31 & 15.43 & 43.31 & 77.69 & 67.61 & 7.15 & 91.61 & 67.32 \\
\hline 1.41 & 10.54 & 22.61 & 31.59 & 43.61 & 56.51 & 67.91 & 18.03 & 91.91 & 60.79 \\
\hline 1.81 & 16.24 & 22.91 & 33.37 & 43.91 & 16.92 & 68.21 & 38.96 & 92.21 & 38.48 \\
\hline 2.11 & 28.98 & 23.06 & 13.97 & 44.21 & 21.95 & 68.51 & 62.77 & 92.51 & 31.40 \\
\hline 2.41 & 23.18 & 23.21 & 12.63 & 45.41 & 51.83 & 68.81 & 45.80 & 95.21 & 76.59 \\
\hline 2.71 & 35.49 & 23.35 & 17.73 & 45.71 & 76.73 & 69.11 & 49.17 & 95.51 & 74.09 \\
\hline 3.01 & 31.43 & 23.51 & 37.46 & 46.01 & 62.47 & 69.41 & 55.86 & 95.81 & 72.56 \\
\hline 3.31 & 56.11 & 23.65 & 28.05 & 46.31 & 57.71 & 69.71 & 49.71 & 96.11 & 41.99 \\
\hline 3.61 & 43.10 & 23.81 & 46.37 & 46.61 & 63.90 & 70.01 & 47.65 & 96.41 & 21.69 \\
\hline 3.91 & 19.80 & 23.95 & 11.91 & 46.91 & 64.97 & 70.31 & 17.83 & 96.71 & 50.28 \\
\hline 4.21 & 37.11 & 24.11 & 16.62 & 47.21 & 55.49 & 70.61 & 9.97 & 97.01 & 49.47 \\
\hline 4.51 & 64.23 & 24.25 & 45.90 & 47.51 & 76.41 & 70.91 & 35.19 & 97.31 & 45.87 \\
\hline 4.81 & 78.85 & 24.41 & 46.64 & 47.81 & 63.65 & 71.21 & 40.06 & 97.61 & 69.16 \\
\hline 5.11 & 54.76 & 24.56 & 31.51 & 48.11 & 39.81 & 71.51 & 13.72 & 97.91 & 72.56 \\
\hline 5.41 & 76.13 & 24.71 & 17.77 & 48.41 & 28.91 & 71.81 & 35.65 & 98.21 & 70.31 \\
\hline 5.71 & 79.26 & 24.85 & 24.27 & 48.71 & 18.73 & 72.11 & 42.01 & 98.51 & 66.97 \\
\hline 6.01 & 66.23 & 25.01 & 51.13 & 49.01 & 11.16 & 72.41 & 56.64 & 98.81 & 71.83 \\
\hline 6.31 & 61.15 & 25.15 & 70.71 & 49.31 & 5.96 & 72.71 & 63.39 & 99.11 & 66.70 \\
\hline 6.61 & 66.85 & 25.31 & 58.94 & 49.61 & 6.20 & 73.01 & 72.22 & 99.41 & 74.81 \\
\hline 6.79 & 78.61 & 25.45 & 84.09 & 49.91 & 10.05 & 73.31 & 69.63 & 99.71 & 36.50 \\
\hline 7.31 & 15.83 & 26.51 & 84.05 & 50.21 & 35.32 & 73.61 & 53.52 & 100.01 & 32.85 \\
\hline 7.61 & 36.55 & 26.81 & 85.78 & 50.51 & 51.90 & 74.51 & 71.75 & 100.31 & 67.81 \\
\hline 7.91 & 58.02 & 27.11 & 83.54 & 50.81 & 56.47 & 74.81 & 56.92 & 100.61 & 58.41 \\
\hline 8.21 & 28.38 & 27.41 & 87.47 & 51.11 & 45.47 & 75.11 & 39.81 & 100.91 & 80.37 \\
\hline 8.51 & 34.66 & 27.71 & 73.27 & 51.41 & 50.49 & 75.41 & 24.32 & 101.14 & 51.44 \\
\hline 8.81 & 47.82 & 28.01 & 70.87 & 51.71 & 11.36 & 75.71 & 57.08 & 103.11 & 66.16 \\
\hline 9.11 & 64.63 & 28.31 & 80.66 & 52.01 & 33.75 & 76.01 & 27.95 & 103.31 & 58.43 \\
\hline 9.41 & 72.11 & 28.61 & 83.65 & 52.31 & 24.23 & 76.31 & 42.54 & 103.61 & 59.89 \\
\hline 9.71 & 66.64 & 28.91 & 79.42 & 52.61 & 8.15 & 76.61 & 27.50 & 103.91 & 59.24 \\
\hline 10.01 & 75.14 & 29.21 & 34.39 & 52.91 & 48.42 & 76.91 & 48.29 & 104.21 & 69.02 \\
\hline 10.31 & 60.95 & 29.51 & 56.19 & 53.21 & 55.75 & 77.21 & 77.01 & 104.51 & 68.49 \\
\hline 10.61 & 12.94 & 29.81 & 57.53 & 53.51 & 74.49 & 77.51 & 74.80 & 104.81 & 33.50 \\
\hline 10.91 & 35.53 & 30.11 & 44.88 & 53.81 & 78.53 & 77.81 & 68.96 & 105.11 & 85.64 \\
\hline 11.21 & 68.84 & 30.41 & 70.58 & 54.11 & 75.50 & 78.11 & 45.31 & 105.41 & 84.94 \\
\hline 11.51 & 80.13 & 30.71 & 73.60 & 54.41 & 61.45 & 78.41 & 22.94 & 105.71 & 71.53 \\
\hline 11.81 & 43.21 & 31.01 & 40.51 & 54.71 & 45.04 & 78.71 & 28.87 & 106.01 & 76.16 \\
\hline 12.11 & 23.59 & 31.31 & 63.41 & 54.91 & 34.04 & 79.01 & 31.58 & 106.31 & 63.19 \\
\hline 12.41 & 39.40 & 31.61 & 56.08 & 55.31 & 54.27 & 79.31 & 18.74 & 106.61 & 72.22 \\
\hline 12.71 & 23.61 & 31.91 & 65.04 & 55.61 & 60.02 & 79.61 & 45.27 & 106.91 & 65.02 \\
\hline 13.01 & 51.98 & 32.21 & 65.92 & 55.91 & 62.00 & 79.91 & 82.60 & 107.21 & 47.33 \\
\hline 13.31 & 38.65 & 32.51 & 64.92 & 56.21 & 56.01 & 80.21 & 70.47 & 107.51 & 65.50 \\
\hline 13.61 & 42.99 & 32.81 & 72.65 & 56.51 & 59.10 & 80.51 & 75.25 & 107.81 & 71.60 \\
\hline 13.91 & 17.08 & 33.11 & 51.49 & 56.81 & 38.88 & 80.53 & 71.50 & 108.11 & 85.85 \\
\hline 14.21 & 13.14 & 33.41 & 39.89 & 57.11 & 41.94 & 80.81 & 23.15 & 108.41 & 69.89 \\
\hline 14.51 & 67.69 & 33.71 & 15.62 & 57.41 & 15.36 & 81.11 & 34.21 & 108.71 & 73.95 \\
\hline 14.81 & 77.39 & 34.01 & 18.57 & 57.71 & 70.49 & 81.41 & 19.45 & 109.01 & 76.69 \\
\hline 15.11 & 58.88 & 34.21 & $50.81 \mathrm{~B}$ & 58.01 & 71.51 & 81.71 & 22.74 & 109.31 & 70.88 \\
\hline 15.41 & 53.77 & 34.51 & $38.94 \mathrm{~B}$ & 58.31 & 53.71 & 82.01 & 62.98 & 109.61 & 73.59 \\
\hline 15.71 & 78.52 & 34.81 & $18.28 \mathrm{~B}$ & 58.61 & 23.12 & 82.31 & 45.98 & 109.91 & 50.78 \\
\hline 16.01 & 54.29 & 35.11 & $31.75 \mathrm{~B}$ & 58.91 & 59.22 & 82.61 & 72.81 & 110.21 & 30.93 \\
\hline 16.31 & 64.67 & 35.41 & $30.85 \mathrm{~B}$ & 59.21 & 15.55 & 82.91 & 67.43 & 110.51 & 54.80 \\
\hline 17.51 & 80.06 & 35.71 & $20.12 \mathrm{~B}$ & 59.51 & 58.66 & 84.11 & 51.46 & 110.81 & 52.29 \\
\hline 17.81 & 78.33 & 36.01 & $27.46 \mathrm{~B}$ & 59.81 & 48.32 & 84.41 & 58.13 & 111.11 & 52.16 \\
\hline 17.95 & 77.73 & 36.31 & $29.42 \mathrm{~B}$ & 60.11 & 75.98 & 84.71 & 62.26 & 114.71 & 28.95 \\
\hline 18.11 & 13.94 & 36.61 & $30.32 \mathrm{~B}$ & 60.41 & 58.07 & 85.01 & 62.13 & 115.01 & 44.86 \\
\hline 18.25 & 8.13 & 36.91 & $29.83 \mathrm{~B}$ & 60.71 & 62.94 & 85.31 & 48.12 & 115.31 & 36.57 \\
\hline 18.41 & 27.43 & 37.21 & $47.16 \mathrm{~B}$ & 61.01 & 55.16 & 85.61 & 23.39 & 115.61 & 58.24 \\
\hline 18.56 & 21.09 & 37.51 & $16.72 \mathrm{~B}$ & 61.31 & 64.40 & 85.91 & 64.36 & 115.91 & 79.02 \\
\hline 18.71 & 13.13 & 37.81 & $63.11 \mathrm{~B}$ & 61.61 & 48.16 & 86.21 & 88.79 & 116.21 & 27.14 \\
\hline 18.85 & 22.19 & 38.11 & $77.93 \mathrm{~B}$ & 61.91 & 61.78 & 86.51 & 58.11 & 116.51 & 37.98 \\
\hline 19.01 & 14.26 & 38.41 & $67.80 \mathrm{~B}$ & 62.21 & 59.59 & 86.81 & 61.41 & 116.81 & 48.84 \\
\hline 19.15 & 53.40 & 38.81 & 71.03 & 62.51 & 25.92 & 87.11 & 61.07 & 117.11 & 38.22 \\
\hline 19.31 & 57.25 & 39.11 & 59.76 & 62.81 & 32.38 & 87.41 & 58.97 & 117.41 & 65.42 \\
\hline 19.45 & 6.47 & 39.41 & 67.34 & 63.11 & 72.18 & 87.71 & 54.95 & 117.71 & 67.39 \\
\hline 19.61 & 69.46 & 39.71 & 63.86 & 63.41 & 86.25 & 88.01 & 52.51 & 118.01 & 62.94 \\
\hline 19.75 & 79.29 & 40.01 & 51.85 & 63.71 & 81.11 & 88.31 & 59.52 & 118.31 & 62.92 \\
\hline 19.91 & 60.29 & 40.31 & 3.81 & 64.01 & 61.66 & 88.61 & 52.64 & 118.61 & 58.47 \\
\hline 20.06 & 46.17 & 40.61 & 4.58 & 64.91 & 47.11 & 88.91 & 39.97 & 118.91 & 60.56 \\
\hline 20.21 & 72.38 & 40.91 & 36.55 & 65.21 & 59.42 & 89.21 & 55.37 & 119.21 & 69.69 \\
\hline 20.35 & 74.80 & 41.21 & 57.36 & 65.51 & 60.30 & 89.51 & 49.44 & 119.51 & 66.90 \\
\hline 20.51 & 80.96 & 41.51 & 41.98 & 65.81 & 62.90 & 89.81 & 71.25 & 119.81 & 68.65 \\
\hline 20.81 & 85.54 & 41.81 & 70.47 & 66.11 & 62.38 & 90.11 & 74.13 & 120.11 & 67.61 \\
\hline
\end{tabular}


Appendix A (continued).

\begin{tabular}{|c|c|c|c|c|c|c|c|c|c|}
\hline $\begin{array}{l}\text { Sub-bottom } \\
\text { depth } \\
\text { (m) }\end{array}$ & $\underset{(\%)}{\mathrm{CaCO}_{3}}$ & $\begin{array}{l}\text { Sub-bottom } \\
\text { depth } \\
\text { (m) }\end{array}$ & $\underset{(\%)}{\mathrm{CaCO}_{3}}$ & $\begin{array}{l}\text { Sub-bottom } \\
\text { depth } \\
\text { (m) }\end{array}$ & $\begin{array}{c}\mathrm{CaCO}_{3} \\
(\%)\end{array}$ & $\begin{array}{l}\text { Sub-bottom } \\
\text { depth } \\
\text { (m) }\end{array}$ & $\begin{array}{c}\mathrm{CaCO}_{3} \\
(\%)\end{array}$ & $\begin{array}{l}\text { Sub-bottom } \\
\text { depth } \\
\text { (m) }\end{array}$ & $\begin{array}{c}\mathrm{CaCO}_{3} \\
(\%)\end{array}$ \\
\hline 120.41 & 30.95 & 144.41 & 66.09 & 169.01 & $67.29 \mathrm{~B}$ & 194.81 & $75.02 \mathrm{~B}$ & 217.61 & $81.24 \mathrm{~B}$ \\
\hline 120.71 & 39.75 & 144.71 & 73.70 & 169.31 & $31.60 \mathrm{~B}$ & 195.11 & $75.43 \mathrm{~B}$ & 217.91 & $78.70 \mathrm{~B}$ \\
\hline 121.01 & 50.65 & 145.01 & 82.45 & 169.61 & $75.93 \mathrm{~B}$ & 195.41 & $72.29 \mathrm{~B}$ & 218.21 & $78.30 \mathrm{~B}$ \\
\hline 122.25 & 71.22 & 145.31 & 41.02 & 169.91 & $73.73 \mathrm{~B}$ & 195.71 & $73.03 \mathrm{~B}$ & 218.51 & $77.40 \mathrm{~B}$ \\
\hline 122.51 & 66.13 & 145.61 & 50.31 & 170.21 & 67.91 B & 196.01 & $74.76 \mathrm{~B}$ & 218.81 & $89.20 \mathrm{~B}$ \\
\hline 122.81 & 47.08 & 145.91 & 77.72 & 170.51 & $66.94 \mathrm{~B}$ & 196.31 & $81.34 \mathrm{~B}$ & 219.11 & $78.09 \mathrm{~B}$ \\
\hline 123.11 & 48.74 & 146.21 & 83.27 & 170.81 & $66.85 \mathrm{~B}$ & 196.61 & $82.77 \mathrm{~B}$ & 219.41 & $76.11 \mathrm{~B}$ \\
\hline 123.41 & 65.66 & 146.51 & 78.37 & 171.11 & $61.52 \mathrm{~B}$ & 196.91 & $75.76 \mathrm{~B}$ & 219.71 & $69.11 \mathrm{~B}$ \\
\hline 123.71 & 84.00 & 146.81 & 64.03 & 171.41 & $43.79 \mathrm{~B}$ & 197.21 & $67.65 \mathrm{~B}$ & 219.96 & $78.03 \mathrm{~B}$ \\
\hline 124.01 & 67.37 & 147.11 & 60.19 & 171.71 & $52.26 \mathrm{~B}$ & 197.51 & $63.80 \mathrm{~B}$ & 220.31 & $85.09 \mathrm{~B}$ \\
\hline 124.31 & 74.89 & 147.41 & 70.25 & 172.01 & $80.84 \mathrm{~B}$ & 197.81 & $64.53 \mathrm{~B}$ & 220.61 & $80.57 \mathrm{~B}$ \\
\hline 124.61 & 50.69 & 147.71 & 50.02 & 172.19 & $77.44 \mathrm{~B}$ & 198.11 & $71.42 \mathrm{~B}$ & 220.91 & $83.27 \mathrm{~B}$ \\
\hline 124.91 & 49.27 & 148.01 & 31.02 & 172.31 & $52.33 \mathrm{~B}$ & 198.41 & $71.70 \mathrm{~B}$ & 221.21 & $84.09 \mathrm{~B}$ \\
\hline 125.21 & 67.33 & 148.31 & 30.22 & 172.61 & $63.67 \mathrm{~B}$ & 198.71 & $69.12 \mathrm{~B}$ & 221.51 & $80.84 \mathrm{~B}$ \\
\hline 125.51 & 74.91 & 148.61 & 41.22 & 172.91 & $61.15 \mathrm{~B}$ & 199.01 & $67.33 \mathrm{~B}$ & 221.81 & $78.01 \mathrm{~B}$ \\
\hline 125.81 & 69.78 & 148.91 & 80.83 & 173.21 & $53.16 \mathrm{~B}$ & 199.31 & $71.68 \mathrm{~B}$ & 222.11 & $81.43 \mathrm{~B}$ \\
\hline 126.11 & 66.10 & 149.21 & 84.54 & 173.51 & $60.54 \mathrm{~B}$ & 199.61 & $71.37 \mathrm{~B}$ & 222.41 & 84.93 B \\
\hline 126.41 & 68.57 & 150.11 & 70.22 & 173.81 & $67.36 \mathrm{~B}$ & 199.91 & $72.79 \mathrm{~B}$ & 222.71 & $81.81 \mathrm{~B}$ \\
\hline 126.71 & 68.06 & 150.41 & 69.61 & 174.11 & $72.02 \mathrm{~B}$ & 200.21 & $66.57 \mathrm{~B}$ & 223.01 & $80.47 \mathrm{~B}$ \\
\hline 127.01 & 62.34 & 150.71 & 68.38 & 174.41 & $67.62 \mathrm{~B}$ & 200.51 & $62.50 \mathrm{~B}$ & 223.31 & $77.45 \mathrm{~B}$ \\
\hline 127.31 & 64.23 & 151.01 & 75.03 & 174.71 & $65.44 \mathrm{~B}$ & 200.81 & $68.58 \mathrm{~B}$ & 223.61 & $75.03 \mathrm{~B}$ \\
\hline 127.61 & 65.36 & 151.31 & 67.11 & 175.01 & $70.70 \mathrm{~B}$ & 201.11 & $71.58 \mathrm{~B}$ & 223.91 & $73.74 \mathrm{~B}$ \\
\hline 127.91 & 67.61 & 151.61 & 49.50 & 175.31 & $68.50 \mathrm{~B}$ & 201.41 & $76.61 \mathrm{~B}$ & 224.21 & $75.12 \mathrm{~B}$ \\
\hline 128.21 & 66.22 & 151.91 & 20.06 & 175.61 & $67.45 \mathrm{~B}$ & 201.71 & $84.67 \mathrm{~B}$ & 224.51 & $75.51 \mathrm{~B}$ \\
\hline 128.51 & 62.76 & 152.19 & 51.61 & 175.91 & $72.66 \mathrm{~B}$ & 202.01 & $80.09 \mathrm{~B}$ & 224.81 & $78.69 \mathrm{~B}$ \\
\hline 128.81 & 68.58 & 152.21 & 47.67 & 176.21 & $50.47 \mathrm{~B}$ & 202.31 & $72.98 \mathrm{~B}$ & 225.11 & $82.14 \mathrm{~B}$ \\
\hline 129.11 & 51.28 & 152.51 & 24.47 & 176.51 & $44.98 \mathrm{~B}$ & 202.61 & $69.59 \mathrm{~B}$ & 225.41 & $79.41 \mathrm{~B}$ \\
\hline 129.41 & 26.00 & 152.81 & 81.74 & 176.81 & $70.65 \mathrm{~B}$ & 202.91 & $71.48 \mathrm{~B}$ & 225.71 & $81.61 \mathrm{~B}$ \\
\hline 129.71 & 84.68 & 153.11 & 72.30 & 177.11 & $67.58 \mathrm{~B}$ & 203.21 & $70.65 \mathrm{~B}$ & 226.01 & $80.19 \mathrm{~B}$ \\
\hline 130.01 & 83.68 & 153.41 & 68.68 & 177.41 & $70.03 \mathrm{~B}$ & 203.51 & $74.29 \mathrm{~B}$ & 226.31 & $80.08 \mathrm{~B}$ \\
\hline 130.31 & 75.37 & 153.71 & 67.91 & 177.71 & $59.61 \mathrm{~B}$ & 203.81 & $76.82 \mathrm{~B}$ & 226.61 & $76.65 \mathrm{~B}$ \\
\hline 130.61 & 33.53 & 154.01 & 58.44 & 178.01 & $75.33 \mathrm{~B}$ & 204.11 & $79.48 \mathrm{~B}$ & 226.91 & $75.80 \mathrm{~B}$ \\
\hline 130.91 & 83.54 & 154.31 & 59.83 & 178.31 & $72.22 \mathrm{~B}$ & 204.41 & $78.15 \mathrm{~B}$ & 227.21 & $78.21 \mathrm{~B}$ \\
\hline 131.21 & 84.47 & 154.61 & 50.64 & 181.91 & $75.94 \mathrm{~B}$ & 204.71 & $79.28 \mathrm{~B}$ & 227.51 & $81.93 \mathrm{~B}$ \\
\hline 131.47 & 77.67 & 154.91 & 59.10 & 182.21 & $78.55 \mathrm{~B}$ & 205.01 & $74.30 \mathrm{~B}$ & 227.81 & $82.51 \mathrm{~B}$ \\
\hline 131.81 & 68.99 & 155.21 & 56.94 & 182.51 & $72.98 \mathrm{~B}$ & 205.31 & $65.03 \mathrm{~B}$ & 228.11 & $82.41 \mathrm{~B}$ \\
\hline 132.11 & 70.73 & 155.51 & 61.15 & 182.81 & $73.37 \mathrm{~B}$ & 205.61 & $71.65 \mathrm{~B}$ & 228.41 & $81.68 \mathrm{~B}$ \\
\hline 132.41 & 72.88 & 155.81 & 47.89 & 183.11 & $68.78 \mathrm{~B}$ & 205.91 & $70.73 \mathrm{~B}$ & 228.71 & $82.70 \mathrm{~B}$ \\
\hline 132.71 & 60.81 & 156.11 & 70.34 & 183.41 & $75.83 \mathrm{~B}$ & 206.21 & $68.31 \mathrm{~B}$ & 229.01 & $83.16 \mathrm{~B}$ \\
\hline 133.01 & 46.92 & 156.41 & 70.98 & 183.71 & $66.88 \mathrm{~B}$ & 206.51 & $67.17 \mathrm{~B}$ & 229.31 & $80.58 \mathrm{~B}$ \\
\hline 133.31 & 67.76 & 156.71 & 75.58 & 184.01 & $58.39 \mathrm{~B}$ & 206.81 & $62.99 \mathrm{~B}$ & 229.61 & $81.73 \mathrm{~B}$ \\
\hline 133.61 & 57.29 & 157.01 & 70.16 & 184.31 & $58.68 \mathrm{~B}$ & 207.11 & $74.76 \mathrm{~B}$ & 229.83 & $75.71 \mathrm{~B}$ \\
\hline 133.91 & 48.08 & 157.31 & 68.14 & 184.61 & $57.69 \mathrm{~B}$ & 207.41 & $76.23 \mathrm{~B}$ & 229.98 & $82.77 \mathrm{~B}$ \\
\hline 134.21 & 45.85 & 157.61 & 65.59 & 184.91 & $63.30 \mathrm{~B}$ & 207.71 & $71.72 \mathrm{~B}$ & 230.21 & $78.50 \mathrm{~B}$ \\
\hline 134.51 & 48.20 & 157.91 & 63.84 & 185.21 & $63.03 \mathrm{~B}$ & 208.01 & $65.35 \mathrm{~B}$ & 230.51 & $82.03 \mathrm{~B}$ \\
\hline 134.81 & 14.80 & 158.21 & 59.58 & 185.51 & $69.60 \mathrm{~B}$ & 208.31 & $64.67 \mathrm{~B}$ & 230.81 & $82.31 \mathrm{~B}$ \\
\hline 135.11 & 75.85 & 158.51 & 61.76 & 185.81 & $70.18 \mathrm{~B}$ & 208.61 & $70.88 \mathrm{~B}$ & 231.11 & $77.07 \mathrm{~B}$ \\
\hline 135.41 & 51.27 & 158.81 & 70.38 & 186.11 & $70.16 \mathrm{~B}$ & 208.91 & $70.35 \mathrm{~B}$ & 231.48 & $80.46 \mathrm{~B}$ \\
\hline 135.71 & 83.17 & 159.11 & 66.16 & 186.41 & $68.07 \mathrm{~B}$ & 209.21 & $67.21 \mathrm{~B}$ & 231.71 & $77.38 \mathrm{~B}$ \\
\hline 136.01 & 80.32 & 159.71 & 64.60 & 186.71 & $67.28 \mathrm{~B}$ & 209.51 & 70.65 B & 232.01 & $79.79 \mathrm{~B}$ \\
\hline 136.31 & 78.10 & 160.01 & 60.45 & 187.01 & $72.62 \mathrm{~B}$ & 209.81 & $74.46 \mathrm{~B}$ & 232.31 & 85.32 B \\
\hline 136.61 & 67.88 & 160.31 & 60.39 & 187.31 & $71.52 \mathrm{~B}$ & 210.11 & $79.24 \mathrm{~B}$ & 232.61 & $85.17 \mathrm{~B}$ \\
\hline 136.91 & 79.28 & 160.61 & 63.82 & 187.61 & $71.93 \mathrm{~B}$ & 210.41 & $73.04 \mathrm{~B}$ & 232.91 & $85.43 \mathrm{~B}$ \\
\hline 137.21 & 12.37 & 160.95 & 67.65 & 187.91 & $72.33 \mathrm{~B}$ & 210.64 & $77.05 \mathrm{~B}$ & 233.21 & $82.81 \mathrm{~B}$ \\
\hline 137.51 & 76.75 & 161.21 & 69.13 & 188.21 & $69.64 \mathrm{~B}$ & 210.79 & $75.48 \mathrm{~B}$ & 233.51 & $83.41 \mathrm{~B}$ \\
\hline 137.81 & 74.82 & 161.36 & 70.12 & 188.51 & $71.52 \mathrm{~B}$ & 211.01 & $73.60 \mathrm{~B}$ & 233.81 & $84.85 \mathrm{~B}$ \\
\hline 138.11 & 72.38 & 162.71 & $70.35 \mathrm{~B}$ & 188.81 & $73.28 \mathrm{~B}$ & 211.31 & $69.31 \mathrm{~B}$ & 234.11 & $80.87 \mathrm{~B}$ \\
\hline 138.41 & 53.43 & 163.01 & $68.40 \mathrm{~B}$ & 189.11 & $70.82 \mathrm{~B}$ & 211.61 & $71.43 \mathrm{~B}$ & 234.41 & $81.38 \mathrm{~B}$ \\
\hline 138.71 & 70.39 & 163.31 & $60.70 \mathrm{~B}$ & 189.41 & $68.35 \mathrm{~B}$ & 211.91 & $65.44 \mathrm{~B}$ & 234.71 & $82.37 \mathrm{~B}$ \\
\hline 139.01 & 77.98 & 163.61 & $64.44 \mathrm{~B}$ & 189.71 & $68.55 \mathrm{~B}$ & 212.21 & $65.87 \mathrm{~B}$ & 235.01 & $75.93 \mathrm{~B}$ \\
\hline 139.31 & 74.45 & 163.91 & $75.56 \mathrm{~B}$ & 190.01 & $69.25 \mathrm{~B}$ & 212.51 & $64.63 \mathrm{~B}$ & 235.31 & $83.61 \mathrm{~B}$ \\
\hline 139.61 & 69.48 & 164.21 & $73.96 \mathrm{~B}$ & 190.31 & $71.29 \mathrm{~B}$ & 212.81 & $76.13 \mathrm{~B}$ & 235.61 & 89.92 В \\
\hline 139.91 & 64.11 & 164.51 & $69.42 \mathrm{~B}$ & 190.61 & $70.60 \mathrm{~B}$ & 213.11 & $85.73 \mathrm{~B}$ & 235.91 & $89.25 \mathrm{~B}$ \\
\hline 140.25 & 74.83 & 164.81 & $68.91 \mathrm{~B}$ & 190.91 & $74.67 \mathrm{~B}$ & 213.41 & 84.44 B & 236.21 & $90.27 \mathrm{~B}$ \\
\hline 140.51 & 68.72 & 165.11 & $64.59 \mathrm{~B}$ & 191.21 & $76.47 \mathrm{~B}$ & 213.71 & $66.57 \mathrm{~B}$ & 236.51 & 88.92 B \\
\hline 140.81 & 71.96 & 165.41 & $57.02 \mathrm{~B}$ & 191.44 & $78.46 \mathrm{~B}$ & 214.01 & $82.04 \mathrm{~B}$ & 236.81 & $89.56 \mathrm{~B}$ \\
\hline 141.11 & 70.44 & 165.71 & $56.63 \mathrm{~B}$ & 191.51 & $68.66 \mathrm{~B}$ & 214.31 & $82.93 \mathrm{~B}$ & 237.11 & 88.93 B \\
\hline 141.41 & 73.69 & 166.01 & $49.84 \mathrm{~B}$ & 191.81 & $69.42 \mathrm{~B}$ & 214.61 & 81.62 B & 237.41 & $95.42 \mathrm{~B}$ \\
\hline 141.71 & 73.01 & 166.31 & $49.59 \mathrm{~B}$ & 192.11 & $66.22 \mathrm{~B}$ & 214.91 & $79.04 \mathrm{~B}$ & 237.71 & 89.17 B \\
\hline 142.01 & 66.80 & 166.61 & $53.00 \mathrm{~B}$ & 192.41 & $60.91 \mathrm{~B}$ & 215.21 & $78.09 \mathrm{~B}$ & 238.01 & $92.13 \mathrm{~B}$ \\
\hline 142.31 & 77.01 & 166.91 & $60.48 \mathrm{~B}$ & 192.71 & $60.28 \mathrm{~B}$ & 215.51 & $76.32 \mathrm{~B}$ & 238.31 & 89.93 B \\
\hline 142.61 & 51.97 & 167.21 & $59.31 \mathrm{~B}$ & 193.01 & $63.48 \mathrm{~B}$ & 215.81 & $79.10 \mathrm{~B}$ & 238.61 & $88.80 \mathrm{~B}$ \\
\hline 142.91 & 50.55 & 167.51 & $63.58 \mathrm{~B}$ & 193.31 & $71.04 \mathrm{~B}$ & 216.11 & $77.89 \mathrm{~B}$ & 238.91 & $85.80 \mathrm{~B}$ \\
\hline 143.21 & 41.06 & 167.81 & $69.85 \mathrm{~B}$ & 193.61 & $72.22 \mathrm{~B}$ & 216.41 & $77.85 \mathrm{~B}$ & 239.21 & $87.19 \mathrm{~B}$ \\
\hline 143.51 & 45.10 & 168.11 & $70.41 \mathrm{~B}$ & 193.91 & $74.26 \mathrm{~B}$ & 216.71 & $76.02 \mathrm{~B}$ & 239.51 & $91.55 \mathrm{~B}$ \\
\hline 143.81 & 61.40 & 168.41 & $63.21 \mathrm{~B}$ & 194.21 & $76.32 \mathrm{~B}$ & 217.01 & $75.54 \mathrm{~B}$ & 239.81 & $92.23 \mathrm{~B}$ \\
\hline 144.12 & 87.79 & 168.71 & $67.41 \mathrm{~B}$ & 194.51 & $73.04 \mathrm{~B}$ & 217.31 & $79.00 \mathrm{~B}$ & 240.11 & $89.83 \mathrm{~B}$ \\
\hline
\end{tabular}


Appendix A (continued).

\begin{tabular}{|c|c|c|c|c|c|c|c|c|c|}
\hline $\begin{array}{l}\text { Sub-bottom } \\
\text { depth } \\
\text { (m) }\end{array}$ & $\begin{array}{c}\mathrm{CaCO}_{3} \\
(\%)\end{array}$ & $\begin{array}{l}\text { Sub-bottom } \\
\text { depth } \\
\text { (m) }\end{array}$ & $\begin{array}{c}\mathrm{CaCO}_{3} \\
(\%)\end{array}$ & $\begin{array}{l}\text { Sub-bottom } \\
\text { depth } \\
\text { (m) }\end{array}$ & $\begin{array}{c}\mathrm{CaCO}_{3} \\
(\%)\end{array}$ & $\begin{array}{l}\text { Sub-bottom } \\
\text { depth } \\
\text { (m) }\end{array}$ & $\begin{array}{c}\mathrm{CaCO}_{3} \\
(\%)\end{array}$ & $\begin{array}{l}\text { Sub-bottom } \\
\text { depth } \\
\text { (m) }\end{array}$ & $\begin{array}{c}\mathrm{CaCO}_{3} \\
(\%)\end{array}$ \\
\hline 240.41 & $90.37 \mathrm{~B}$ & 246.11 & $90.58 \mathrm{~B}$ & 251.49 & $93.18 \mathrm{~B}$ & 257.21 & 89.75 B & 263.51 & $91.87 \mathrm{~B}$ \\
\hline 240.71 & $92.61 \mathrm{~B}$ & 246.41 & 89.85 B & 251.81 & $94.10 \mathrm{~B}$ & 257.51 & $90.10 \mathrm{~B}$ & 263.81 & $91.82 \mathrm{~B}$ \\
\hline 241.01 & $93.80 \mathrm{~B}$ & 246.71 & $93.02 \mathrm{~B}$ & 252.11 & $91.89 \mathrm{~B}$ & 257.81 & $91.91 \mathrm{~B}$ & 264.11 & $92.83 \mathrm{~B}$ \\
\hline 241.31 & $93.00 \mathrm{~B}$ & 247.01 & 90.71 B & 252.41 & 89.87 B & 258.77 & $92.95 \mathrm{~B}$ & 264.41 & $91.61 \mathrm{~B}$ \\
\hline 241.61 & $92.18 \mathrm{~B}$ & 247.31 & $93.88 \mathrm{~B}$ & 252.71 & $93.88 \mathrm{~B}$ & 259.01 & $93.06 \mathrm{~B}$ & 264.71 & $92.25 \mathrm{~B}$ \\
\hline 241.91 & $93.12 \mathrm{~B}$ & 247.61 & $93.77 \mathrm{~B}$ & 253.01 & $95.33 \mathrm{~B}$ & 259.31 & $92.46 \mathrm{~B}$ & 265.01 & $91.62 \mathrm{~B}$ \\
\hline 242.21 & $90.66 \mathrm{~B}$ & 247.91 & $91.39 \mathrm{~B}$ & 253.31 & $92.24 \mathrm{~B}$ & 259.61 & $94.43 \mathrm{~B}$ & 265.31 & $89.37 \mathrm{~B}$ \\
\hline 242.51 & $91.57 \mathrm{~B}$ & 248.21 & $88.83 \mathrm{~B}$ & 253.61 & $92.67 \mathrm{~B}$ & 259.91 & $92.77 \mathrm{~B}$ & 265.61 & $93.12 \mathrm{~B}$ \\
\hline 242.81 & $91.80 \mathrm{~B}$ & 248.51 & $93.15 \mathrm{~B}$ & 253.91 & $90.85 \mathrm{~B}$ & 260.23 & $94.81 \mathrm{~B}$ & 265.91 & $89.47 \mathrm{~B}$ \\
\hline 243.11 & $92.30 \mathrm{~B}$ & 248.81 & $90.29 \mathrm{~B}$ & 254.21 & $92.51 \mathrm{~B}$ & 260.51 & $93.53 \mathrm{~B}$ & 266.21 & $92.99 \mathrm{~B}$ \\
\hline 243.41 & $91.94 \mathrm{~B}$ & 249.07 & $92.85 \mathrm{~B}$ & 254.51 & 88.08 B & 260.81 & 96.55 B & 266.51 & $92.38 \mathrm{~B}$ \\
\hline 243.71 & $91.59 \mathrm{~B}$ & 249.11 & $91.49 \mathrm{~B}$ & 254.81 & $90.64 \mathrm{~B}$ & 261.11 & $92.26 \mathrm{~B}$ & 266.81 & $93.83 \mathrm{~B}$ \\
\hline 244.01 & $92.21 \mathrm{~B}$ & 249.41 & $90.77 \mathrm{~B}$ & 255.11 & $92.41 \mathrm{~B}$ & 261.41 & $90.23 \mathrm{~B}$ & 267.11 & $93.60 \mathrm{~B}$ \\
\hline 244.31 & $91.90 \mathrm{~B}$ & 249.71 & $93.70 \mathrm{~B}$ & 255.41 & $91.49 \mathrm{~B}$ & 261.71 & $92.73 \mathrm{~B}$ & 267.41 & 86.27 B \\
\hline 244.61 & $92.47 \mathrm{~B}$ & 250.01 & $94.05 \mathrm{~B}$ & 255.71 & $90.38 \mathrm{~B}$ & 262.01 & $92.98 \mathrm{~B}$ & 267.71 & $90.83 \mathrm{~B}$ \\
\hline 244.91 & $93.25 \mathrm{~B}$ & 250.31 & $94.23 \mathrm{~B}$ & 256.01 & $92.23 \mathrm{~B}$ & 262.31 & $93.89 \mathrm{~B}$ & 268.01 & $90.30 \mathrm{~B}$ \\
\hline 245.21 & $89.80 \mathrm{~B}$ & 250.61 & $86.29 \mathrm{~B}$ & 256.31 & $94.76 \mathrm{~B}$ & 262.61 & $94.82 \mathrm{~B}$ & & \\
\hline 245.51 & $87.05 \mathrm{~B}$ & 250.91 & 86.09 B & 256.61 & $93.48 \mathrm{~B}$ & 262.91 & $94.13 \mathrm{~B}$ & & \\
\hline 245.81 & $90.66 \mathrm{~B}$ & 251.21 & 89.83 B & 256.91 & $92.46 \mathrm{~B}$ & 263.21 & $95.03 \mathrm{~B}$ & & \\
\hline
\end{tabular}

Note: A "B" indicates values from Hole 609B, others are from Hole 609.

APPENDIX B

DSDP Site 607: Calcium Carbonate Values

\begin{tabular}{|c|c|c|c|c|c|c|c|c|c|}
\hline $\begin{array}{l}\text { Sub-bottom } \\
\text { depth } \\
\text { (m) }\end{array}$ & $\begin{array}{c}\mathrm{CaCO}_{3} \\
(\%)\end{array}$ & $\begin{array}{l}\text { Sub-bottom } \\
\text { depth } \\
\text { (m) }\end{array}$ & $\begin{array}{c}\mathrm{CaCO}_{3} \\
(\%)\end{array}$ & $\begin{array}{l}\text { Sub-bottom } \\
\text { depth } \\
\text { (m) }\end{array}$ & $\begin{array}{c}\mathrm{CaCO}_{3} \\
(\%)\end{array}$ & $\begin{array}{l}\text { Sub-bottom } \\
\text { depth } \\
\text { (m) }\end{array}$ & $\begin{array}{c}\mathrm{CaCO}_{3} \\
(\%)\end{array}$ & $\begin{array}{l}\text { Sub-bottom } \\
\text { depth } \\
\text { (m) }\end{array}$ & $\begin{array}{c}\mathrm{CaCO}_{3} \\
(\%)\end{array}$ \\
\hline 0.01 & 84.88 & 6.91 & 76.39 & 15.16 & 94.48 & 22.21 & 90.21 & 30.03 & 61.32 \\
\hline 0.16 & 82.25 & 7.06 & 69.20 & 15.31 & 92.25 & 22.36 & 91.04 & 30.23 & 63.66 \\
\hline 0.31 & 59.76 & 7.21 & 63.13 & 15.46 & 88.46 & 22.51 & 90.43 & 30.33 & 64.16 \\
\hline 0.53 & 53.05 & 7.36 & 76.52 & 15.61 & 91.00 & 22.73 & 85.00 & 30.48 & 70.33 \\
\hline 0.61 & 49.87 & 7.51 & 66.82 & 15.76 & 91.36 & 22.81 & 80.30 & 30.63 & 77.33 \\
\hline 0.78 & 50.21 & 7.51 & 74.18 & 15.91 & 96.37 & 22.98 & 80.11 & 30.76 & 75.05 \\
\hline 0.91 & 57.27 & 7.81 & 74.20 & 16.13 & 93.67 & 23.11 & 87.60 & 30.89 & 73.72 \\
\hline 1.06 & 53.70 & 7.88 & 80.84 & 16.21 & 93.65 & 23.26 & 82.81 & 31.06 & 85.63 \\
\hline 1.21 & 63.64 & 8.11 & 78.52 & 16.38 & 85.02 & 23.41 & 79.25 & 31.23 & 86.48 \\
\hline 1.36 & 62.02 & 8.13 & 81.57 & 16.51 & 93.54 & 23.56 & 84.60 & 31.36 & 84.83 \\
\hline 1.51 & 59.25 & 8.41 & 83.58 & 16.66 & 93.50 & 23.71 & 82.11 & 31.49 & 86.09 \\
\hline 1.66 & 67.51 & 8.56 & 69.83 & 16.81 & 90.26 & 23.86 & 82.80 & 31.73 & 80.66 \\
\hline 1.81 & 67.25 & 8.71 & 70.40 & 16.96 & 80.43 & 24.01 & 77.29 & 31.80 & 80.65 \\
\hline 2.03 & 59.69 & 8.86 & 81.51 & 17.11 & 52.67 & 24.23 & 68.31 & 31.98 & 63.57 \\
\hline 2.11 & 71.12 & 9.01 & 81.62 & 17.17 & 55.78 & 24.31 & 74.71 & 32.09 & 38.55 \\
\hline 2.28 & 75.44 & 9.16 & 73.16 & 17.26 & 65.82 & 24.48 & 78.78 & 32.26 & 52.03 \\
\hline 2.41 & 72.94 & 10.15 & 68.61 & 17.41 & 55.61 & 24.61 & 83.43 & 32.40 & 55.38 \\
\hline 2.56 & 68.52 & 10.38 & 64.69 & 17.63 & 58.35 & 25.52 & 78.73 & 32.56 & 66.20 \\
\hline 2.71 & 56.30 & 10.66 & 90.81 & 17.71 & 67.51 & 25.73 & 76.36 & 32.74 & 72.94 \\
\hline 2.86 & 60.40 & 10.96 & 91.19 & 17.88 & 74.63 & 25.82 & 78.97 & 32.86 & 77.47 \\
\hline 3.01 & 68.50 & 11.11 & 90.10 & 18.01 & 76.82 & 25.98 & 84.36 & 32.99 & 77.69 \\
\hline 3.16 & 74.82 & 11.26 & 79.45 & 18.16 & 77.43 & 26.13 & 84.98 & 33.23 & 75.64 \\
\hline 3.31 & 80.32 & 11,41 & 69.70 & 18.31 & 71.56 & 26.26 & 81.90 & 33.29 & 77.79 \\
\hline 3.53 & 84.57 & 11.63 & 86.20 & 18.46 & 67.18 & 26.42 & 80.54 & 33.48 & 63.57 \\
\hline 3.61 & 83.56 & 11.71 & 86.33 & 18.61 & 75.94 & 26.56 & 79.32 & 33.63 & 54.65 \\
\hline 3.78 & 82.78 & 11.88 & 86.18 & 18.76 & 75.05 & 26.72 & 78.92 & 33.76 & 60.50 \\
\hline 3.91 & 84.76 & 12.01 & 91.26 & 18.84 & 80.99 & 26.86 & 77.67 & 33.90 & 61.67 \\
\hline 4.06 & 87.05 & 12.16 & 89.41 & 19.21 & 92.82 & 27.02 & 73.95 & 34.06 & 64.84 \\
\hline 4.21 & 87.01 & 12.31 & 88.18 & 19.36 & 92.68 & 27.23 & 72.69 & 34.26 & 59.19 \\
\hline 4.36 & 86.49 & 12.46 & 91.19 & 19.51 & 84.82 & 27.32 & 69.58 & 34.36 & 71.01 \\
\hline 4.51 & 81.32 & 12.61 & 91.06 & 19.73 & 45.71 & 27.48 & 63.39 & 34.44 & 65.73 \\
\hline 4.66 & 82.61 & 12.76 & 88.28 & 19.81 & 64.32 & 27.63 & 55.06 & 34.83 & 77.81 \\
\hline 4.81 & 83.45 & 12.91 & 54.56 & 19.98 & 50.87 & 27.76 & 52.67 & 34.96 & 75.91 \\
\hline 5.03 & 74.99 & 13.13 & 63.06 & 20.11 & 64.63 & 27.92 & 46.52 & 35.09 & 79.11 \\
\hline 5.11 & 86.91 & 13.21 & 66.72 & 20.26 & 61.96 & 28.06 & 58.54 & 35.33 & 84.25 \\
\hline 5.20 & 87.29 & 13.38 & 70.59 & 20.41 & 71.72 & 28.23 & 64.96 & 35.41 & 83.74 \\
\hline 5.28 & 79.12 & 13.51 & 77.06 & 20.56 & 80.73 & 28.36 & 56.00 & 35.42 & 83.71 \\
\hline 5.41 & 61.81 & 13.66 & 79.08 & 20.71 & 78.49 & 28.52 & 61.49 & 35.58 & 90.13 \\
\hline 5.56 & 69.85 & 13.81 & 68.27 & 20.86 & 72.09 & 28.73 & 56.34 & 35.69 & 89.19 \\
\hline 5.71 & 69.67 & 13.96 & 59.15 & 21.01 & 62.50 & 28.83 & 57.45 & 35.86 & 86.81 \\
\hline 5.86 & 73.37 & 14.11 & 78.47 & 21.23 & 77.71 & 28.98 & 65.04 & 35.97 & 86.31 \\
\hline 6.01 & 70.73 & 14.26 & 77.71 & 21.31 & 73.53 & 29.14 & 65.71 & 35.99 & 86.03 \\
\hline 6.16 & 68.84 & 14.41 & 80.78 & 21.48 & 82.22 & 29.26 & 64.33 & 36.18 & 88.24 \\
\hline 6.31 & 56.21 & 14.63 & 86.14 & 21.61 & 80.05 & 29.42 & 70.20 & 36.34 & 89.73 \\
\hline 6.53 & 64.47 & 14.71 & 85.66 & 21.76 & 78.96 & 29.56 & 59.15 & 36.46 & 82.40 \\
\hline 6.61 & 63.52 & 14.88 & 91.86 & 21.91 & 86.54 & 29.73 & 69.07 & 36.61 & 86.49 \\
\hline 6.78 & 77.39 & 15.01 & 91.07 & 22.06 & 90.31 & 29.86 & 63.11 & 36.83 & 77.41 \\
\hline
\end{tabular}


W. F. RUDDIMAN, A. MCINTYRE, AND M. RAYMO

Appendix B (continued).

\begin{tabular}{|c|c|c|c|c|c|c|c|c|c|}
\hline $\begin{array}{l}\text { Sub-bottom } \\
\text { depth } \\
\text { (m) }\end{array}$ & $\begin{array}{c}\mathrm{CaCO}_{3} \\
(\%)\end{array}$ & $\begin{array}{l}\text { Sub-bottom } \\
\text { depth } \\
\text { (m) }\end{array}$ & $\begin{array}{c}\mathrm{CaCO}_{3} \\
(\%)\end{array}$ & $\begin{array}{l}\text { Sub-bottom } \\
\text { depth } \\
\text { (m) }\end{array}$ & $\begin{array}{c}\mathrm{CaCO}_{3} \\
(\%)\end{array}$ & $\begin{array}{l}\text { Sub-bottom } \\
\text { depth } \\
\text { (m) }\end{array}$ & $\begin{array}{c}\mathrm{CaCO}_{3} \\
(\%)\end{array}$ & $\begin{array}{l}\text { Sub-bottom } \\
\text { depth } \\
\text { (m) }\end{array}$ & $\begin{array}{c}\mathrm{CaCO}_{3} \\
(\%)\end{array}$ \\
\hline 36.88 & 66.74 & 47.70 & 73.11 & 59.28 & 86.04 & 70.96 & 87.01 & 83.33 & 86.13 \\
\hline 37.08 & 57.31 & 47.93 & 77.17 & 59.41 & 87.71 & 71.11 & 83.71 & 83.41 & 82.79 \\
\hline 37.19 & 60.74 & 47.99 & 85.13 & 59.56 & 95.02 & 71.26 & 73.96 & 83.58 & 88.84 \\
\hline 37.36 & 58.83 & 48.03 & 75.72 & 59.71 & 92.53 & 71.39 & 63.14 & 83.71 & 83.86 \\
\hline 37.49 & 60.82 & 48.18 & 83.70 & 59.86 & 92.66 & 71.63 & 70.38 & 83.86 & 90.65 \\
\hline 37.66 & 51.79 & 48.31 & 90.37 & 60.01 & 90.81 & 71.71 & 75.46 & 84.03 & 89.45 \\
\hline 37.83 & 39.36 & 48.46 & 91.36 & 60.16 & 82.62 & 71.88 & 86.00 & 84.16 & 74.40 \\
\hline 37.96 & 49.07 & 48.59 & 91.93 & 60.30 & 59.80 & 71.99 & 81.30 & 84.17 & 75.40 \\
\hline 38.09 & 59.07 & 48.76 & 89.86 & 60.37 & 64.14 & 72.16 & 82.04 & 84.31 & 88.42 \\
\hline 38.33 & 76.59 & 48.94 & 91.89 & 60.53 & 68.30 & 72.31 & 85.52 & 84.46 & 92.06 \\
\hline 38.38 & 79.06 & 49.06 & 91.38 & 60.61 & 72.40 & 72.46 & 86.38 & 84.61 & 93.03 \\
\hline 38.41 & 79.06 & 49.19 & 89.24 & 60.78 & 79.33 & 72.61 & 81.22 & 84.83 & 92.04 \\
\hline 38.58 & 84.26 & 49.20 & 89.82 & 60.91 & 84.35 & 72.76 & 80.42 & 84.89 & 96.02 \\
\hline 38.74 & 77.63 & 49.43 & 88.99 & 61.06 & 89.49 & 73.21 & 92.61 & 85.08 & 92.95 \\
\hline 38.86 & 83.80 & 49.50 & 91.10 & 61.21 & 89.50 & 73.36 & 93.77 & 85.19 & 94.18 \\
\hline 38.99 & 80.20 & 49.68 & 89.19 & 61.36 & 92.07 & 73.73 & 90.72 & 85.36 & 90.56 \\
\hline 39.16 & 64.60 & 49.81 & 88.65 & 61.51 & 90.98 & 73.81 & 89.98 & 85.49 & 91.27 \\
\hline 39.33 & 65.64 & 49.96 & 78.56 & 61.66 & 92.17 & 73.98 & 88.88 & 85.66 & 88.44 \\
\hline 39.46 & 62.66 & 50.09 & 83.38 & 61.81 & 86.48 & 74.26 & 71.13 & 85.83 & 90.09 \\
\hline 39.59 & 67.45 & 50.11 & 89.25 & 62.03 & 73.48 & 74.41 & 71.81 & 85.96 & 94.40 \\
\hline 39.83 & 66.02 & 50.25 & 70.14 & 62.11 & 74.49 & 74.56 & 76.29 & 86.11 & 91.93 \\
\hline 39.89 & 73.26 & 50.43 & 79.14 & 62.28 & 77.87 & 74.71 & 78.38 & 86.33 & 91.09 \\
\hline 39.90 & 76.36 & 50.56 & 81.28 & 62.41 & 76.38 & 74.86 & 85.20 & 86.43 & 93.33 \\
\hline 40.08 & 86.91 & 50.70 & 75.99 & 62.56 & 82.53 & 75.01 & 88.52 & 86.58 & 92.08 \\
\hline 40.19 & 87.43 & 50.93 & 78.85 & 62.71 & 87.39 & 75.23 & 88.37 & 86.71 & 87.72 \\
\hline 40.36 & 91.49 & 50.99 & 77.85 & 62.86 & 91.27 & 75.31 & 87.66 & 86.86 & 78.18 \\
\hline 40.49 & 93.27 & 51.18 & 74.58 & 63.01 & 91.06 & 75.48 & 87.04 & 87.01 & 75.78 \\
\hline 40.52 & 93.24 & 51.31 & 86.29 & 63.16 & 92.16 & 75.61 & 89.10 & 87.16 & 76.15 \\
\hline 40.61 & 92.22 & 51.46 & 91.33 & 63.24 & 89.92 & 75.76 & 91.04 & 87.33 & 73.13 \\
\hline 40.83 & 91.07 & 51.60 & 90.35 & 63.65 & 80.39 & 75.91 & 88.76 & 87.46 & 74.88 \\
\hline 40.96 & 90.62 & 51.76 & 91.69 & 63.76 & 76.31 & 76.06 & 87.90 & 87.61 & 69.38 \\
\hline 41.09 & 85.32 & 51.93 & 86.02 & 63.91 & 73.96 & 76.21 & 78.98 & 87.83 & 80.36 \\
\hline 41.17 & 79.71 & 52.06 & 73.11 & 64.13 & 78.24 & 76.36 & 79.90 & 87.89 & 83.46 \\
\hline 41.33 & 68.78 & 52.22 & 65.07 & 64.21 & 82.11 & 76.51 & 85.24 & 88.08 & 89.71 \\
\hline 41.41 & 81.14 & 52.43 & 63.63 & 64.38 & 86.75 & 76.73 & 86.79 & 88.19 & 90.62 \\
\hline 41.58 & 85.27 & 52.49 & 63.45 & 64.49 & 90.85 & 76.81 & 88.87 & 88.28 & 84.65 \\
\hline 41.71 & 86.27 & 52.68 & 74.69 & 64.66 & 81.59 & 76.98 & 86.49 & 88.36 & 76.35 \\
\hline 41.86 & 86.09 & 52.79 & 69.83 & 64.81 & 86.45 & 77.11 & 89.13 & 88.46 & 55.56 \\
\hline 41.99 & 83.09 & 52.96 & 85.72 & 64.96 & 92.07 & 77.26 & 90.01 & 88.53 & 83.81 \\
\hline 42.16 & 76.20 & 53.09 & 87.92 & 65.13 & 92.88 & 77.43 & 91.99 & 88.66 & 87.10 \\
\hline 42.24 & 85.51 & 53.26 & 90.95 & 65.26 & 93.71 & 77.56 & 89.40 & 88.83 & 90.65 \\
\hline 42.33 & 84.84 & 53.43 & 82.32 & 65.39 & 91.94 & 77.73 & 92.17 & 88.96 & 90.05 \\
\hline 42.46 & 87.40 & 53.59 & 90.13 & 65.63 & 87.12 & 77.86 & 85.21 & 89.15 & 89.64 \\
\hline 42.59 & 87.95 & 54.05 & 80.34 & 65.71 & 85.62 & 78.01 & 90.29 & 89.33 & 85.68 \\
\hline 42.83 & 87.86 & 54.16 & 86.96 & 65.88 & 86.23 & 78.23 & 89.97 & 89.41 & 83.15 \\
\hline 42.88 & 89.08 & 54.29 & 87.18 & 66.01 & 88.02 & 78.31 & 92.05 & 89.58 & 84.56 \\
\hline 43.08 & 77.90 & 54.53 & 88.14 & 66.16 & 88.66 & 78.48 & 91.37 & 89.71 & 88.71 \\
\hline 43.18 & 83.87 & 54.61 & 88.97 & 66.29 & 88.16 & 78.61 & 90.61 & 89.86 & 90.48 \\
\hline 43.20 & 82.08 & 54.78 & 88.03 & 66.46 & 88.79 & 78.76 & 94.90 & 90.01 & 91.34 \\
\hline 43.36 & 84.87 & 54.91 & 85.33 & 66.61 & 84.22 & 78.91 & 94.39 & 90.16 & 89.94 \\
\hline 43.50 & 82.85 & 55.06 & 85.12 & 66.76 & 77.01 & 79.06 & 93.50 & 90.31 & 89.98 \\
\hline 43.66 & 87.88 & 55.21 & 80.51 & 66.91 & 88.15 & 79.21 & 90.77 & 90.46 & 91.75 \\
\hline 43.83 & 91.68 & 55.36 & 66.03 & 67.13 & 88.66 & 79.36 & 90.80 & 90.61 & 84.65 \\
\hline 43.96 & 92.00 & 55.36 & 67.17 & 67.23 & 90.38 & 79.51 & 81.21 & 90.83 & 77.03 \\
\hline 44.01 & 89.90 & 55.53 & 68.27 & 67.38 & 88.63 & 79.73 & 81.14 & 90.89 & 77.02 \\
\hline 44.56 & 72.93 & 55.66 & 76.73 & 67.49 & 93.35 & 79.81 & 80.22 & 91.08 & 76.04 \\
\hline 44.72 & 74.68 & 55.81 & 76.37 & 67.66 & 90.30 & 79.98 & 82.09 & 91.19 & 71.10 \\
\hline 44.93 & 85.24 & 56.03 & 85.21 & 67.79 & 88.05 & 80.11 & 83.25 & 91.36 & 69.56 \\
\hline 45.03 & 90.78 & 56.28 & 91.14 & 67.96 & 89.02 & 80.26 & 82.57 & 91.49 & 78.13 \\
\hline 45.18 & 92.40 & 56.41 & 89.64 & 68.11 & 89.53 & 80.41 & 84.74 & 91.66 & 81.78 \\
\hline 45.33 & 91.39 & 56.56 & 90.46 & 68.26 & 86.17 & 80.56 & 85.65 & 91.83 & 91.43 \\
\hline 45.46 & 90.80 & 56.71 & 88.81 & 68.41 & 87.67 & 80.71 & 87.07 & 91.96 & 92.79 \\
\hline 45.58 & 92.30 & 56.86 & 89.50 & 68.63 & 90.18 & 80.86 & 89.68 & 92.07 & 92.02 \\
\hline 45.76 & 87.55 & 57.01 & 89.27 & 68.71 & 91.56 & 80.99 & 90.07 & 92.45 & 89.82 \\
\hline 45.94 & 89.17 & 57.16 & 86.15 & 68.88 & 87.66 & 81.23 & 88.37 & 92.56 & 91.02 \\
\hline 46.06 & 88.41 & 57.29 & 82.07 & 69.01 & 85.71 & 81.31 & 89.68 & 92.71 & 87.60 \\
\hline 46.20 & 85.39 & 57.53 & 75.80 & 69.16 & 82.59 & 81.48 & 89.63 & 92.93 & 87.03 \\
\hline 46.43 & 68.79 & 57.63 & 79.65 & 69.31 & 79.76 & 81.59 & 89.37 & 93.01 & 89.13 \\
\hline 46.45 & 72.66 & 57.78 & 74.70 & 69.46 & 81.73 & 81.76 & 86.30 & 93.18 & 90.95 \\
\hline 46.49 & 77.86 & 57.91 & 85.25 & 69.61 & 80.84 & 81.91 & 84.38 & 93.31 & 90.79 \\
\hline 46.68 & 86.80 & 58.06 & 80.57 & 69.76 & 72.58 & 82.06 & 82.69 & 93.46 & 91.56 \\
\hline 46.77 & 86.68 & 58.21 & 92.59 & 69.91 & 75.99 & 82.21 & 81.01 & 93.61 & 87.02 \\
\hline 46.79 & 81.83 & 58.36 & 91.53 & 70.13 & 85.53 & 82.36 & 87.16 & 93.76 & 82.49 \\
\hline 46.96 & 84.11 & 58.41 & 86.27 & 70.21 & 87.60 & 82.51 & 83.35 & 93.91 & 74.94 \\
\hline 47.10 & 79.27 & 58.66 & 88.27 & 70.38 & 90.32 & 82.87 & 84.02 & 94.06 & 75.87 \\
\hline 47.26 & 72.46 & 58.79 & 85.60 & 70.51 & 88.58 & 82.96 & 93.98 & 94.21 & 75.77 \\
\hline 47.44 & 64.91 & 59.03 & 80.52 & 70.66 & 90.22 & 82.96 & 90.37 & 94.43 & 82.92 \\
\hline 47.56 & 73.31 & 59.09 & 82.10 & 70.81 & 89.27 & 83.11 & 87.80 & 94.51 & 86.65 \\
\hline
\end{tabular}


Appendix B (continued).

\begin{tabular}{|c|c|c|c|c|c|c|c|c|c|}
\hline $\begin{array}{l}\text { Sub-bottom } \\
\text { depth } \\
\text { (m) }\end{array}$ & $\begin{array}{c}\mathrm{CaCO}_{3} \\
(\%)\end{array}$ & $\begin{array}{l}\text { Sub-bottom } \\
\text { depth } \\
\text { (m) }\end{array}$ & $\underset{(\%)}{\mathrm{CaCO}_{3}}$ & $\begin{array}{l}\text { Sub-bottom } \\
\text { depth } \\
\text { (m) }\end{array}$ & $\begin{array}{c}\mathrm{CaCO}_{3} \\
(\%)\end{array}$ & $\begin{array}{l}\text { Sub-bottom } \\
\text { depth } \\
\text { (m) }\end{array}$ & $\begin{array}{c}\mathrm{CaCO}_{3} \\
(\%)\end{array}$ & $\begin{array}{l}\text { Sub-bottom } \\
\text { depth } \\
\text { (m) }\end{array}$ & $\begin{array}{c}\mathrm{CaCO}_{3} \\
(\%)\end{array}$ \\
\hline 94.68 & 91.36 & 106.36 & 74.89 & 118.21 & 87.08 & 130.08 & 92.54 & 142.51 & 94.02 \\
\hline 94.81 & 93.33 & 106.51 & 67.90 & 118.38 & 88.08 & 130.23 & 94.97 & 142.68 & 96.18 \\
\hline 94.96 & 92.30 & 106.66 & 70.43 & 118.51 & 94.01 & 130.38 & 94.26 & 142.81 & 94.04 \\
\hline 95.09 & 89.85 & 106.79 & 67.34 & 118.66 & 93.06 & 130.81 & 87.33 & 142.96 & 98.72 \\
\hline 95.26 & 80.44 & 107.03 & 77.93 & 118.81 & 92.33 & 130.96 & 87,70 & 143.11 & 94.37 \\
\hline 95.26 & 83.46 & 107.11 & 77.69 & 118.96 & 93.13 & 131.11 & 88.90 & 143.26 & 91.83 \\
\hline 95.41 & 89.42 & 107.28 & 85.51 & 119.11 & 90.84 & 131.33 & 90.70 & 143.41 & 92.77 \\
\hline 95.56 & 91.03 & 107.39 & 89.70 & 119.26 & 93.13 & 131.41 & 89.93 & 143.56 & 94.49 \\
\hline 95.71 & 85.95 & 107.56 & 93.38 & 119.39 & 92.26 & 131.71 & 94.56 & 143.71 & 95.46 \\
\hline 95.93 & 74.08 & 107.71 & 92.89 & 119.63 & 87.44 & 131.86 & 94.89 & 143.93 & 96.58 \\
\hline 96.03 & 79.03 & 107.86 & 91.85 & 119.71 & 92.92 & 132.01 & 91.44 & 144.03 & 94.62 \\
\hline 96.18 & 74.08 & 108.01 & 91.79 & 119.88 & 88.75 & 132.16 & 92.16 & 144.03 & 96.36 \\
\hline 96.31 & 74.99 & 108.16 & 92.87 & 120.01 & 89.93 & 132.31 & 91.34 & 144.18 & 95.94 \\
\hline 96.46 & 80.86 & 108.32 & 93.04 & 120.16 & 92.95 & 132.46 & 93.33 & 144.33 & 87.35 \\
\hline 96.61 & 87.31 & 108.53 & 88.87 & 120.31 & 92.82 & 132.61 & 89.88 & 144.33 & 88.83 \\
\hline 96.76 & 89.05 & 108.61 & 90.82 & 120.46 & 93.21 & 132.83 & 91.46 & 144.46 & 93.41 \\
\hline 96.91 & 89.42 & 108.78 & 89.72 & 120.61 & 90.54 & 132.89 & 93.04 & 144.61 & 93.90 \\
\hline 97.06 & 91.67 & 108.91 & 91.14 & 120.76 & 92.50 & 133.08 & 92.83 & 144.76 & 96.37 \\
\hline 97.21 & 87.53 & 109.06 & 91.31 & 120.81 & 90.87 & 133.19 & 95.60 & 144.91 & 94.50 \\
\hline 97.43 & 87.87 & 109.21 & 90.33 & 121.25 & 93.38 & 133.36 & 95.44 & 145.06 & 92.92 \\
\hline 97.51 & 88.46 & 109.36 & 91.29 & 121.36 & 88.58 & 133.49 & 96.11 & 145.21 & 92.28 \\
\hline 97.68 & 89.10 & 109.51 & 90.86 & 121.51 & 92.27 & 133.66 & 98.84 & 145.31 & 95.68 \\
\hline 97.81 & 85.15 & 109.66 & 87.29 & 121.73 & 91.14 & 133.81 & 92.76 & 145.51 & 94.64 \\
\hline 97.96 & 85.69 & 109.81 & 87.78 & 121.81 & 92.70 & 133.96 & 94.04 & 145.59 & 93.19 \\
\hline 98.11 & 76.72 & 110.03 & 77.98 & 121.98 & 91.72 & 134.11 & 92.58 & 145.81 & 92.75 \\
\hline 98.11 & 76.55 & 110.11 & 67.68 & 122.11 & 97.13 & 134.33 & 92.96 & 145.87 & 90.42 \\
\hline 98.26 & 84.65 & 110.28 & 74.10 & 122.26 & 92.49 & 134.44 & 93.15 & 146.13 & 88.80 \\
\hline 98.43 & 85.44 & 110.41 & 75.02 & 122.41 & 88.66 & 134.58 & 92.86 & 146.13 & 91.50 \\
\hline 98.58 & 89.16 & 110.56 & 84.04 & 122.56 & 85.44 & 134.71 & 91.97 & 146.26 & 93.70 \\
\hline 98.71 & 91.70 & 110.71 & 89.02 & 122.71 & 91.40 & 134.86 & 93.93 & 148.66 & 93.12 \\
\hline 98.93 & 91.53 & 110.86 & 91.39 & 122.86 & 87.69 & 135.01 & 93.63 & 150.16 & 92.27 \\
\hline 99.01 & 89.84 & 111.07 & 94.88 & 122.99 & 89.26 & 135.16 & 93.39 & 151.66 & 93.42 \\
\hline 99.18 & 86.20 & 111.67 & 94.11 & 123.23 & 90.74 & 135.31 & 88.46 & 152.01 & 94.15 \\
\hline 99.31 & 83.68 & 111.76 & 91.97 & 123.29 & 91.24 & 135.46 & 92.60 & 153.16 & 93.79 \\
\hline 99.46 & 87.33 & 111.91 & 86.40 & 123.48 & 91.58 & 135.61 & 91.31 & 153.39 & 91.46 \\
\hline 99.61 & 82.58 & 112.13 & 91.87 & 123.61 & 91.05 & 135.83 & 91.61 & 154.76 & 92.55 \\
\hline 99.76 & 77.26 & 112.21 & 86.45 & 123.76 & 92.64 & 135.91 & 94.89 & 155.11 & 92.72 \\
\hline 99.91 & 74.61 & 112.38 & 92.17 & 123.91 & 92.04 & 136.08 & 90.87 & 159.61 & 92.27 \\
\hline 100.06 & 86.38 & 112.51 & 92.37 & 124.06 & 91.59 & 136.21 & 91.54 & 159.91 & 91.39 \\
\hline 100.21 & 89.72 & 112.66 & 92.32 & 124.21 & 89.63 & 136.36 & 91.13 & 160.21 & 92.32 \\
\hline 100.43 & 90.19 & 112.79 & 91.09 & 124.36 & 92.22 & 136.53 & 87.61 & 160.51 & 90.63 \\
\hline 100.51 & 88.92 & 112.96 & 86.68 & 124.49 & 90.09 & 136.66 & 90.75 & 160.81 & 92.75 \\
\hline 100.69 & 89.88 & 113.11 & 86.20 & 124.73 & 87.09 & 136.81 & 90.09 & 161.11 & 93.60 \\
\hline 100.81 & 88.04 & 113.26 & 84.02 & 124.81 & 87.39 & 136.96 & 90.09 & 161.41 & 91.76 \\
\hline 100.96 & 90.78 & 113.41 & 88.76 & 124.90 & 87.21 & 137.11 & 87.58 & 161.71 & 89.58 \\
\hline 101.11 & 85.72 & 113.63 & 87.51 & 125.11 & 89.69 & 137.33 & 88.78 & 162.01 & 93.43 \\
\hline 101.26 & 91.27 & 113.71 & 87.23 & 125.26 & 89.83 & 137.41 & 88.02 & 162.31 & 93.94 \\
\hline 101.45 & 86.08 & 113.88 & 88.78 & 125.41 & 88.22 & 137.58 & 91.16 & 162.61 & 93.15 \\
\hline 101.48 & 90.02 & 114.01 & 84.54 & 125.56 & 89.94 & 137.71 & 92.44 & 162.91 & 92.73 \\
\hline 102.05 & 91.56 & 114.16 & 85.16 & 125.73 & 88.46 & 137.86 & 94.26 & 163.16 & 92.67 \\
\hline 102.16 & 87.98 & 114.31 & 83.65 & 125.86 & 90.39 & 138.01 & 92.56 & 163.16 & 92.69 \\
\hline 102.31 & 80.29 & 114.46 & 81.15 & 126.01 & 88.28 & 138.16 & 94.95 & 163.21 & 93.70 \\
\hline 102.53 & 79.37 & 114.61 & 79.41 & 126.23 & 87.10 & 138.31 & 94.60 & 163.51 & 91.41 \\
\hline 102.61 & 78.20 & 114.76 & 81.46 & 126.31 & 86.03 & 138.46 & 94.58 & 163.81 & 94.36 \\
\hline 102.78 & 65.50 & 114.91 & 88.33 & 126.48 & 85.97 & 138.83 & 93.34 & 164.11 & 96.55 \\
\hline 102.91 & 76.61 & 115.02 & 82.45 & 126.59 & 85.37 & 138.91 & 93.24 & 164.41 & 92.93 \\
\hline 103.06 & 69.91 & 115.13 & 80.46 & 126.76 & 85.55 & 139.08 & 94.46 & 164.71 & 92.71 \\
\hline 103.21 & 75.97 & 115.14 & 83.51 & 126.91 & 87.51 & 139.21 & 93.60 & 165.01 & 92.86 \\
\hline 103.36 & 75.87 & 115.21 & 92.70 & 127.06 & 89.62 & 139.36 & 94.72 & 165.31 & 89.86 \\
\hline 103.51 & 82.60 & 115.38 & 89.37 & 127.24 & 89.64 & 139.51 & 91.59 & 165.61 & 94.01 \\
\hline 103.66 & 75.87 & 115.51 & 89.57 & 127.36 & 89.97 & 139.66 & 92.83 & 165.91 & 91.04 \\
\hline 103.81 & 89.66 & 115.66 & 91.50 & 127.51 & 88.49 & 139.81 & 98.22 & 166.21 & 93.19 \\
\hline 104.03 & 90.70 & 115.78 & 90.86 & 127.73 & 87.99 & 139.96 & 94.88 & 166.51 & 93.56 \\
\hline 104.11 & 91.33 & 115.96 & 91.17 & 127.81 & 88.51 & 140.11 & 94.35 & 166.81 & 94.45 \\
\hline 104.28 & 90.76 & 116.11 & 91.73 & 127.89 & 88.39 & 140.41 & 94.91 & 167.11 & 92.87 \\
\hline 104.41 & 90.57 & 116.26 & 89.43 & 128.11 & 90.71 & 140.56 & 93.35 & 167.41 & 92.68 \\
\hline 104.56 & 87.42 & 116.41 & 80.58 & 128.26 & 90.50 & 140.71 & 92.10 & 167.71 & 93.89 \\
\hline 104.71 & 83.71 & 116.63 & 87.82 & 128.39 & 91.45 & 140.93 & 92.80 & 167.99 & 93.02 \\
\hline 104.86 & 62.59 & 116.69 & 90.48 & 128.56 & 92.24 & 141.01 & 93.11 & 182.36 & 92.53 \\
\hline 105.01 & 72.23 & 116.88 & 90.91 & 128.71 & 90.32 & 141.18 & 95.41 & 191.96 & 93.71 \\
\hline 105.16 & 79.14 & 117.01 & 92.19 & 128.86 & 93.22 & 141.31 & 93.16 & 201.56 & 92.88 \\
\hline 105.31 & 78.99 & 117.16 & 91.27 & 128.99 & 96.23 & 141.46 & 92.21 & 211.16 & 90.98 \\
\hline 105.53 & 88.23 & 117.31 & 90.73 & 129.23 & 92.04 & 141.61 & 93.14 & 220.76 & 94.24 \\
\hline 105.61 & 90.22 & 117.46 & 90.78 & 129.31 & 93.61 & 141.76 & 93.58 & 230.33 & 95.17 \\
\hline 105.78 & 90.63 & 117.61 & 86.30 & 129.48 & 90.03 & 141.91 & 95.75 & 239.93 & 95.18 \\
\hline 105.89 & 89.20 & 117.76 & 84.50 & 129.59 & 92.23 & 142.06 & 93.88 & & \\
\hline 106.06 & 88.31 & 117.91 & 87.05 & 129.78 & 92.65 & 142.17 & 98.03 & & \\
\hline 106.21 & 77.56 & 118.13 & 83.63 & 129.91 & 91.31 & 142.43 & 96.02 & & \\
\hline
\end{tabular}


APPENDIX C

DSDP Site 607: Oxygen Isotope and Faunal Data

\begin{tabular}{|c|c|c|c|c|c|c|c|}
\hline $\begin{array}{l}\text { Sub-bottom } \\
\text { depth } \\
\text { (m) }\end{array}$ & $\begin{array}{c}\delta^{18} \mathrm{O} \\
\left(\%_{0}\right)\end{array}$ & $\begin{array}{c}G . \\
\text { ruber } \\
(\%)\end{array}$ & $\begin{array}{c}G . \\
\text { bulloid. } \\
(\%)\end{array}$ & $\begin{array}{c}\text { N. } \\
\text { pachy. s. } \\
(\%)\end{array}$ & $\begin{array}{c}G . \\
\text { inflata } \\
(\%)\end{array}$ & $\begin{array}{c}\text { All } \\
\text { other } \\
(\%)\end{array}$ & $\begin{array}{l}\text { SSTw } \\
\left({ }^{\circ} \mathrm{C}\right)\end{array}$ \\
\hline 9.61 & $4.35 \mathrm{U}$ & 0.7 & 14.1 & 1.0 & 8.2 & 75.9 & 12.1 \\
\hline 9.76 & $3.84 \mathrm{U}$ & & & & & & \\
\hline 9.76 & $4.33 \mathrm{C}$ & & & & & & \\
\hline 9.91 & & 4.7 & 15.7 & 1.7 & 22.3 & 55.6 & 13.5 \\
\hline 10.15 & $3.94 \mathrm{C}$ & 4.0 & 15.0 & 7.2 & 7.6 & 66.2 & 11.6 \\
\hline 10.15 & $4.35 \mathrm{U}$ & & & & & & \\
\hline 10.21 & $3.21 \mathrm{U}$ & 8.8 & 10.7 & 1.7 & 28.3 & 50.6 & 16.4 \\
\hline 10.38 & $4.24 \mathrm{U}$ & 1.9 & 14.2 & 4.7 & 9.4 & 69.8 & 11.6 \\
\hline 10.38 & $2.74 \mathrm{C}$ & & & & & & \\
\hline 10.51 & $3.81 \mathrm{U}$ & 2.2 & 21.1 & 4.4 & 15.6 & 56.7 & 10.4 \\
\hline 10.66 & $3.25 \mathrm{C}$ & 2.7 & 9.7 & 0.3 & 18.2 & 69.0 & 13.8 \\
\hline 10.66 & $4.33 \mathrm{U}$ & & & & & & \\
\hline 10.66 & $3.65 \mathrm{C}$ & & & & & & \\
\hline 10.81 & $2.87 \mathrm{C}$ & 3.0 & 12.2 & 2.7 & 21.6 & 60.5 & 13.0 \\
\hline 10.96 & $2.25 \mathrm{C}$ & 4.0 & 13.2 & 1.8 & 23.5 & 57.4 & 13.6 \\
\hline 11.11 & $3.28 \mathrm{C}$ & 6.2 & 13.1 & 1.4 & 13.1 & 66.2 & 14.2 \\
\hline 11.26 & $2.88 \mathrm{C}$ & 2.2 & 15.5 & 8.5 & 9.6 & 64.2 & 10.6 \\
\hline 11.41 & & 4.4 & 14.6 & 10.2 & 13.5 & 57.3 & 11.2 \\
\hline 11.63 & $3.46 \mathrm{C}$ & 2.5 & 11.3 & 2.6 & 21.1 & 62.4 & 12.9 \\
\hline 11.63 & $3.21 \mathrm{C}$ & & & & & & \\
\hline 11.71 & $3.12 \mathrm{C}$ & 1.1 & 13.5 & 2.2 & 11.3 & 72.0 & 12.0 \\
\hline 11.88 & $2.99 \mathrm{C}$ & 4.1 & 17.4 & 2.2 & 16.5 & 59.8 & 12.6 \\
\hline 12.01 & $3.06 \mathrm{C}$ & 4.3 & 8.7 & 2.3 & 18.3 & 66.4 & 14.0 \\
\hline 12.16 & $2.52 \mathrm{C}$ & 2.8 & 16.0 & 2.8 & 16.5 & 61.9 & 12.1 \\
\hline 12.31 & $2.37 \mathrm{C}$ & 2.1 & 19.5 & 1.7 & 9.6 & 67.1 & 11.4 \\
\hline 12.38 & & 2.0 & 18.9 & 5.1 & 9.5 & 64.5 & 10.7 \\
\hline 12.46 & $2.78 \mathrm{C}$ & 2.2 & 8.0 & 2.5 & 21.4 & 65.9 & 13.4 \\
\hline 12.46 & $2.45 \mathrm{C}$ & & & & & & \\
\hline 12.61 & $2.13 \mathrm{C}$ & 10.5 & 8.4 & 1.7 & 19.6 & 59.8 & 16.4 \\
\hline 12.61 & $2.30 \mathrm{C}$ & & & & & & \\
\hline 12.76 & $2.58 \mathrm{C}$ & 10.0 & 10.4 & 5.2 & 20.4 & 54.1 & 15.2 \\
\hline 12.91 & $2.57 \mathrm{C}$ & 3.0 & 16.9 & 13.9 & 8.6 & 57.5 & 9.6 \\
\hline 13.13 & $3.17 \mathrm{C}$ & 1.4 & 30.9 & 20.7 & 7.9 & 39.0 & 5.4 \\
\hline 13.13 & $4.46 \mathrm{U}$ & & & & & & \\
\hline 13.13 & $2.87 \mathrm{C}$ & & & & & & \\
\hline 13.21 & $3.81 \mathrm{C}$ & 1.2 & 8.3 & 4.7 & 22.4 & 63.4 & 12.3 \\
\hline 13.21 & $4.98 \mathrm{U}$ & & & & & & \\
\hline 13.38 & $3.88 \mathrm{C}$ & 1.6 & 23.5 & 8.7 & 9.1 & 57.1 & 8.7 \\
\hline 13.51 & $5.02 \mathrm{U}$ & 1.2 & 10.8 & 4.8 & 15.2 & 68.0 & 11.8 \\
\hline 13.51 & $3.78 \mathrm{C}$ & & & & & & \\
\hline 13.66 & $2.82 \mathrm{C}$ & 1.1 & 13.9 & 10.1 & 8.2 & 66.7 & 10.2 \\
\hline 13.66 & $4.38 \mathrm{U}$ & & & & & & \\
\hline 13.81 & $3.80 \mathrm{C}$ & 0.7 & 32.2 & 7.3 & 5.0 & 54.8 & 7.0 \\
\hline 13.96 & $3.48 \mathrm{C}$ & 0.0 & 39.9 & 16.1 & 0.5 & 43.4 & 4.1 \\
\hline 14.11 & $4.55 \mathrm{U}$ & 0.4 & 15.4 & 9.4 & 5.6 & 69.2 & 9.9 \\
\hline 14.11 & $2.68 \mathrm{C}$ & & & & & & \\
\hline 14.26 & $3.06 \mathrm{C}$ & 1.3 & 19.9 & 5.0 & 9.3 & 64.5 & 10.2 \\
\hline 14.33 & $4.39 \mathrm{U}$ & 2.1 & 22.4 & 4.2 & 8.8 & 62.5 & 10.2 \\
\hline 14.41 & $2.87 \mathrm{C}$ & 2.4 & 14.0 & 5.9 & 11.9 & 65.7 & 11.5 \\
\hline 14.49 & $3.63 \mathrm{C}$ & 2.3 & 13.4 & 9.2 & 10.7 & 64.4 & 10.8 \\
\hline 14.63 & $3.45 \mathrm{U}$ & 3.1 & 13.5 & 4.1 & 12.6 & 66.7 & 12.3 \\
\hline 14.63 & $3.30 \mathrm{C}$ & & & & & & \\
\hline 14.71 & $3.45 \mathrm{C}$ & 3.9 & 10.2 & 3.3 & 14.0 & 68.7 & 13.3 \\
\hline 14.71 & $3.72 \mathrm{U}$ & & & & & & \\
\hline 14.88 & $3.16 \mathrm{C}$ & 2.2 & 10.3 & 9.6 & 16.8 & 61.1 & 11.2 \\
\hline 14.88 & $4.53 \mathrm{U}$ & & & & & & \\
\hline 14.88 & $3.59 \mathrm{C}$ & & & & & & \\
\hline 14.88 & $4.27 \mathrm{U}$ & & & & & & \\
\hline 15.01 & $3.13 \mathrm{C}$ & 5.7 & 7.9 & 3.9 & 22.1 & 60.4 & 14.4 \\
\hline 15.01 & $3.05 \mathrm{U}$ & & & & & & \\
\hline 15.16 & $3.96 \mathrm{U}$ & 2.8 & 9.9 & 1.2 & 18.5 & 67.6 & 13.6 \\
\hline 15.16 & $3.37 \mathrm{C}$ & & & & & & \\
\hline 15.16 & $3.82 \mathrm{U}$ & & & & & & \\
\hline 15.31 & $3.88 \mathrm{U}$ & 2.6 & 14.5 & 1.5 & 17.5 & 63.9 & 12.6 \\
\hline 15.46 & $1.80 \mathrm{C}$ & 2.4 & 11.6 & 10.4 & 19.0 & 56.7 & 10.8 \\
\hline 15.46 & $3.43 \mathrm{U}$ & & & & & & \\
\hline 15.61 & $2.24 \mathrm{C}$ & 5.1 & 6.6 & 1.1 & 39.6 & 47.6 & 16.9 \\
\hline 15.61 & $2.34 \mathrm{U}$ & & & & & & \\
\hline 15.76 & $3.22 \mathrm{U}$ & 5.6 & 12.6 & 2.7 & 32.9 & 46.2 & 15.2 \\
\hline 15.76 & $2.55 \mathrm{C}$ & & & & & & \\
\hline 15.83 & & 11.7 & 10.6 & 4.2 & 29.9 & 43.6 & 17.1 \\
\hline 15.91 & $2.55 \mathrm{C}$ & 12.5 & 7.7 & 0.3 & 27.9 & 51.5 & 18.3 \\
\hline 15.91 & $2.65 \mathrm{C}$ & & & & & & \\
\hline 16.13 & $2.40 \mathrm{C}$ & 22.2 & 8.9 & 0.6 & 24.2 & 44.1 & 20.2 \\
\hline 16.21 & $2.17 \mathrm{C}$ & 16.6 & 6.7 & 1.1 & 25.8 & 49.8 & 19.1 \\
\hline 16.21 & $2.07 \mathrm{C}$ & & & & & & \\
\hline 16.28 & & 14.6 & 8.9 & 1.4 & 21.0 & 54.1 & 17.8 \\
\hline 16.38 & $2.54 \mathrm{C}$ & 17.2 & 8.3 & 1.0 & 22.1 & 51.4 & 18.7 \\
\hline 16.38 & $2.23 \mathrm{C}$ & & & & & & \\
\hline 16.51 & $2.69 \mathrm{C}$ & 24.6 & 7.2 & 0.6 & 15.3 & 52.3 & 19.5 \\
\hline
\end{tabular}

Appendix C (continued).

\begin{tabular}{|c|c|c|c|c|c|c|c|}
\hline $\begin{array}{l}\text { Sub-bottom } \\
\text { depth } \\
\text { (m) }\end{array}$ & $\begin{array}{c}\delta^{18} \mathrm{O} \\
\left(\%_{00}\right)\end{array}$ & $\begin{array}{c}G . \\
\text { ruber } \\
(\%)\end{array}$ & $\begin{array}{c}G . \\
\text { bulloid. } \\
(\%)\end{array}$ & $\begin{array}{c}N . \\
\text { pachy. s. } \\
(\%)\end{array}$ & $\begin{array}{c}G . \\
\text { inflata } \\
(\%)\end{array}$ & $\begin{array}{l}\text { All } \\
\text { other } \\
(\%)\end{array}$ & $\begin{array}{l}\text { SSTw } \\
\left({ }^{\circ} \mathrm{C}\right)\end{array}$ \\
\hline 16.51 & $4.51 \mathrm{U}$ & & & & & & \\
\hline 16.51 & $2.36 \mathrm{C}$ & & & & & & \\
\hline 16.66 & $2.12 \mathrm{C}$ & 19.4 & 18.7 & 0.4 & 18.3 & 43.2 & 18.9 \\
\hline 16.81 & $2.65 \mathrm{C}$ & 11.9 & 21.1 & 1.4 & 24.2 & 41.4 & 16.7 \\
\hline 16.96 & $2.99 \mathrm{C}$ & 6.5 & 10.7 & 14.3 & 20.2 & 48.2 & 11.8 \\
\hline 17.03 & $3.17 \mathrm{C}$ & 1.0 & 7.3 & 49.7 & 4.9 & 37.1 & 5.8 \\
\hline 17.11 & $3.89 \mathrm{C}$ & & & & & & \\
\hline 17.26 & $4.10 \mathrm{C}$ & 1.9 & 19.0 & 10.3 & 28.7 & 40.2 & 9.8 \\
\hline 17.26 & $4.17 \mathrm{C}$ & & & & & & \\
\hline 17.33 & $4.18 \mathrm{C}$ & 1.8 & 19.6 & 7.8 & 11.1 & 59.6 & 9.7 \\
\hline 17.41 & & 1.0 & 13.9 & 18.4 & 17.1 & 49.7 & 8.4 \\
\hline 17.50 & & 1.0 & 21.6 & 27.7 & 9.9 & 39.7 & 6.3 \\
\hline 17.61 & $3.78 \mathrm{C}$ & & & & & & \\
\hline 17.63 & & 0.0 & 17.3 & 41.8 & 5.2 & 35.7 & 5.6 \\
\hline 17.71 & $3.60 \mathrm{C}$ & 0.9 & 25.9 & 16.4 & 8.6 & 48.1 & 6.5 \\
\hline 17.78 & & 1.1 & 25.6 & 19.3 & 9.6 & 44.4 & 6.3 \\
\hline 17.88 & $3.37 \mathrm{C}$ & 1.1 & 19.9 & 13.4 & 19.6 & 46.0 & 8.1 \\
\hline 17.88 & $4.74 \mathrm{U}$ & & & & & & \\
\hline 18.01 & $3.00 \mathrm{C}$ & 1.1 & 13.4 & 6.7 & 28.6 & 50.2 & 11.2 \\
\hline 18.16 & $4.74 \mathrm{U}$ & 2.1 & 10.1 & 5.1 & 20.3 & 62.4 & 12.3 \\
\hline 18.31 & $4.53 \mathrm{U}$ & 2.3 & 15.6 & 9.9 & 11.4 & 60.8 & 10.3 \\
\hline 18.31 & $3.24 \mathrm{C}$ & & & & & & \\
\hline 18.46 & $4.18 \mathrm{U}$ & 0.8 & 14.3 & 19.2 & 12.0 & 53.8 & 8.2 \\
\hline 18.46 & $3.99 \mathrm{C}$ & & & & & & \\
\hline 18.61 & $4.47 \mathrm{U}$ & 2.2 & 14.3 & 6.9 & 25.1 & 51.5 & 11.3 \\
\hline 18.61 & $3.75 \mathrm{C}$ & & & & & & \\
\hline 18.76 & $4.46 \mathrm{U}$ & 1.2 & 15.1 & 12.9 & 12.2 & 58.6 & 9.3 \\
\hline 18.84 & $3.63 \mathrm{U}$ & 0.0 & 19.0 & 4.8 & 11.2 & 65.0 & 9.8 \\
\hline 19.21 & $2.09 \mathrm{C}$ & 21.7 & 15.5 & 0.6 & 18.1 & 44.0 & 19.3 \\
\hline 19.36 & $2.57 \mathrm{C}$ & 19.6 & 13.3 & 1.5 & 21.1 & 44.4 & 19.0 \\
\hline 19.51 & $3.01 \mathrm{C}$ & 5.6 & 16.5 & 7.4 & 22.9 & 47.5 & 12.3 \\
\hline 19.73 & $3.37 \mathrm{C}$ & 0.3 & 13.8 & 39.9 & 4.5 & 41.5 & 6.5 \\
\hline 19.73 & $4.59 \mathrm{U}$ & & & & & & \\
\hline 19.81 & $3.64 \mathrm{C}$ & 1.5 & 20.4 & 7.9 & 19.4 & 50.8 & 9.4 \\
\hline 19.98 & $2.95 \mathrm{C}$ & 0.3 & 17.9 & 17.3 & 15.3 & 49.2 & 7.5 \\
\hline 20.11 & $3.15 \mathrm{C}$ & 1.5 & 18.3 & 17.9 & 8.4 & 53.8 & 8.1 \\
\hline 20.26 & $4.60 \mathrm{U}$ & 0.6 & 29.4 & 20.2 & 7.1 & 42.6 & 5.3 \\
\hline 20.26 & $3.72 \mathrm{C}$ & & & & & & \\
\hline 20.41 & $4.27 \mathrm{C}$ & 0.6 & 23.1 & 19.1 & 8.5 & 48.7 & 6.6 \\
\hline 20.56 & $4.80 \mathrm{U}$ & 1.9 & 19.1 & 7.4 & 20.1 & 51.5 & 10.0 \\
\hline 20.56 & $2.99 \mathrm{C}$ & & & & & & \\
\hline 20.56 & $4.24 \mathrm{U}$ & & & & & & \\
\hline 20.71 & $4.59 \mathrm{U}$ & 2.1 & 12.4 & 5.5 & 18.6 & 61.4 & 11.7 \\
\hline 20.71 & $3.39 \mathrm{C}$ & & & & & & \\
\hline 20.86 & $3.53 \mathrm{C}$ & 3.2 & 16.3 & 11.1 & 9.2 & 60.3 & 10.3 \\
\hline 20.86 & $4.56 \mathrm{U}$ & & & & & & \\
\hline 21.01 & $4.61 \mathrm{U}$ & 2.3 & 18.5 & 16.6 & 11.6 & 51.0 & 8.6 \\
\hline 21.01 & $3.94 \mathrm{C}$ & & & & & & \\
\hline 21.01 & $3.62 \mathrm{C}$ & & & & & & \\
\hline 21.01 & $4.53 \mathrm{U}$ & & & & & & \\
\hline 21.23 & $4.60 \mathrm{U}$ & 2.4 & 15.6 & 7.9 & 20.1 & 54.0 & 10.8 \\
\hline 20.23 & $3.46 \mathrm{C}$ & & & & & & \\
\hline 21.23 & $4.50 \mathrm{U}$ & & & & & & \\
\hline 21.31 & $4.35 \mathrm{U}$ & 0.2 & 16.9 & 14.3 & 9.4 & 59.4 & 8.4 \\
\hline 21.48 & $4.55 \mathrm{U}$ & 1.6 & 8.5 & 7.2 & 12.7 & 70.0 & 11.9 \\
\hline 21.48 & $3.12 \mathrm{C}$ & & & & & & \\
\hline 21.61 & $3.39 \mathrm{C}$ & 3.1 & 8.7 & 13.0 & 12.4 & 62.8 & 11.1 \\
\hline 21.61 & $4.22 \mathrm{U}$ & & & & & & \\
\hline 21.61 & $3.55 \mathrm{C}$ & & & & & & \\
\hline 21.76 & $4.35 \mathrm{U}$ & 0.7 & 8.1 & 16.1 & 16.5 & 58.6 & 9.6 \\
\hline 21.76 & $2.92 \mathrm{C}$ & & & & & & \\
\hline 21.76 & $4.37 \mathrm{U}$ & & & & & & \\
\hline 21.89 & $4.43 \mathrm{U}$ & 6.3 & 10.5 & 8.6 & 8.2 & 66.4 & 12.8 \\
\hline 21.91 & $4.10 \mathrm{U}$ & 2.5 & 10.2 & 4.9 & 13.0 & 69.4 & 12.4 \\
\hline 21.91 & $4.20 \mathrm{U}$ & & & & & & \\
\hline 22.06 & $2.84 \mathrm{C}$ & 4.1 & 9.0 & 4.5 & 23.2 & 59.2 & 13.5 \\
\hline 22.06 & $4.01 \mathrm{U}$ & & & & & & \\
\hline 22.06 & $4.01 \mathrm{U}$ & & & & & & \\
\hline 22.21 & $3.76 \mathrm{U}$ & 3.3 & 12.7 & 2.0 & 17.1 & 64.9 & 13.1 \\
\hline 22.36 & $3.52 \mathrm{U}$ & 6.8 & 8.6 & 1.1 & 18.4 & 65.0 & 15.2 \\
\hline 22.51 & $3.81 \mathrm{U}$ & 3.5 & 10.6 & 1.6 & 16.3 & 67.9 & 13.6 \\
\hline 22.51 & $3.20 \mathrm{C}$ & & & & & & \\
\hline 22.73 & $2.30 \mathrm{C}$ & 5.0 & 14.4 & 2.7 & 12.1 & 65.8 & 13.2 \\
\hline 22.81 & $3.41 \mathrm{C}$ & 4.9 & 16.2 & 9.2 & 13.7 & 56.1 & 11.3 \\
\hline 22.81 & $3.88 \mathrm{U}$ & & & & & & \\
\hline 22.88 & & 1.4 & 13.8 & 19.7 & 15.2 & 50.0 & 8.4 \\
\hline 22.98 & $3.34 \mathrm{C}$ & 2.5 & 8.7 & 9.4 & 23.9 & 55.4 & 11.6 \\
\hline 22.98 & $4.58 \mathrm{U}$ & & & & & & \\
\hline 23.11 & $4.53 \mathrm{U}$ & 4.0 & 14.4 & 1.4 & 21.6 & 58.5 & 13.4 \\
\hline 23.26 & $3.78 \mathrm{C}$ & 4.2 & 10.0 & 3.3 & 20.3 & 62.1 & 13.6 \\
\hline
\end{tabular}


Appendix C (continued).

\begin{tabular}{|c|c|c|c|c|c|c|c|}
\hline $\begin{array}{l}\text { Sub-bottom } \\
\text { depth } \\
\text { (m) }\end{array}$ & $\begin{array}{l}\delta^{18} \mathrm{O} \\
\left(\%_{0}\right)\end{array}$ & $\begin{array}{c}G . \\
\text { ruber } \\
(\%)\end{array}$ & $\begin{array}{c}\text { G. } \\
\text { bulloid. } \\
(\%)\end{array}$ & $\begin{array}{c}\text { N. } \\
\text { pachy. s. } \\
(\%)\end{array}$ & $\begin{array}{c}G . \\
\text { inflata } \\
(\%)\end{array}$ & $\begin{array}{l}\text { All } \\
\text { other } \\
(\%)\end{array}$ & $\begin{array}{l}\text { SSTw } \\
\left({ }^{\circ} \mathrm{C}\right)\end{array}$ \\
\hline 23.26 & $4.63 \mathrm{U}$ & & & & & & \\
\hline 23.41 & $3.43 \mathrm{C}$ & 2.4 & 8.8 & 2.1 & 25.5 & 61.1 & 13.6 \\
\hline 23.41 & $4.50 \mathrm{U}$ & & & & & & \\
\hline 23.48 & & 2.2 & 13.6 & 4.0 & 17.9 & 62.3 & 12.0 \\
\hline 23.56 & $2.92 \mathrm{C}$ & 0.7 & 13.2 & 3.7 & 20.7 & 61.7 & 11.5 \\
\hline 23.71 & $2.76 \mathrm{C}$ & 3.5 & 9.6 & 5.4 & 22.1 & 59.3 & 12.9 \\
\hline 23.71 & $3.33 \mathrm{C}$ & & & & & & \\
\hline 23.86 & $3.09 \mathrm{C}$ & 3.3 & 11.6 & 3.3 & 13.1 & 68.6 & 12.9 \\
\hline 23.86 & $2.95 \mathrm{C}$ & & & & & & \\
\hline 24.01 & $3.20 \mathrm{C}$ & 7.6 & 13.2 & 6.6 & 12.8 & 59.7 & 13.4 \\
\hline 24.01 & $2.91 \mathrm{C}$ & & & & & & \\
\hline 24.12 & & 5.8 & 9.6 & 12.9 & 8.3 & 63.4 & 12.0 \\
\hline 24.23 & $2.81 \mathrm{C}$ & 7.7 & 17.7 & 12.6 & 16.1 & 45.8 & 11.7 \\
\hline 24.31 & $2.65 \mathrm{C}$ & 2.8 & 14.2 & 4.5 & 14.2 & 64.2 & 11.9 \\
\hline 24.31 & $4.38 \mathrm{U}$ & & & & & & \\
\hline 24.39 & & 7.6 & 12.6 & 2.7 & 13.6 & 63.5 & 14.4 \\
\hline 24.48 & $2.44 \mathrm{C}$ & 4.5 & 12.0 & 3.4 & 12.4 & 67.8 & 13.2 \\
\hline 24.61 & $2.04 \mathrm{C}$ & 8.9 & 18.5 & 0.6 & 9.2 & 62.7 & 14.5 \\
\hline 25.21 & $2.81 \mathrm{C}$ & & & & & & \\
\hline 25.36 & $2.68 \mathrm{C}$ & & & & & & \\
\hline 25.51 & $2.70 \mathrm{C}$ & 7.0 & 10.0 & 3.2 & 10.2 & 69.7 & 14.4 \\
\hline 25.63 & & 4.5 & 9.6 & 1.6 & 14.1 & 70.2 & 14.0 \\
\hline 25.73 & $2.59 \mathrm{C}$ & 7.5 & 13.5 & 0.0 & 8.6 & 70.4 & 14.8 \\
\hline 25.81 & $2.94 \mathrm{C}$ & 4.6 & 9.9 & 1.3 & 10.2 & 74.0 & 14.1 \\
\hline 25.90 & & 5.2 & 14.7 & 1.4 & 10.1 & 68.6 & 13.5 \\
\hline 25.98 & $2.95 \mathrm{C}$ & 6.7 & 10.9 & 1.2 & 11.9 & 69.3 & 14.6 \\
\hline 25.98 & $2.87 \mathrm{C}$ & & & & & & \\
\hline 26.11 & $2.59 \mathrm{C}$ & 9.7 & 13.4 & 0.9 & 4.0 & 72.2 & 15.3 \\
\hline 26.18 & & 11.3 & 12.7 & 1.3 & 9.0 & 65.7 & 15.8 \\
\hline 26.26 & & 12.1 & 12.1 & 1.2 & 9.0 & 65.6 & 16.1 \\
\hline 26.31 & & 12.4 & 13.6 & 2.9 & 9.1 & 61.9 & 15.6 \\
\hline 26.41 & $2.90 \mathrm{C}$ & & & & & & \\
\hline 26.41 & $2.49 \mathrm{C}$ & 8.9 & 11.0 & 4.2 & 10.1 & 65.8 & 14.6 \\
\hline 26.44 & & $\begin{array}{l}0.9 \\
9.9\end{array}$ & 13.2 & 3.6 & 11.2 & 62.2 & $\begin{array}{l}14.0 \\
14.8\end{array}$ \\
\hline $\begin{array}{l}26.56 \\
26.71\end{array}$ & $2.81 \mathrm{C}$ & 12.5 & 12.5 & 5.9 & 12.5 & 56.6 & $\begin{array}{l}14.0 \\
15.1\end{array}$ \\
\hline $\begin{array}{l}26.71 \\
26.86\end{array}$ & $\begin{array}{l}2.81 \mathrm{C} \\
2.64 \mathrm{C}\end{array}$ & 9.8 & 17.5 & 5.8 & 12.7 & 54.2 & 13.8 \\
\hline $\begin{array}{l}26.86 \\
26.93\end{array}$ & & 11.6 & 13.5 & 7.6 & 15.3 & 52.0 & 14.5 \\
\hline $\begin{array}{l}26.93 \\
27.01\end{array}$ & $2.90 \mathrm{C}$ & 8.3 & 14.2 & 10.5 & 12.5 & 54.4 & 12.6 \\
\hline $\begin{array}{l}27.01 \\
27.12\end{array}$ & & 11.4 & 13.9 & 5.7 & 13.0 & 56.0 & 14.7 \\
\hline $\begin{array}{l}27.12 \\
27.23\end{array}$ & $2.81 \mathrm{C}$ & 9.7 & 13.0 & 8.8 & 12.7 & 55.8 & 13.6 \\
\hline 27.31 & & 7.9 & 15.1 & 9.2 & 11.0 & 56.8 & 12.6 \\
\hline 27.48 & $3.09 \mathrm{C}$ & 5.3 & 14.4 & 10.1 & 13.6 & 56.6 & 11.6 \\
\hline 27.61 & & 4.8 & 11.9 & 18.2 & 10.8 & 54.3 & 10.5 \\
\hline 27.76 & & 2.3 & 14.1 & 11.5 & 16.9 & 55.3 & 10.2 \\
\hline 27.91 & & 1.8 & 17.1 & 20.0 & 24.7 & 36.4 & 7.9 \\
\hline 28.06 & & 3.7 & 15.9 & 3.4 & 30.2 & 46.8 & 13.3 \\
\hline 28.21 & $4.03 \mathrm{C}$ & 0.7 & 15.2 & 2.8 & 31.1 & 50.2 & 12.1 \\
\hline 28.36 & & 3.3 & 18.5 & 2.7 & 35.8 & 39.7 & 14.1 \\
\hline 28.51 & $4.11 \mathrm{C}$ & 1.9 & 14.7 & 4.5 & 30.9 & 47.9 & 12.3 \\
\hline 28.73 & & 1.1 & 21.0 & 5.5 & 16.9 & 55.5 & 9.7 \\
\hline 28.81 & & 2.1 & 13.9 & 7.8 & 20.2 & 56.0 & 10.9 \\
\hline 28.98 & $4.16 \mathrm{C}$ & 1.0 & 16.7 & 6.2 & 27.9 & 48.2 & 10.7 \\
\hline 29.11 & $4.22 \mathrm{C}$ & 1.3 & 12.9 & 4.8 & 40.2 & 40.8 & 13.4 \\
\hline 29.26 & $3.59 \mathrm{C}$ & 2.4 & 20.0 & 4.8 & 29.0 & 43.8 & 11.5 \\
\hline 29.41 & $3.78 \mathrm{C}$ & 4.7 & 20.5 & 2.5 & 25.2 & 47.1 & 12.9 \\
\hline 29.56 & $3.65 \mathrm{C}$ & 2.7 & 14.2 & 7.5 & 20.7 & 54.9 & 11.2 \\
\hline 29.71 & $3.76 \mathrm{C}$ & 4.3 & 6.4 & 9.8 & 21.3 & 58.2 & 12.5 \\
\hline 29.86 & & 1.5 & 7.5 & 12.3 & 16.4 & 62.3 & 10.8 \\
\hline 30.01 & $3.21 \mathrm{C}$ & 1.8 & 7.5 & 12.6 & 12.9 & 65.3 & 10.9 \\
\hline 30.23 & & 2.2 & 18.2 & 16.0 & 21.2 & 42.5 & 8.5 \\
\hline 30.31 & $4.44 \mathrm{U}$ & 2.2 & 9.3 & 28.5 & 16.1 & 44.0 & 8.2 \\
\hline 30.33 & $3.27 \mathrm{C}$ & & & & & & \\
\hline 30.48 & $3.24 \mathrm{C}$ & 3.6 & 13.0 & 15.1 & 17.2 & 51.1 & 10.2 \\
\hline 30.61 & $3.29 \mathrm{C}$ & 4.8 & 16.1 & 10.6 & 11.9 & 56.6 & 11.0 \\
\hline 30.76 & $3.41 \mathrm{C}$ & 3.3 & 8.1 & 19.5 & 7.2 & 61.9 & 10.3 \\
\hline 30.91 & $3.23 \mathrm{C}$ & 4.9 & 17.9 & 8.5 & 13.5 & 55.2 & 11.2 \\
\hline 31.06 & $2.37 \mathrm{C}$ & 11.7 & 17.5 & 0.4 & 23.0 & 47.4 & 16.8 \\
\hline 31.21 & $2.73 \mathrm{C}$ & 17.5 & 11.7 & 4.4 & 13.9 & 52.6 & 16.9 \\
\hline 31.36 & $3.11 \mathrm{C}$ & 14.9 & 11.7 & 0.0 & 19.2 & 54.2 & 18.0 \\
\hline 31.51 & $2.78 \mathrm{C}$ & 11.1 & 16.9 & 2.0 & 16.9 & 53.1 & 15.5 \\
\hline 31.51 & $2.79 \mathrm{C}$ & & & & & & \\
\hline 31.62 & & 20.4 & 11.8 & 1.1 & 10.5 & 56.3 & 18.2 \\
\hline 31.73 & $2.96 \mathrm{C}$ & 17.2 & 12.8 & 0.9 & 10.3 & 58.7 & 17.5 \\
\hline 31.81 & $2.86 \mathrm{C}$ & 15.1 & 11.1 & 2.7 & 17.1 & 54.0 & 17.1 \\
\hline 31.98 & $3.93 \mathrm{U}$ & 5.3 & 14.7 & 37.9 & 12.6 & 29.5 & 8.0 \\
\hline 31.98 & $2.79 \mathrm{C}$ & & & & & & \\
\hline 32.11 & $4.38 \mathrm{U}$ & 0.0 & 4.7 & 72.4 & 11.5 & 11.5 & 0.4 \\
\hline 32.11 & $3.53 \mathrm{C}$ & & & & & & \\
\hline 32.26 & $4.53 \mathrm{U}$ & 2.3 & 7.4 & 26.1 & 27.6 & 36.6 & 8.0 \\
\hline 32.41 & $3.16 \mathrm{C}$ & 0.9 & 8.9 & 27.0 & 21.7 & 41.5 & 7.5 \\
\hline 32.41 & $4.57 \mathrm{U}$ & & & & & & \\
\hline
\end{tabular}

Appendix C (continued).

\begin{tabular}{|c|c|c|c|c|c|c|c|}
\hline $\begin{array}{l}\text { Sub-bottom } \\
\text { depth } \\
\text { (m) }\end{array}$ & $\begin{array}{l}\delta^{18} \mathrm{O} \\
\left(\psi_{0}\right)\end{array}$ & $\begin{array}{c}G . \\
\text { ruber } \\
(\%)\end{array}$ & $\begin{array}{c}\text { G. } \\
\text { bulloid. } \\
(\%)\end{array}$ & $\begin{array}{c}\text { N. } \\
\text { pachy. s. } \\
(\%)\end{array}$ & $\begin{array}{c}G . \\
\text { inflata } \\
(\%)\end{array}$ & $\begin{array}{c}\text { All } \\
\text { other } \\
(\%)\end{array}$ & $\begin{array}{l}\text { SSTw } \\
\left({ }^{\circ} \mathrm{C}\right)\end{array}$ \\
\hline 32.56 & $4.44 \mathrm{U}$ & 2.2 & 9.5 & 12.0 & 29.5 & 46.9 & 10.9 \\
\hline 32.71 & $3.57 \mathrm{C}$ & 1.4 & 9.8 & 10.3 & 24.6 & 53.9 & 10.8 \\
\hline 32.71 & $4.42 \mathrm{U}$ & & & & & & \\
\hline 32.86 & $3.51 \mathrm{C}$ & 2.4 & 15.6 & 4.6 & 18.7 & 58.7 & 11.6 \\
\hline 32.86 & $4.05 \mathrm{U}$ & & & & & & \\
\hline 33.01 & $3.11 \mathrm{C}$ & 4.3 & 18.1 & 5.7 & 8.2 & 63.7 & 11.6 \\
\hline 33.23 & $3.35 \mathrm{C}$ & 8.3 & 13.3 & 4.1 & 12.7 & 61.6 & 14.1 \\
\hline 33.31 & $1.16 \mathrm{U}$ & 4.1 & 13.5 & 3.8 & 16.2 & 62.4 & 12.8 \\
\hline 33.31 & $3.26 \mathrm{C}$ & & & & & & \\
\hline 33.48 & $4.51 \mathrm{U}$ & 4.2 & 15.2 & 14.2 & 17.7 & 48.6 & 10.3 \\
\hline 33.48 & $3.02 \mathrm{C}$ & & & & & & \\
\hline 33.61 & $2.75 \mathrm{C}$ & 2.6 & 9.6 & 10.7 & 20.2 & 57.0 & 11.2 \\
\hline 33.61 & $4.47 \mathrm{U}$ & & & & & & \\
\hline 33.76 & $3.66 \mathrm{C}$ & 2.5 & 14.4 & 19.5 & 14.4 & 49.0 & 8.9 \\
\hline 33.76 & $4.55 \mathrm{U}$ & & & & & & \\
\hline 33.91 & $4.53 \mathrm{U}$ & 0.3 & 6.8 & 10.5 & 18.3 & 64.1 & 10.8 \\
\hline 34.06 & $4.48 \mathrm{U}$ & 0.6 & 13.5 & 7.4 & 26.4 & 52.1 & 10.6 \\
\hline 34.21 & $4.37 \mathrm{U}$ & 1.5 & 7.9 & 6.2 & 16.4 & 68.0 & 12.1 \\
\hline 34.36 & $4.32 \mathrm{U}$ & 3.1 & 9.3 & 11.1 & 34.9 & 41.5 & 12.0 \\
\hline 34.44 & $4.20 \mathrm{U}$ & 3.4 & 16.7 & 8.2 & 23.4 & 48.3 & 11.1 \\
\hline 34.81 & & 1.6 & 8.4 & 11.3 & 20.4 & 58.3 & 10.8 \\
\hline 34.96 & $3.18 \mathrm{C}$ & 1.5 & 13.6 & 12.1 & 22.4 & 50.3 & 9.8 \\
\hline 35.02 & $3.68 \mathrm{C}$ & 2.4 & 24.4 & 11.7 & 16.2 & 45.4 & 8.2 \\
\hline 35.11 & $3.22 \mathrm{C}$ & 2.5 & 13.2 & 5.1 & 12.4 & 66.9 & 11.9 \\
\hline 35.33 & $3.60 \mathrm{C}$ & 5.7 & 10.9 & 12.0 & 20.3 & 51.0 & 12.0 \\
\hline 35.41 & $3.55 \mathrm{C}$ & 3.4 & 16.7 & 17.3 & 16.1 & 46.4 & 9.2 \\
\hline 35.58 & $2.72 \mathrm{C}$ & 3.3 & 12.0 & 4.3 & 27.7 & 52.7 & 13.1 \\
\hline 35.73 & $3.09 \mathrm{C}$ & 3.8 & 9.5 & 21.0 & 12.1 & 53.7 & 10.0 \\
\hline 35.86 & $3.10 \mathrm{C}$ & 5.9 & 10.2 & 13.8 & 11.5 & 58.6 & 11.7 \\
\hline 35.99 & $2.71 \mathrm{C}$ & 7.1 & 14.9 & 7.5 & 18.8 & 51.6 & 12.9 \\
\hline 36.16 & $2.97 \mathrm{C}$ & 5.8 & 16.0 & 6.1 & 14.1 & 58.0 & 12.4 \\
\hline 36.31 & $2.52 \mathrm{C}$ & 7.0 & 13.3 & 11.1 & 9.2 & 59.5 & 12.2 \\
\hline 36.46 & $2.57 \mathrm{C}$ & 4.4 & 14.5 & 11.6 & 11.3 & 58.2 & 10.9 \\
\hline 36.46 & $3.63 \mathrm{U}$ & & & & & & \\
\hline 36.61 & $3.23 \mathrm{C}$ & 11.2 & 8.4 & 3.7 & 15.9 & 60.8 & 15.9 \\
\hline 36.67 & & 13.0 & 10.9 & 8.3 & 14.2 & 53.7 & 14.9 \\
\hline 36.76 & & 8.5 & 8.8 & 24.4 & 7.9 & 50.3 & 11.3 \\
\hline 36.83 & $3.16 \mathrm{C}$ & 4.0 & 12.6 & 35.6 & 9.8 & 38.0 & 8.2 \\
\hline 36.91 & $2.92 \mathrm{C}$ & 0.4 & 3.3 & 75.9 & 3.3 & 17.2 & 1.3 \\
\hline 37.08 & $1.96 \mathrm{C}$ & 1.5 & 5.5 & 49.1 & 16.8 & 27.1 & 4.2 \\
\hline 37.21 & & 0.3 & 4.1 & 50.7 & 19.5 & 25.4 & 3.0 \\
\hline 37.37 & & 0.4 & 10.8 & 39.2 & 16.2 & 33.5 & 5.6 \\
\hline 37.51 & $3.55 \mathrm{C}$ & 1.8 & 11.6 & 45.1 & 9.4 & 32.1 & 6.0 \\
\hline 37.66 & $3.78 \mathrm{C}$ & 0.6 & 5.4 & 59.5 & 11.4 & 23.1 & 2.7 \\
\hline 37.81 & & 0.0 & 4.8 & 82.9 & 0.6 & 11.6 & 0.9 \\
\hline 37.98 & & 0.6 & 4.0 & 60.3 & 12.9 & 22.1 & 2.3 \\
\hline 38.11 & & 0.7 & 6.8 & 30.0 & 19.8 & 42.7 & 7.3 \\
\hline 38.17 & & 2.2 & 6.2 & 23.7 & 15.3 & 52.6 & 9.2 \\
\hline 38.33 & $3.16 \mathrm{C}$ & 1.6 & 8.8 & 4.5 & 14.6 & 70.5 & 12.4 \\
\hline 38.41 & & 2.2 & 9.9 & 8.7 & 20.4 & 58.8 & 11.4 \\
\hline 38.58 & $3.05 \mathrm{C}$ & 2.2 & 8.7 & 9.9 & 19.2 & 60.1 & 11.3 \\
\hline 38.73 & $2.58 \mathrm{C}$ & 2.6 & 7.1 & 19.4 & 9.7 & 61.2 & 10.1 \\
\hline 38.86 & $3.14 \mathrm{C}$ & 1.9 & 9.6 & 6.4 & 9.2 & 72.9 & 12.0 \\
\hline 39.01 & $3.30 \mathrm{C}$ & 5.2 & 11.9 & 13.3 & 14.1 & 55.6 & 11.3 \\
\hline 39.16 & $3.37 \mathrm{C}$ & 1.9 & 6.9 & 49.1 & 7.2 & 35.0 & 5.9 \\
\hline 39.23 & & 0.0 & 4.9 & 61.9 & 8.1 & 25.1 & 2.7 \\
\hline 39.31 & $4.48 \mathrm{U}$ & 0.0 & 1.7 & 44.9 & 21.1 & 32.3 & 4.2 \\
\hline 39.39 & & 0.0 & 3.4 & 28.4 & 19.8 & 48.5 & 7.6 \\
\hline 39.46 & & 0.6 & 7.0 & 31.9 & 13.1 & 47.3 & 7.5 \\
\hline 39.61 & & 3.6 & 6.4 & 21.0 & 20.2 & 48.8 & 10.0 \\
\hline 39.67 & & 3.0 & 10.1 & 39.8 & 16.9 & 30.3 & 6.5 \\
\hline 39.76 & & 4.2 & 16.6 & 29.0 & 10.3 & 39.9 & 8.6 \\
\hline 39.83 & $3.35 \mathrm{C}$ & 3.8 & 8.2 & 38.6 & 11.4 & 38.0 & 7.8 \\
\hline 39.83 & $2.98 \mathrm{C}$ & & & & & & \\
\hline 39.89 & $3.29 \mathrm{C}$ & 6.3 & 8.9 & 30.0 & 15.1 & 39.7 & 9.6 \\
\hline 39.98 & $3.18 \mathrm{C}$ & 3.3 & 10.3 & 20.0 & 13.6 & 52.7 & 9.8 \\
\hline 40.03 & & 5.3 & 19.8 & 17.2 & 11.2 & 46.5 & 9.6 \\
\hline 40.08 & & 4.7 & 10.3 & 10.0 & 15.3 & 59.7 & 12.0 \\
\hline 40.21 & $2.34 \mathrm{C}$ & 2.5 & 8.8 & 17.5 & 16.6 & 54.5 & 10.0 \\
\hline 40.36 & $2.68 \mathrm{C}$ & 7.8 & 6.6 & 3.6 & 17.7 & 64.4 & 15.1 \\
\hline 40.51 & $2.69 \mathrm{C}$ & 7.6 & 10.9 & 1.8 & 12.0 & 67.6 & 14.8 \\
\hline 40.61 & $2.55 \mathrm{C}$ & 6.1 & 11.6 & 2.6 & 19.0 & 60.6 & 14.2 \\
\hline 40.83 & $2.55 \mathrm{C}$ & 12.3 & 14.1 & 4.6 & 16.3 & 52.8 & 15.4 \\
\hline 40.96 & $1.98 \mathrm{C}$ & 13.1 & 11.4 & 7.3 & 15.2 & 52.9 & 15.2 \\
\hline 41.09 & $1.96 \mathrm{C}$ & 8.6 & 22.4 & 23.4 & 14.5 & 31.0 & 10.4 \\
\hline 41.17 & $4.48 \mathrm{U}$ & 2.5 & 19.9 & 28.4 & 4.3 & 45.0 & 7.6 \\
\hline 41.26 & & 3.8 & 9.0 & 28.3 & 14.7 & 44.2 & 9.0 \\
\hline 41.33 & $4.42 \mathrm{U}$ & 0.9 & 9.1 & 38.4 & 8.4 & 43.1 & 7.0 \\
\hline 41.41 & & 7.8 & 16.7 & 3.7 & 10.7 & 61.2 & 13.6 \\
\hline 41.58 & $3.45 \mathrm{C}$ & 1.8 & 13.1 & 3.5 & 14.9 & 66.7 & 12.0 \\
\hline 41.58 & $4.09 \mathrm{U}$ & & & & & & \\
\hline 41.71 & $3.84 \mathrm{U}$ & 5.4 & 14.0 & 6.0 & 18.7 & 55.9 & 12.7 \\
\hline
\end{tabular}


Appendix C (continued).

\begin{tabular}{|c|c|c|c|c|c|c|c|}
\hline $\begin{array}{l}\text { Sub-bottom } \\
\text { depth } \\
\text { (m) }\end{array}$ & $\begin{array}{l}\delta^{18} \mathrm{O} \\
\left(\%\left({ }_{0}\right)\right.\end{array}$ & $\begin{array}{c}G . \\
\text { ruber } \\
(\%)\end{array}$ & $\begin{array}{c}\text { G. } \\
\text { bulloid. } \\
(\%)\end{array}$ & $\begin{array}{c}N . \\
\text { pachy. s. } \\
(\%)\end{array}$ & $\begin{array}{c}G . \\
\text { inflata } \\
(\%)\end{array}$ & $\begin{array}{c}\text { All } \\
\text { other } \\
(\%)\end{array}$ & $\begin{array}{l}\text { SSTw } \\
\left({ }^{\circ} \mathrm{C}\right)\end{array}$ \\
\hline 41.86 & $3.74 \mathrm{U}$ & 5.2 & 12.6 & 4.8 & 11.3 & 66.1 & 13.0 \\
\hline 41.86 & $2.69 \mathrm{C}$ & & & & & & \\
\hline 41.99 & & 2.3 & 13.8 & 16.4 & 6.4 & 61.1 & 9.6 \\
\hline 42.07 & & 6.1 & 11.2 & 15.1 & 12.5 & 55.1 & 11.4 \\
\hline 42.16 & $2.94 \mathrm{C}$ & 1.8 & 10.7 & 22.7 & 8.7 & 56.1 & 8.9 \\
\hline 42.16 & $4.04 \mathrm{U}$ & & & & & & \\
\hline 42.31 & $3.31 \mathrm{C}$ & 3.3 & 3.3 & 13.9 & 16.2 & 63.2 & 11.7 \\
\hline 42.31 & $4.14 \mathrm{U}$ & & & & & & \\
\hline 42.46 & & 2.6 & 6.7 & 5.4 & 26.6 & 58.7 & 13.1 \\
\hline 42.61 & $3.16 \mathrm{C}$ & 5.9 & 9.4 & 3.8 & 15.7 & 65.2 & 14.0 \\
\hline 42.66 & $2.97 \mathrm{C}$ & 5.5 & 11.0 & 5.8 & 15.0 & 62.7 & 13.1 \\
\hline 42.83 & $2.97 \mathrm{C}$ & 2.9 & 13.1 & 8.7 & 20.2 & 55.1 & 11.2 \\
\hline 42.91 & $2.92 \mathrm{C}$ & 3.9 & 10.5 & 25.8 & 5.8 & 54.0 & 9.5 \\
\hline 43.08 & $3.47 \mathrm{C}$ & 1.4 & 8.1 & 50.7 & 5.5 & 34.2 & 5.6 \\
\hline 43.18 & $3.31 \mathrm{C}$ & 6.0 & 9.1 & 34.9 & 11.0 & 39.0 & 9.1 \\
\hline 43.36 & $1.85 \mathrm{C}$ & 6.3 & 8.5 & 28.8 & 11.0 & 45.5 & 10.0 \\
\hline 43.51 & $3.08 \mathrm{C}$ & 5.5 & 6.1 & 39.9 & 11.7 & 36.8 & 8.2 \\
\hline 43.66 & & 8.5 & 11.8 & 9.9 & 18.7 & 51.1 & 13.3 \\
\hline 43.81 & $3.03 \mathrm{C}$ & 5.8 & 8.8 & 18.8 & 15.4 & 51.3 & 11.0 \\
\hline 43.96 & $2.57 \mathrm{C}$ & 12.3 & 8.3 & 4.3 & 17.4 & 57.6 & 16.1 \\
\hline 44.01 & & 13.8 & 12.4 & 3.2 & 12.9 & 57.6 & 16.2 \\
\hline 44.41 & $2.71 \mathrm{C}$ & 3.6 & 10.5 & 36.5 & 5.3 & 44.1 & 8.4 \\
\hline 44.56 & $3.47 \mathrm{C}$ & 4.4 & 10.3 & 36.3 & 10.6 & 38.4 & 8.4 \\
\hline 44.71 & $3.38 \mathrm{C}$ & 3.1 & 14.5 & 29.0 & 13.4 & 40.0 & 8.1 \\
\hline 44.93 & $2.45 \mathrm{C}$ & 3.3 & 11.2 & 32.0 & 8.2 & 45.3 & 8.6 \\
\hline 45.01 & $4.41 \mathrm{U}$ & 1.5 & 12.5 & 4.9 & 6.1 & 74.9 & 11.8 \\
\hline 45.10 & $3.22 \mathrm{U}$ & 14.1 & 10.6 & 0.5 & 22.3 & 52.4 & 18.0 \\
\hline 45.10 & $2.42 \mathrm{C}$ & & & & & & \\
\hline 45.18 & $2.53 \mathrm{C}$ & 9.6 & 13.3 & 1.1 & 14.8 & 61.3 & 15.4 \\
\hline 45.18 & $3.12 \mathrm{U}$ & & & & & & \\
\hline 45.18 & $2.64 \mathrm{C}$ & & & & & & \\
\hline 45.31 & $2.25 \mathrm{C}$ & 17.7 & 4.8 & 2.3 & 12.9 & 62.3 & 17.9 \\
\hline 45.46 & $2.48 \mathrm{C}$ & 21.4 & 7.4 & 0.0 & 14.4 & 56.8 & 19.2 \\
\hline 45.61 & $2.54 \mathrm{C}$ & 22.3 & 5.0 & 1.7 & 9.0 & 62.0 & 18.7 \\
\hline 45.76 & $2.36 \mathrm{C}$ & 20.8 & 8.1 & 0.0 & 7.8 & 63.4 & 18.7 \\
\hline 45.91 & $2.54 \mathrm{C}$ & 22.9 & 9.1 & 3.1 & 18.6 & 46.3 & 18.6 \\
\hline 46.06 & $2.70 \mathrm{C}$ & 14.5 & 16.5 & 1.2 & 17.3 & 50.6 & 17.0 \\
\hline 46.06 & $3.05 \mathrm{C}$ & & & & & & \\
\hline 46.21 & $2.93 \mathrm{C}$ & 10.3 & 10.3 & 16.1 & 19.7 & 43.6 & 12.8 \\
\hline 46.29 & $3.11 \mathrm{C}$ & 8.5 & 17.3 & 26.6 & 14.6 & 33.0 & 10.4 \\
\hline 46.43 & $3.59 \mathrm{U}$ & 5.6 & 9.4 & 4.9 & 22.3 & 57.8 & 13.9 \\
\hline 46.51 & $2.62 \mathrm{C}$ & 6.8 & 4.5 & 23.4 & 20.1 & 45.1 & 10.8 \\
\hline 46.68 & $2.82 \mathrm{C}$ & 12.1 & 11.8 & 2.4 & 20.5 & 53.2 & 16.5 \\
\hline 46.79 & $2.79 \mathrm{C}$ & 12.7 & 12.6 & 6.5 & 10.9 & 57.4 & 15.0 \\
\hline 46.96 & $3.19 \mathrm{C}$ & 13.3 & 10.4 & 2.9 & 10.7 & 62.8 & 16.3 \\
\hline 46.96 & $2.88 \mathrm{C}$ & & & & & & \\
\hline 47.11 & $3.25 \mathrm{C}$ & 7.1 & 10.2 & 8.1 & 18.3 & 56.3 & 13.4 \\
\hline 47.26 & & 4.0 & 11.2 & 24.5 & 14.9 & 45.5 & 9.3 \\
\hline 47.41 & & 1.9 & 5.3 & 9.7 & 30.5 & 52.5 & 12.0 \\
\hline 47.44 & & 4.7 & 6.8 & 9.4 & 28.8 & 50.3 & 13.0 \\
\hline 47.48 & $3.51 \mathrm{C}$ & 3.4 & 6.8 & 11.5 & 28.3 & 50.0 & 11.9 \\
\hline 47.56 & $3.10 \mathrm{C}$ & 2.5 & 5.5 & 16.8 & 23.4 & 51.8 & 10.4 \\
\hline 47.63 & $3.34 \mathrm{C}$ & 5.2 & 9.5 & 23.6 & 25.9 & 35.7 & 9.6 \\
\hline 47.71 & $3.36 \mathrm{C}$ & 2.9 & 5.2 & 39.8 & 14.6 & 37.5 & 7.1 \\
\hline 47.80 & $3.19 \mathrm{C}$ & 6.3 & 10.1 & 20.6 & 18.6 & 44.3 & 10.7 \\
\hline 47.93 & $2.97 \mathrm{C}$ & 4.7 & 9.4 & 20.3 & 15.2 & 50.4 & 10.3 \\
\hline 48.03 & $3.14 \mathrm{C}$ & 9.5 & 8.4 & 10.2 & 31.9 & 40.0 & 14.8 \\
\hline 48.18 & $3.67 \mathrm{U}$ & 8.5 & 7.7 & 10.7 & 15.6 & 57.5 & 13.5 \\
\hline 48.31 & $2.76 \mathrm{C}$ & 11.6 & 6.8 & 7.1 & 15.3 & 59.2 & 15.2 \\
\hline 48.41 & & 13.6 & 11.6 & 7.9 & 8.3 & 58.6 & 15.0 \\
\hline 48.43 & & 16.6 & 12.8 & 7.3 & 6.2 & 57.1 & 15.7 \\
\hline 48.46 & $2.58 \mathrm{C}$ & 14.2 & 9.1 & 4.7 & 10.7 & 61.2 & 16.1 \\
\hline 48.61 & $2.81 \mathrm{C}$ & 11.7 & 11.4 & 3.9 & 8.8 & 64.2 & 15.4 \\
\hline 48.69 & $2.73 \mathrm{C}$ & 18.3 & 14.3 & 2.7 & 9.1 & 55.5 & 17.1 \\
\hline 48.76 & $2.73 \mathrm{C}$ & 12.8 & 14.1 & 4.2 & 11.2 & 57.7 & 15.4 \\
\hline 48.91 & $2.75 \mathrm{C}$ & 18.4 & 10.2 & 3.0 & 6.3 & 62.0 & 17.3 \\
\hline 49.06 & $2.77 \mathrm{C}$ & 15.5 & 14.5 & 0.3 & 13.2 & 56.6 & 17.3 \\
\hline 49.19 & $2.85 \mathrm{C}$ & 11.3 & 14.1 & 10.8 & 17.2 & 46.7 & 13.8 \\
\hline 49.30 & & 20.1 & 15.0 & 0.8 & 2.8 & 61.4 & 17.8 \\
\hline 49.43 & $2.67 \mathrm{C}$ & 9.1 & 17.8 & 2.2 & 14.1 & 56.9 & 14.4 \\
\hline 49.51 & $2.72 \mathrm{C}$ & 17.1 & 11.6 & 2.6 & 10.7 & 58.1 & 17.1 \\
\hline 49.68 & $2.79 \mathrm{C}$ & 15.4 & 15.1 & 0.6 & 6.8 & 62.1 & 16.8 \\
\hline 49.81 & $3.45 \mathrm{C}$ & 14.4 & 10.8 & 4.9 & 4.2 & 65.7 & 15.9 \\
\hline 49.85 & $2.90 \mathrm{C}$ & 14.9 & 13.3 & 6.5 & 4.9 & 60.3 & 15.4 \\
\hline 49.96 & & 6.4 & 7.3 & 14.9 & 4.7 & 66.7 & 12.2 \\
\hline 50.09 & $4.16 \mathrm{U}$ & & & & & & \\
\hline 50.11 & $4.19 \mathrm{U}$ & 5.9 & 6.8 & 7.4 & 13.9 & 66.1 & 13.5 \\
\hline 50.26 & $4.43 \mathrm{U}$ & 6.0 & 5.3 & 10.3 & 12.6 & 65.9 & 13.1 \\
\hline 50.41 & $3.86 \mathrm{C}$ & 5.5 & 3.4 & 7.9 & 7.6 & 75.5 & 13.7 \\
\hline 50.41 & $4.36 \mathrm{U}$ & & & & & & \\
\hline 50.48 & $4.31 \mathrm{U}$ & 4.2 & 7.7 & 4.5 & 26.8 & 56.8 & 13.9 \\
\hline 50.56 & $3.41 \mathrm{C}$ & 8.0 & 8.7 & 3.1 & 13.9 & 66.2 & 14.9 \\
\hline
\end{tabular}

Appendix C (continued).

\begin{tabular}{|c|c|c|c|c|c|c|c|}
\hline $\begin{array}{l}\text { Sub-bottom } \\
\text { depth } \\
\text { (m) }\end{array}$ & $\begin{array}{l}\delta^{18} \mathrm{O} \\
\left(\%_{00}\right)\end{array}$ & $\begin{array}{l}G . \\
\text { ruber } \\
(\%)\end{array}$ & $\begin{array}{c}G . \\
\text { bulloid. } \\
(\%)\end{array}$ & $\begin{array}{c}\text { N. } \\
\text { pachy, s. } \\
(\%)\end{array}$ & $\begin{array}{c}G . \\
\text { inflata } \\
(\%)\end{array}$ & $\begin{array}{l}\text { All } \\
\text { other } \\
(\%)\end{array}$ & $\begin{array}{l}\text { SSTw } \\
\left({ }^{\circ} \mathrm{C}\right)\end{array}$ \\
\hline 50.58 & & 8.9 & 8.6 & 5.1 & 16.8 & 60.6 & 14.8 \\
\hline 50.71 & $3.62 \mathrm{C}$ & 5.9 & 6.7 & 2.6 & 23.4 & 61.3 & 15.0 \\
\hline 50.78 & & 10.3 & 10.7 & 0.7 & 10.3 & 68.0 & 15.9 \\
\hline 50.93 & $4.15 \mathrm{U}$ & 13.5 & 5.3 & 2.3 & 22.0 & 56.9 & 17.5 \\
\hline 51.00 & & 11.8 & 8.9 & 1.0 & 23.6 & 54.6 & 17.3 \\
\hline 51.01 & $4.06 \mathrm{U}$ & 13.7 & 4.3 & 2.2 & 20.5 & 59.3 & 17.5 \\
\hline 51.01 & $3.19 \mathrm{C}$ & & & & & & \\
\hline 51.18 & $3.88 \mathrm{U}$ & 8.4 & 7.2 & 0.0 & 16.4 & 68.0 & 16.1 \\
\hline 51.18 & $3.03 \mathrm{C}$ & & & & & & \\
\hline 51.31 & $2.16 \mathrm{C}$ & 7.2 & 7.6 & 2.0 & 16.1 & 67.1 & 15.2 \\
\hline 51.46 & $2.40 \mathrm{C}$ & 22.1 & 13.1 & 0.0 & 23.2 & 41.5 & 20.4 \\
\hline 51.61 & $2.55 \mathrm{C}$ & 14.8 & 11.7 & 0.8 & 19.1 & 53.5 & 17.7 \\
\hline 51.76 & & 18.7 & 14.0 & 0.3 & 15.7 & 51.3 & 18.4 \\
\hline 51.91 & & 10.2 & 10.2 & 0.7 & 21.1 & 57.8 & 16.5 \\
\hline 52.06 & $2.66 \mathrm{C}$ & 7.0 & 10.4 & 1.2 & 19.5 & 61.9 & 15.1 \\
\hline 52.21 & $4.36 \mathrm{U}$ & 5.6 & 4.0 & 1.6 & 26.7 & 62.2 & 15.7 \\
\hline 52.28 & $4.43 \mathrm{U}$ & 8.5 & 9.7 & 0.3 & 13.9 & 67.6 & 15.7 \\
\hline 52.43 & $4.51 \mathrm{U}$ & 0.9 & 7.2 & 1.2 & 16.2 & 74.5 & 13.3 \\
\hline 52.51 & $4.48 \mathrm{U}$ & 1.4 & 2.8 & 0.0 & 31.2 & 64.5 & 15.0 \\
\hline 52.60 & & 3.5 & 8.9 & 0.7 & 16.1 & 70.9 & 14.1 \\
\hline 52.68 & $2.95 \mathrm{C}$ & 5.8 & 7.2 & 0.3 & 18.6 & 68.1 & 15.3 \\
\hline 52.81 & $3.41 \mathrm{C}$ & 6.5 & 6.2 & 4.9 & 15.4 & 67.0 & 14.4 \\
\hline 52.96 & & 16.9 & 7.1 & 0.3 & 15.4 & 60.3 & 18.3 \\
\hline 53.09 & & 10.6 & 8.8 & 0.3 & 27.3 & 53.0 & 17.6 \\
\hline 53.26 & $2.91 \mathrm{C}$ & 21.8 & 16.1 & 0.4 & 18.8 & 42.9 & 19.5 \\
\hline 53.41 & $2.73 \mathrm{C}$ & 15.3 & 11.7 & 0.3 & 17.3 & 55.3 & 17.8 \\
\hline 53.49 & $2.67 \mathrm{C}$ & 14.2 & 11.9 & 1.6 & 29.0 & 43.2 & 18.6 \\
\hline 54.05 & & 4.6 & 11.1 & 0.9 & 25.6 & 57.7 & 14.6 \\
\hline 54.16 & $3.64 \mathrm{U}$ & 10.0 & 10.3 & 0.7 & 18.9 & 60.0 & 16.3 \\
\hline 54.29 & $3.65 \mathrm{U}$ & 7.5 & 14.2 & 1.1 & 28.1 & 49.1 & 15.7 \\
\hline 54.37 & $2.86 \mathrm{C}$ & 12.6 & 12.1 & 0.0 & 22.7 & 52.6 & 17.6 \\
\hline 54.53 & & 15.6 & 10.1 & 0.0 & 16.7 & 57.6 & 18.0 \\
\hline 54.61 & $2.68 \mathrm{C}$ & 15.6 & 12.6 & 1.7 & 11.6 & 58.5 & 17.0 \\
\hline 54.78 & $3.30 \mathrm{C}$ & 12.7 & 11.3 & 0.0 & 8.0 & 68.0 & 16.7 \\
\hline 54.91 & $3.88 \mathrm{U}$ & 5.7 & 13.0 & 0.4 & 24.0 & 56.9 & 14.9 \\
\hline 55.06 & $4.00 \mathrm{U}$ & 7.0 & 13.4 & 0.0 & 24.3 & 55.3 & 15.5 \\
\hline 55.21 & & 6.5 & 16.4 & 0.3 & 15.0 & 61.8 & 14.1 \\
\hline 55.36 & & 7.3 & 10.1 & 0.6 & 19.0 & 63.0 & 15.4 \\
\hline 55.51 & $3.14 \mathrm{C}$ & 10.2 & 10.2 & 1.2 & 0.3 & 78.1 & 15.7 \\
\hline 55.66 & & 2.5 & 10.1 & 1.2 & 28.7 & 57.5 & 13.9 \\
\hline 55.74 & & 2.7 & 12.2 & 1.0 & 25.8 & 58.3 & 13.6 \\
\hline 55.81 & $4.26 \mathrm{U}$ & 5.0 & 9.2 & 0.4 & 38.3 & 47.1 & 16.8 \\
\hline 55.87 & $4.09 \mathrm{U}$ & 6.4 & 11.6 & 1.5 & 27.1 & 53.4 & 15.3 \\
\hline 56.03 & $3.42 \mathrm{U}$ & 19.4 & 7.7 & 0.7 & 14.3 & 57.9 & 18.6 \\
\hline 56.03 & $2.92 \mathrm{C}$ & & & & & & \\
\hline 56.13 & $3.57 \mathrm{U}$ & 16.4 & 9.4 & 1.0 & 14.0 & 59.1 & 17.8 \\
\hline 56.28 & $2.63 \mathrm{C}$ & 13.1 & 10.5 & 1.3 & 15.6 & 59.6 & 16.8 \\
\hline 56.41 & $2.08 \mathrm{C}$ & 18.3 & 8.3 & 0.7 & 14.6 & 58.1 & 18.4 \\
\hline 56.56 & $2.73 \mathrm{C}$ & 19.8 & 10.7 & 1.5 & 9.9 & 58.0 & 18.0 \\
\hline 56.71 & & 6.7 & 8.1 & 1.4 & 31.6 & 52.3 & 16.2 \\
\hline 56.86 & $3.07 \mathrm{C}$ & 7.8 & 15.0 & 0.6 & 13.8 & 62.8 & 14.7 \\
\hline 57.01 & $3.20 \mathrm{C}$ & 4.9 & 12.7 & 3.6 & 11.6 & 67.2 & 13.2 \\
\hline 57.16 & $3.45 \mathrm{C}$ & 8.2 & 10.5 & 5.1 & 15.2 & 61.0 & 14.3 \\
\hline 57.29 & & 2.7 & 7.8 & 12.2 & 17.2 & 60.1 & 11.2 \\
\hline 57.37 & & 3.6 & 7.7 & 13.1 & 14.2 & 61.3 & 11.4 \\
\hline 57.53 & $3.55 \mathrm{C}$ & 6.1 & 4.0 & 20.2 & 9.8 & 59.9 & 11.5 \\
\hline 57.63 & & 3.7 & 7.1 & 18.3 & 19.5 & 51.5 & 10.5 \\
\hline 57.78 & & 4.5 & 8.6 & 16.3 & 21.1 & 49.5 & 10.9 \\
\hline 57.91 & $3.75 \mathrm{U}$ & 2.7 & 6.5 & 0.4 & 22.1 & 68.4 & 14.4 \\
\hline 58.06 & $3.54 \mathrm{U}$ & 7.1 & 14.7 & 0.9 & 18.7 & 58.6 & 14.6 \\
\hline 58.21 & & 10.2 & 19.0 & 0.3 & 11.2 & 59.3 & 15.0 \\
\hline 58.35 & $2.80 \mathrm{C}$ & & & & & & \\
\hline 58.36 & & 6.9 & 12.9 & 1.4 & 23.8 & 55.0 & 15.0 \\
\hline 58.51 & $2.95 \mathrm{C}$ & 10.6 & 12.3 & 1.4 & 12.3 & 63.4 & 15.7 \\
\hline 58.66 & $3.41 \mathrm{C}$ & 6.8 & 12.8 & 4.1 & 19.6 & 56.7 & 14.0 \\
\hline 58.79 & & 6.2 & 13.1 & 9.9 & 9.1 & 61.7 & 12.2 \\
\hline 58.83 & $3.51 \mathrm{C}$ & & & & & & \\
\hline 58.87 & & 1.6 & 12.3 & 16.8 & 8.5 & 60.8 & 9.4 \\
\hline 59.03 & & 7.1 & 11.8 & 14.5 & 16.8 & 49.8 & 11.8 \\
\hline 59.09 & & 4.6 & 19.7 & 12.4 & 15.9 & 47.4 & 10.0 \\
\hline 59.19 & & 5.3 & 15.2 & 5.3 & 28.8 & 45.5 & 13.5 \\
\hline 59.28 & & 8.7 & 14.5 & 5.5 & 20.4 & 50.9 & 14.1 \\
\hline 59.41 & & 4.7 & 8.3 & 0.7 & 16.0 & 70.3 & 14.6 \\
\hline 59.50 & & 11.0 & 15.2 & 3.1 & 21.4 & 49.3 & 15.7 \\
\hline 59.56 & & 10.0 & 15.2 & 1.6 & 14.8 & 58.4 & 15.2 \\
\hline 59.71 & & 12.6 & 12.0 & 0.5 & 16.6 & 58.3 & 16.8 \\
\hline 59.86 & $2.10 \mathrm{C}$ & 15.3 & 8.4 & 1.1 & 10.2 & 65.0 & 17.4 \\
\hline 60.01 & & 15.6 & 12.0 & 2.7 & 18.3 & 51.5 & 17.2 \\
\hline 60.16 & & 4.4 & 11.3 & 11.6 & 20.4 & 52.4 & 11.5 \\
\hline 60.30 & & 2.8 & 15.3 & 17.9 & 15.3 & 48.6 & 9.1 \\
\hline 60.37 & & 1.1 & 14.2 & 19.5 & 23.8 & 41.4 & 8.0 \\
\hline 60.53 & $4.38 \mathrm{U}$ & 3.1 & 8.7 & 9.0 & 31.3 & 47.9 & 12.3 \\
\hline
\end{tabular}


Appendix C (continued).

\begin{tabular}{|c|c|c|c|c|c|c|c|}
\hline $\begin{array}{l}\text { Sub-bottom } \\
\text { depth } \\
\text { (m) }\end{array}$ & $\begin{array}{c}\delta^{18} \mathrm{O} \\
\left(\%_{0}\right)\end{array}$ & $\begin{array}{c}G . \\
\text { ruber } \\
(\%)\end{array}$ & $\begin{array}{c}\text { G. } \\
\text { bulloid. } \\
(\%)\end{array}$ & $\begin{array}{c}N . \\
\text { pachy. s. } \\
(\%)\end{array}$ & $\begin{array}{c}G . \\
\text { inflata } \\
(\%)\end{array}$ & $\begin{array}{l}\text { All } \\
\text { other } \\
(\%)\end{array}$ & $\begin{array}{l}\text { SSTw } \\
\left({ }^{\circ} \mathrm{C}\right)\end{array}$ \\
\hline 60.61 & $4.34 \mathrm{U}$ & 3.4 & 7.5 & 9.1 & 27.5 & 52.5 & 12.4 \\
\hline 60.78 & $4.11 \mathrm{U}$ & 2.1 & 12.5 & 3.9 & 23.8 & 57.7 & 12.3 \\
\hline 60.91 & $3.25 \mathrm{C}$ & 1.5 & 6.6 & 3.8 & 25.3 & 62.7 & 13.1 \\
\hline 61.06 & $2.92 \mathrm{C}$ & 8.2 & 12.0 & 1.3 & 25.1 & 53.5 & 15.8 \\
\hline 61.21 & $2.76 \mathrm{C}$ & 14.4 & 15.2 & 1.1 & 17.0 & 52.3 & 17.0 \\
\hline 61.36 & $2.67 \mathrm{C}$ & 18.8 & 11.4 & 0.0 & 14.8 & 55.0 & 18.6 \\
\hline 61.51 & & 12.2 & 9.3 & 1.4 & 22.9 & 54.1 & 17.2 \\
\hline 61.66 & $2.70 \mathrm{C}$ & 17.8 & 10.0 & 3.1 & 15.2 & 53.9 & 17.5 \\
\hline 61.81 & $2.74 \mathrm{C}$ & 12.5 & 10.3 & 4.5 & 18.6 & 54.2 & 16.0 \\
\hline 61.87 & & 2.7 & 9.9 & 16.0 & 19.4 & 52.0 & 10.1 \\
\hline 62.03 & $3.63 \mathrm{C}$ & 2.7 & 13.9 & 19.6 & 13.5 & 50.3 & 9.1 \\
\hline 62.11 & & 4.4 & 10.9 & 8.0 & 18.5 & 58.2 & 12.3 \\
\hline 62.19 & & 6.2 & 9.2 & 9.2 & 27.8 & 47.6 & 13.4 \\
\hline 62.28 & $3.80 \mathrm{C}$ & 5.2 & 8.7 & 11.7 & 23.6 & 50.7 & 12.2 \\
\hline 62.41 & & 2.0 & 8.2 & 14.1 & 19.7 & 55.9 & 10.4 \\
\hline 62.56 & & 4.1 & 11.1 & 19.0 & 22.2 & 43.7 & 10.0 \\
\hline 62.64 & & 8.7 & 13.0 & 9.4 & 18.7 & 50.2 & 13.3 \\
\hline 62.71 & & 12.2 & 14.1 & 8.0 & 19.2 & 46.6 & 14.8 \\
\hline 62.86 & $3.00 \mathrm{C}$ & 15.7 & 12.3 & 1.4 & 17.2 & 53.5 & 17.5 \\
\hline 63.01 & & 14.3 & 16.0 & 1.4 & 13.3 & 55.1 & 16.5 \\
\hline 63.16 & $3.02 \mathrm{C}$ & 18.0 & 16.1 & 0.9 & 20.2 & 44.7 & 18.6 \\
\hline 63.24 & & 16.6 & 14.5 & 1.1 & 23.1 & 44.8 & 18.6 \\
\hline 63.65 & & 3.1 & 11.3 & 6.8 & 31.3 & 47.6 & 12.7 \\
\hline 63.76 & & 2.8 & 8.8 & 7.7 & 30.3 & 50.4 & 12.5 \\
\hline 63.91 & & 1.0 & 9.9 & 13.4 & 36.7 & 39.0 & 10.3 \\
\hline 63.98 & & 1.7 & 7.9 & 12.7 & 41.1 & 36.6 & 11.6 \\
\hline 64.13 & $3.57 \mathrm{C}$ & 1.2 & 11.7 & 12.8 & 36.4 & 37.9 & 10.4 \\
\hline 64.21 & & 3.6 & 8.9 & 13.9 & 23.8 & 49.8 & 11.0 \\
\hline 64.38 & $3.44 \mathrm{C}$ & 4.1 & 10.8 & 13.2 & 24.9 & 47.1 & 11.2 \\
\hline 64.49 & $3.27 \mathrm{C}$ & 6.9 & 8.0 & 7.2 & 33.1 & 44.8 & 14.7 \\
\hline 64.66 & & 4.0 & 10.3 & 25.8 & 16.2 & 43.8 & 9.2 \\
\hline 64.73 & & 3.3 & 12.1 & 26.9 & 13.8 & 43.8 & 8.7 \\
\hline 64.81 & & 4.9 & 8.6 & 8.6 & 13.8 & 64.2 & 12.6 \\
\hline 64.89 & $3.70 \mathrm{U}$ & 5.6 & 5.6 & 4.0 & 24.6 & 60.1 & 14.7 \\
\hline 64.96 & $3.54 \mathrm{U}$ & 5.1 & 10.6 & 4.0 & 27.0 & 53.3 & 14.1 \\
\hline 65.13 & $3.44 \mathrm{U}$ & 14.6 & 15.7 & 3.0 & 20.6 & 46.1 & 16.9 \\
\hline 65.26 & $3.00 \mathrm{C}$ & 11.0 & 13.0 & 2.7 & 15.0 & 58.5 & 15.6 \\
\hline 65.26 & $3.37 \mathrm{U}$ & & & & & & \\
\hline 65.39 & $2.89 \mathrm{C}$ & & & & & & \\
\hline 65.41 & & 6.7 & 10.7 & 5.4 & 17.4 & 59.8 & 13.8 \\
\hline 65.48 & & 4.1 & 7.9 & 7.2 & 22.6 & 58.2 & 12.9 \\
\hline 65.63 & & 11.3 & 3.3 & 7.6 & 17.6 & 60.2 & 15.4 \\
\hline 65.71 & $4.16 \mathrm{U}$ & 5.4 & 8.3 & 8.0 & 17.4 & 60.9 & 13.0 \\
\hline 65.88 & $4.39 \mathrm{U}$ & 5.6 & 13.5 & 6.9 & 16.5 & 57.4 & 12.6 \\
\hline 66.01 & $4.34 \mathrm{U}$ & 6.6 & 11.0 & 6.6 & 12.1 & 63.6 & 13.3 \\
\hline 66.16 & $4.20 \mathrm{U}$ & 6.3 & 7.8 & 8.1 & 22.7 & 55.2 & 13.5 \\
\hline 66.29 & $4.03 \mathrm{U}$ & 4.8 & 7.5 & 9.2 & 29.0 & 49.5 & 13.0 \\
\hline 66.46 & $3.30 \mathrm{C}$ & 9.0 & 4.1 & 8.2 & 23.2 & 55.4 & 14.8 \\
\hline 66.61 & & 8.4 & 10.0 & 14.1 & 18.4 & 49.1 & 12.6 \\
\hline 66.76 & & 4.0 & 8.3 & 19.9 & 19.9 & 48.0 & 10.1 \\
\hline 66.81 & & 3.2 & 8.6 & 15.2 & 21.8 & 51.3 & 10.6 \\
\hline 66.91 & $3.94 \mathrm{U}$ & 4.0 & 9.4 & 5.4 & 16.4 & 64.8 & 13.0 \\
\hline 66.98 & & 4.4 & 8.2 & 5.1 & 20.8 & 61.4 & 13.5 \\
\hline 67.13 & $2.88 \mathrm{C}$ & 8.9 & 9.9 & 2.4 & 13.4 & 65.4 & 15.2 \\
\hline 67.23 & $2.69 \mathrm{C}$ & 12.3 & 11.0 & 1.7 & 11.3 & 63.7 & 16.3 \\
\hline 67.38 & & 13.3 & 10.7 & 2.6 & 16.5 & 57.0 & 16.6 \\
\hline 67.49 & $2.89 \mathrm{C}$ & 9.9 & 7.4 & 3.4 & 12.3 & 67.0 & 15.5 \\
\hline 67.66 & & 11.8 & 5.6 & 2.8 & 22.0 & 57.8 & 16.9 \\
\hline 67.79 & & 8.1 & 7.1 & 4.2 & 21.8 & 58.8 & 15.2 \\
\hline 67.96 & & 11.7 & 4.5 & 2.6 & 19.9 & 61.3 & 16.8 \\
\hline 68.11 & & 11.6 & 6.4 & 3.5 & 14.2 & 64.5 & 16.1 \\
\hline 68.26 & & 7.9 & 12.9 & 11.4 & 14.5 & 53.3 & 12.5 \\
\hline 68.31 & & 6.0 & 13.8 & 18.0 & 13.8 & 48.5 & 10.7 \\
\hline 68.41 & & 6.8 & 6.8 & 2.7 & 19.5 & 64.2 & 15.1 \\
\hline 68.47 & & 9.5 & 6.1 & 1.4 & 22.0 & 61.0 & 16.5 \\
\hline 68.63 & & 20.6 & 9.8 & 0.0 & 19.3 & 50.3 & 19.4 \\
\hline 68.71 & & 16.1 & 8.9 & 1.1 & 21.8 & 52.1 & 18.4 \\
\hline 68.88 & $3.15 \mathrm{C}$ & 14.5 & 8.0 & 2.1 & 17.3 & 58.1 & 17.3 \\
\hline 69.01 & & 9.2 & 7.5 & 2.9 & 11.3 & 69.1 & 15.4 \\
\hline 69.16 & $3.40 \mathrm{C}$ & 10.3 & 8.8 & 11.9 & 16.5 & 52.5 & 13.7 \\
\hline 69.31 & $3.74 \mathrm{C}$ & 9.5 & 8.9 & 3.9 & 20.3 & 57.4 & 15.5 \\
\hline 69.46 & $3.59 \mathrm{C}$ & 9.3 & 6.9 & 1.2 & 19.8 & 62.8 & 16.3 \\
\hline 69.61 & & 10.5 & 8.6 & 2.8 & 19.8 & 58.3 & 16.1 \\
\hline 69.76 & $3.68 \mathrm{C}$ & 10.5 & 6.8 & 8.4 & 24.0 & 50.3 & 15.0 \\
\hline 69.80 & & 8.8 & 4.6 & 17.3 & 24.0 & 45.3 & 12.5 \\
\hline 69.91 & $3.70 \mathrm{C}$ & 11.2 & 4.1 & 9.5 & 15.6 & 59.7 & 14.8 \\
\hline 70.13 & $3.15 \mathrm{C}$ & 14.2 & 8.2 & 0.7 & 17.5 & 59.4 & 17.6 \\
\hline 70.21 & & 13.7 & 9.7 & 4.3 & 21.3 & 51.0 & 16.7 \\
\hline 70.36 & $3.09 \mathrm{C}$ & & & & & & \\
\hline 70.38 & & 10.7 & 11.0 & 1.1 & 23.5 & 53.7 & 16.7 \\
\hline 70.51 & & 15.9 & 15.2 & 0.0 & 29.1 & 39.8 & 19.9 \\
\hline 70.66 & $2.86 \mathrm{C}$ & 17.0 & 19.5 & 0.4 & 23.8 & 39.4 & 19.2 \\
\hline
\end{tabular}

Appendix C (continued).

\begin{tabular}{|c|c|c|c|c|c|c|c|}
\hline $\begin{array}{l}\text { Sub-bottom } \\
\text { depth } \\
\text { (m) }\end{array}$ & $\begin{array}{l}\delta^{18} \mathrm{O} \\
\left(\%_{00}\right)\end{array}$ & $\begin{array}{l}G . \\
\text { ruber } \\
(\%)\end{array}$ & $\begin{array}{c}G . \\
\text { bulloid. } \\
(\%)\end{array}$ & $\begin{array}{c}N . \\
\text { pachy. s. } \\
(\%)\end{array}$ & $\begin{array}{c}G . \\
\text { inflata } \\
(\%)\end{array}$ & $\begin{array}{l}\text { All } \\
\text { other } \\
(\%)\end{array}$ & $\begin{array}{l}\text { SSTw } \\
\left({ }^{\circ} \mathrm{C}\right)\end{array}$ \\
\hline 70.81 & $3.10 \mathrm{C}$ & 13.7 & 12.3 & 1.4 & 21.6 & 51.0 & 17. \\
\hline 70.96 & & 15.4 & 4.1 & 1.5 & 22.9 & 56.0 & 18 \\
\hline 71.11 & $3.94 \mathrm{U}$ & 4.9 & 8.5 & 16.1 & 16.1 & 54.4 & 11. \\
\hline 71.26 & & 9.4 & 8.3 & 14.4 & 16.3 & 51.7 & 13.0 \\
\hline 71.39 & $3.50 \mathrm{C}$ & 1.0 & 14.5 & 17.7 & 13.8 & 53.1 & \\
\hline 71.48 & & 0.9 & 5.9 & 20.1 & 22.0 & 51.1 & 9.1 \\
\hline 71.63 & $3.10 \mathrm{C}$ & 6.2 & 9.3 & 14.1 & 20.3 & 50.2 & 11.9 \\
\hline 71.71 & & 18.3 & 7.3 & 6.4 & 16.2 & 51.7 & 16.8 \\
\hline 71.88 & $2.86 \mathrm{C}$ & 5.4 & 11.7 & 9.5 & 15.6 & 57.8 & 12.2 \\
\hline 71.88 & $3.58 \mathrm{U}$ & & & & & & \\
\hline 71.99 & $3.21 \mathrm{C}$ & 10.6 & 8.3 & 13.1 & 19.6 & 48.4 & 13. \\
\hline 71.99 & $3.60 \mathrm{U}$ & & & & & & \\
\hline 72.16 & $4.08 \mathrm{U}$ & 4.9 & 7.2 & 9.2 & 21.3 & 57.4 & 12. \\
\hline 72.31 & $3.05 \mathrm{C}$ & 4.8 & 7.9 & 12.0 & 23.4 & 51.9 & 12. \\
\hline 72.46 & $4.20 \mathrm{C}$ & 6.5 & 10.5 & 7.8 & 13.1 & 62.1 & 13. \\
\hline 72.61 & & 10.6 & 11.0 & 11.0 & 13.5 & 53.9 & 13 \\
\hline 72.65 & & 8.7 & 11.6 & 9.4 & 14.2 & 56.1 & 13 \\
\hline 73.21 & & 20.5 & 10.1 & 1.4 & 16.9 & 51.1 & 18. \\
\hline 73.36 & $2.73 \mathrm{C}$ & 25.2 & 8.3 & 1.1 & 16.5 & 48.9 & 19 \\
\hline 73.51 & & 24.1 & 13.3 & 1.8 & 20.8 & 40.1 & 19. \\
\hline 73.73 & $2.90 \mathrm{C}$ & 23.4 & 6.1 & 2.4 & 14.9 & 53.2 & 18. \\
\hline 73.81 & $2.91 \mathrm{C}$ & 20.9 & 7.4 & 1.2 & 20.7 & 49.8 & 19 \\
\hline 73.98 & $3.12 \mathrm{C}$ & 18.7 & 9.0 & 9.4 & 24.5 & 38.4 & 16 \\
\hline 74.11 & & 8.9 & 10.9 & 13.7 & 7.2 & 59.4 & 12 \\
\hline 74.26 & $3.35 \mathrm{C}$ & 6.2 & 14.7 & 16.8 & 12.4 & 49.9 & 10 \\
\hline 74.41 & & 4.1 & 9.5 & 14.6 & 19.0 & 52.9 & 11. \\
\hline 74.56 & & 5.5 & 8.2 & 14.1 & 12.0 & 60.1 & 11 \\
\hline 74.71 & $4.03 \mathrm{U}$ & 3.6 & 11.1 & 3.0 & 23.0 & 59.3 & 13 \\
\hline 74.86 & $3.89 \mathrm{U}$ & 5.1 & 9.5 & 1.2 & 23.6 & 60.7 & 14 \\
\hline 75.01 & $3.64 \mathrm{U}$ & 7.5 & 9.6 & 1.8 & 30.5 & 50.6 & 16. \\
\hline 75.23 & $2.68 \mathrm{C}$ & 9.4 & 6.2 & 0.7 & 25.1 & 58.6 & 17. \\
\hline 75.31 & & 11.3 & 7.0 & 1.8 & 18.3 & 61.6 & 16.6 \\
\hline 75.48 & & 11.1 & 6.9 & 0.5 & 20.9 & 60.7 & 17. \\
\hline 75.61 & $2.95 \mathrm{C}$ & 12.0 & 7.5 & 1.2 & 18.4 & 60.8 & 17 \\
\hline 75.76 & & 11.4 & 6.1 & 0.7 & 18.6 & 63.2 & 17.1 \\
\hline 75.91 & & 9.3 & 11.0 & 1.7 & 19.6 & 58.4 & 15.7 \\
\hline 76.06 & $2.81 \mathrm{C}$ & 8.7 & 9.1 & 0.7 & 18.9 & 62.6 & 16 \\
\hline 76.21 & (1) & 4.0 & 8.3 & 1.9 & 11.3 & 74.5 & 14.0 \\
\hline 76.28 & & 2.6 & 4.5 & 1.4 & 18.6 & 72.8 & 14.2 \\
\hline 76.36 & $4.33 \mathrm{U}$ & 2.9 & 12.9 & 1.9 & 22.2 & 60.1 & 13.1 \\
\hline 76.51 & & 6.3 & 7.0 & 1.4 & 16.9 & 68.3 & 15.1 \\
\hline 76.73 & $3.01 \mathrm{C}$ & 6.3 & 10.7 & 1.7 & 24.0 & 57.3 & 15.0 \\
\hline 76.73 & $3.55 \mathrm{U}$ & & & & & & \\
\hline 76.81 & $3.66 \mathrm{U}$ & 8.7 & 16.4 & 1.7 & 23.2 & 50.0 & 15.3 \\
\hline 76.98 & $3.62 \mathrm{U}$ & 7.4 & 9.1 & 1.8 & 18.5 & 63.2 & 15.2 \\
\hline 76.98 & $3.01 \mathrm{C}$ & & & & & & \\
\hline 77.11 & $2.82 \mathrm{C}$ & 13.8 & 9.4 & 2.0 & 16.2 & 58.6 & 17.0 \\
\hline 77.26 & $2.94 \mathrm{C}$ & 9.9 & 7.3 & 0.0 & 22.1 & 60.7 & 17 \\
\hline 77.43 & & 5.4 & 12.7 & 1.0 & 20.7 & 60.2 & 14 \\
\hline 77.56 & & 6.5 & 10.7 & 1.8 & 17.3 & 63.7 & \\
\hline 77.73 & $3.56 \mathrm{U}$ & 13.9 & 14.7 & 1.1 & 15.4 & 54.9 & 16 \\
\hline 77.86 & $2.83 \mathrm{C}$ & 10.0 & 12.9 & 0.9 & 10.0 & 66.2 & 15.5 \\
\hline 78.01 & & 18.8 & 11.1 & 2.3 & 10.0 & 57.9 & 17.6 \\
\hline 78.23 & & 16.7 & 7.6 & 2.3 & 16.7 & 56.7 & 17.7 \\
\hline 78.31 & & 15.4 & 12.9 & 1.7 & 11.2 & 58.7 & 16 \\
\hline 78.48 & & 15.7 & 9.5 & 0.9 & 8.0 & 66.0 & 17 \\
\hline 78.61 & & 18.3 & 6.9 & 1.0 & 2.4 & 71.4 & 18 \\
\hline 78.76 & & 16.1 & 6.8 & 1.3 & 1.6 & 74.3 & 17 \\
\hline 78.91 & & 25.6 & 6.9 & 2.3 & 8.4 & 56.8 & 18.6 \\
\hline 79.06 & & 16.7 & 8.8 & 2.6 & 11.8 & 60.1 & 17 \\
\hline 79.21 & & 11.4 & 9.5 & 3.2 & 24.4 & 51.6 & 16 \\
\hline 79.36 & & 10.5 & 14.5 & 0.7 & 22.1 & 52.2 & 16 \\
\hline 79.51 & & 5.3 & 7.5 & 1.7 & 21.7 & 63.8 & 14 \\
\hline 79.73 & & 7.0 & 10.5 & 0.7 & 22.0 & 59.8 & 15 \\
\hline 79.81 & & 5.6 & 10.2 & 3.6 & 21.9 & 58.7 & 14 \\
\hline 79.98 & & 5.1 & 11.0 & 3.3 & 22.1 & 58.5 & 13 \\
\hline 80.26 & & 6.5 & 7.7 & 1.5 & 18.9 & 65.3 & 15 \\
\hline 80.41 & & 3.1 & 7.9 & 2.4 & 22.8 & 63.8 & 13 \\
\hline 80.56 & & 1.4 & 13.6 & 2.9 & 21.1 & 61.1 & 12 \\
\hline 80.61 & & 2.5 & 10.6 & 2.7 & 26.0 & 58.2 & 13 \\
\hline 80.71 & $3.11 \mathrm{C}$ & 4.5 & 17.4 & 3.0 & 24.2 & 50.9 & 13 \\
\hline 80.71 & $3.80 \mathrm{U}$ & & & & & & \\
\hline 80.86 & & 9.6 & 11.5 & 2.1 & 21.7 & 55.0 & 15 \\
\hline 80.99 & & 5.5 & 11.5 & 2.6 & 13.2 & 67.2 & 13 \\
\hline 81.23 & & 11.2 & 12.2 & 1.6 & 11.5 & 63.5 & 15 \\
\hline 81.31 & $2.82 \mathrm{C}$ & 10.1 & 10.7 & 2.9 & 15.9 & 60.3 & 15 \\
\hline 81.48 & & 9.9 & 14.4 & 4.9 & 17.1 & 53.6 & 14 \\
\hline 81.59 & & 6.0 & 18.0 & 3.2 & 13.8 & 59.0 & 12 \\
\hline 81.76 & $3.40 \mathrm{C}$ & 5.1 & 15.9 & 0.7 & I4.1 & 64.1 & 13 \\
\hline 81.91 & & 8.7 & 12.1 & 1.7 & 12.1 & 65.4 & 15 \\
\hline 82.06 & & 8.6 & 9.8 & 2.0 & 7.8 & 71.8 & 15 \\
\hline 82.21 & & 3.0 & 7.0 & 1.8 & 13.7 & 74.4 & 13.9 \\
\hline
\end{tabular}


W. F. RUDDIMAN, A. MCINTYRE, AND M. RAYMO

Appendix C (continued).

\begin{tabular}{cccccccc}
\hline $\begin{array}{c}\text { Sub-bottom } \\
\text { depth } \\
(\mathrm{m})\end{array}$ & $\begin{array}{c}\delta^{18} \mathrm{O} \\
(\% 0)\end{array}$ & $\begin{array}{c}\text { G. } \\
\text { ruber } \\
(\%)\end{array}$ & $\begin{array}{c}\text { G. } \\
\text { bulloid. } \\
(\%)\end{array}$ & $\begin{array}{c}N . \\
\text { pachy. s. } \\
(\%)\end{array}$ & $\begin{array}{c}G . \\
\text { inflata } \\
(\%)\end{array}$ & $\begin{array}{c}\text { All } \\
\text { other } \\
(\%)\end{array}$ & $\begin{array}{c}\text { SSTw } \\
\left({ }^{\circ} \mathrm{C}\right)\end{array}$ \\
\hline 82.36 & & 7.8 & 10.6 & 1.4 & 12.8 & 67.4 & 15.0 \\
82.51 & $3.31 \mathrm{C}$ & 3.0 & 10.7 & 1.7 & 13.7 & 71.0 & 13.3 \\
\hline
\end{tabular}

Note: Oxygen isotope values are unadjusted and are relative to PDB standard. A " $\mathrm{U}$ " indicates Uvigerina, and a " $\mathrm{C}$ " indicates Cibicides. The planktonic foraminifer species name abbreviations stand for: $G$. ruber-Globigerinoides ruber white; G. bulloid.-Globigerina bulloides; N. pachy. s.-Neogloboquadrina pachyderma sinistral; $G$. inflata - Globorotalia inflata; All other-all other species combined. SSTw stands for estimated winter sea-surface temperature. 